\title{
The Glycocalyx and Its Role in Vascular Physiology and Vascular Related Diseases
}

\author{
Sheldon Weinbaum, Limary M. Cancel (D, Bingmei M. Fu, and John M. Tarbell \\ Biomedical Engineering Department, The City College of New York, New York, NY, USA \\ (Received 30 June 2020; accepted 2 September 2020; published online 21 September 2020)
}

Associate Editor Hanjoong Jo oversaw the review of this article.

\begin{abstract}
Purpose-In 2007 the two senior authors wrote a review on the structure and function of the endothelial glycocalyx layer (Weinbaum in Annu Rev Biomed Eng 9:121-167, 2007). Since then there has been an explosion of interest in this hydrated gel-like structure that coats the luminal surface of endothelial cells that line our vasculature due to its important functions in (A) basic vascular physiology and (B) vascular related diseases. This review will highlight the major advances that have occurred since our 2007 paper.

Methods-A literature search mainly focusing on the role of the glycocalyx in the two major areas described above was performed using electronic databases.

Results - In part (A) of this review, the new formulation of the century old Starling principle, now referred to as the Michel-Weinbaum glycoclayx model or revised Starling hypothesis, is described including new subtleties and physiological ramifications. New insights into mechanotransduction and release of nitric oxide due to fluid shear stress sensed by the glycocalyx are elaborated. Major advances in understanding the organization and function of glycocalyx components, and new techniques for measuring both its thickness and spatio-chemical organization based on super resolution, stochastic optical reconstruction microscopy (STORM) are presented. As discussed in part (B) of this review, it is now recognized that artery wall stiffness associated with hypertension and aging induces glycocalyx degradation, endothelial dysfunction and vascular disease. In addition to atherosclerosis and cardiovascular diseases, the glycocalyx plays an important role in lifestyle related diseases (e.g., diabetes) and cancer. Infectious diseases including sepsis, Dengue, Zika and Corona viruses, and malaria also involve the glycocalyx. Because of increasing recognition of the role of the glycocalyx in a wide range of diseases, there has been a vigorous search for methods to protect the
\end{abstract}

Address correspondence to Limary M. Cancel, Biomedical Engineering Department, The City College of New York, New York, NY, USA. Electronic mail: limarycancel@yahoo.com glycocalyx from degradation or to enhance its synthesis in disease environments.

Conclusion - As we have seen in this review, many important developments in our basic understanding of GCX structure, function and role in diseases have been described since the 2007 paper. The future is wide open for continued GCX research.

Keywords-Endothelial glycocalyx, Mechanotransduction, Revised Starling principle, Michel-Weinbaum model, Cancer cell glycocalyx.

\section{INTRODUCTION}

In 2007 the two senior authors and ER Damiano wrote a highly cited extensive review ${ }^{220}$ summarizing what was known at the time of the structure and function of the endothelial glycocalyx (GCX), the hydrated gel-like structure that coats the luminal surface of the endothelial cells (EC) that line our vasculature. This surface matrix is comprised of sulfated proteoglycans - the transmembrane syndecans and the membrane bound glypicans, with their covalently bound glycosaminoglycans (GAGs) - heparan sulfate (HS) and chondroitin sulfate (CS) and the non-sulfated GAG hyaluronic acid (HA), as well as glycoproteins and plasma proteins (see Fig. 1). In the past decade there has been an explosion of interest in this hydrated gel-like structure due to its important functions in (A) basic vascular physiology and (B) vascular related diseases. In Part A we describe how the GCX has led to a new formulation of the century old Starling principle ${ }^{195}$ now referred to as the Michel-Weinbaum glycoclayx model $^{134,219}$ or the revised Starling hypothesis for microvascular fluid exchange. There have been new insights into mechanotransduction and the release of nitric oxide (NO) due to fluid shear stress (FSS), ${ }^{50,60,231}$ major advances in our understanding of 


\section{Lumen}

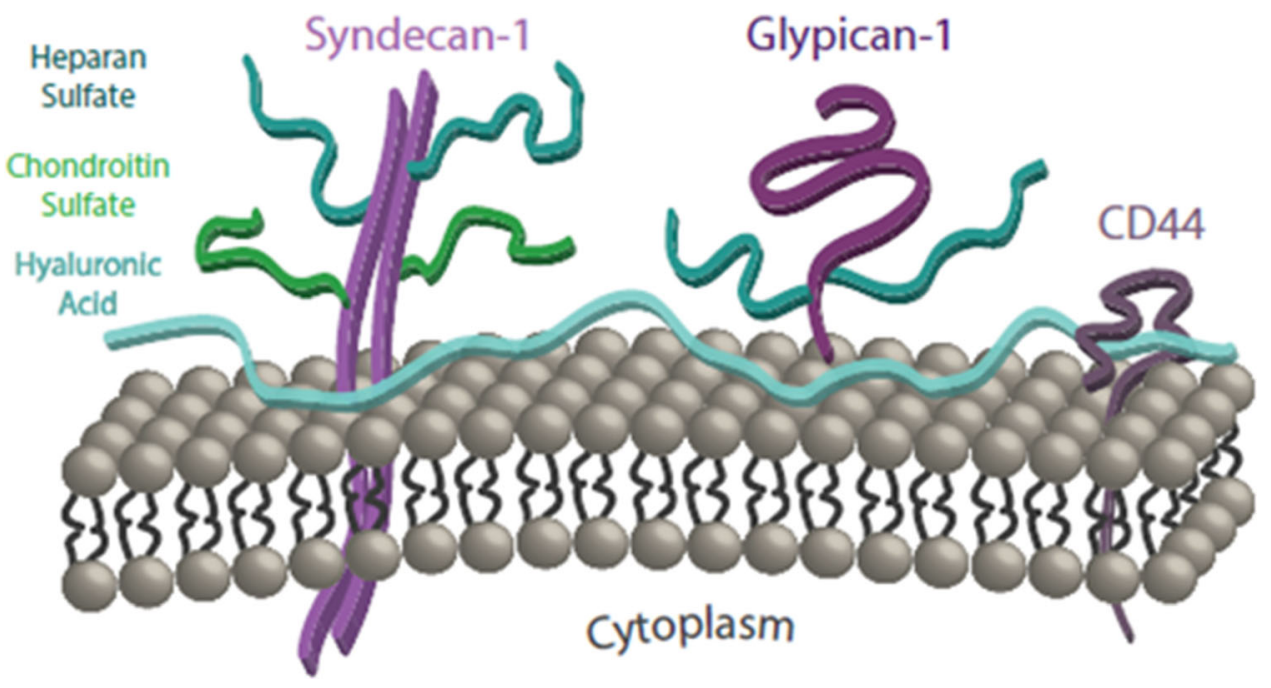

FIGURE 1. Simplified schematic of the glycocalyx: cell-surface core proteins and their associated GAGs. Reprinted from Ref. 12.

the structure and function of its various components, ${ }^{159,236}$ and new techniques for measuring both its thickness and structural organization based on confo$\mathrm{cal}^{232}$ rapid freeze substitution ${ }^{51}$ and super resolution stochastic optical reconstruction microscopy (STORM). ${ }^{57}$

In Part B we emphasize that it is now recognized that artery wall stiffness associated with hypertension and aging induces GCX degradation, endothelial dysfunction and vascular disease. ${ }^{120}$ In addition to atherosclerosis and cardiovascular diseases, the GCX plays an important role in lifestyle related diseases (e.g., diabetes) and cancer. ${ }^{200}$ Infectious diseases including sepsis, Dengue, Zika and Corona viruses, and malaria also involve the GCX. ${ }^{85,136,167}$ Because of increasing recognition of the role of the GCX in a wide range of diseases, there has been a vigorous search for methods to protect the GCX from degradation or to enhance its synthesis in disease environments. ${ }^{200}$

This review will highlight the major advances outlined above that have occurred since the 2007 paper.

\section{PART A: THE ROLE OF THE GLYCOCALYX IN VASCULAR PHYSIOLOGY}

\section{The Revised Starling Principle: Michel-Weinbaum Model for Microvascular Fluid Exchange}

It is relatively rare that a widely accepted textbook description of a basic microcirculatory phenomenon should be challenged after nearly a century of use. However, this is indeed what happened with the classical Starling hypothesis. While the basic ideas for the revised Starling hypothesis were introduced by Michel $^{134}$ and Weinbaum ${ }^{219}$ more than 20 years ago and the experimental verification of the MichelWeinbaum model confirmed in mesentery venules in frog by $\mathrm{Hu}$ et $a l .{ }^{77}$ and rat by Adamson et al. ${ }^{1}$ more than a decade ago, the widespread acceptance of the revised hypothesis had not occurred when our 2007 review paper on the GCX was published. Two new contributions greatly changed this. In 2010 Levick and Michel ${ }^{108}$ wrote a Spotlight Review in Cardiovascular Research which emphasized the clinical importance of the revised principle and more recently Tom Woodcock, an English clinician, prepared a 27 min video emphasizing the clinical applications of the revised hypothesis. This video is the lead entry if you do a Google search for the Michel-Weinbaum model which currently has $>100,000$ listings.

In the present review, after a brief summary of the basic differences between the classic and revised Starling hypothesis, we will highlight the fundamental differences between one and two-dimensional models and the role that trapped microdomains within the intercellular cleft and at the cleft exit play in the time dependent response that leads to steady state behavior.

\section{Classic Starling principle}

From classic thermodynamic principles one can write the volume filtration rate per unit area of endothelial surface or fluid flux $J_{\mathrm{v}} / A$ as a balance between two forces, the difference in hydrostatic pressures across a membrane $P_{\mathrm{L}}-P_{\mathrm{i}}$ and the difference in oncotic pressures $\pi_{\mathrm{p}}-\pi_{\mathrm{i}}$ where the subscripts $\mathrm{L}$ and $\mathrm{i}$ refer to the lumen of the microvessel and the 
interstitial fluid (ISF) in the tissue and $\pi_{\mathrm{P}}$ is the oncotic pressure of plasma. If this was a simple membrane with pores one could write the expression for $J_{\mathrm{v}} / A$ as

$$
\frac{J_{\mathrm{v}}}{A}=L_{\mathrm{p}}\left[P_{\mathrm{L}}-P_{\mathrm{i}}-\sigma\left(\pi_{\mathrm{p}}-\pi_{\mathrm{i}}\right)\right],
$$

where $L_{\mathrm{p}}$ and $\sigma$ are the filtration coefficient (or hydraulic conductivity) and osmotic reflection coefficient respectively. The value for $\sigma$ varies between 0 and 1 where 0 would be for a solute much smaller than the membrane pores and 1 for a plasma protein that was too large to pass through the pore. Starling realized that the plasma proteins in the blood and, in particular the most abundant plasma protein, albumin, would be greatly hindered and could have a value of $\sigma$ that would be close to one creating a colloidal oncotic pressure (COP) for fluid absorption.

For many years it was widely believed that the pores in the EC monolayer were the intercellular clefts between adjacent ECs and the constricting pores were either a matrix in the cleft or associated with the junction strands between the ECs which served as the molecular filter for the plasma proteins. The other basic assumption in the original hypothesis was that the COP in the plasma, which could be measured, was typically $25-28 \mathrm{mmHg}$ and the interstitial COP was close to zero, though the techniques for measuring the latter were not developed until the 1980s. Using classic micropipette measuring techniques developed by Landis $^{101}$ and Pappenheimer and Soto-Rivera, ${ }^{161}$ one observed that at heart level the typical hydrostatic pressure on the arterial side of capillaries was $35 \mathrm{mmHg}$ whereas on the venous side a typical value was $15 \mathrm{mmHg}$. Thus, when the capillary pressure $P_{\mathrm{L}}$ was $>25 \mathrm{mmHg}$, water would leave the capillary. When $P_{\mathrm{L}}$ was $<25 \mathrm{mmHg}$ water would be reabsorbed. Thus, several generations of medical students were taught using Eq. (1) that the plasma that filtered out of our capillaries when $P_{\mathrm{L}}$ was $>\pi_{\mathrm{P}}$, and was reabsorbed on the venous side because the COP of our plasma proteins $\pi_{\mathrm{P}}$ was $>P_{\mathrm{L}}$. This was often referred to as the non-steady state absorption rule applied at heart level.

With the advent of reliable techniques for measuring the COP of the interstitial space it was discovered that $\pi_{\mathrm{i}}$ was not close to zero but typically $40 \%$ of $\pi_{\mathrm{P}}$. This extraordinary observation was first reported in 1991 by Levick ${ }^{107}$ who showed that in the 14 tissues, including muscle, skin and lung, where $\pi_{\mathrm{i}}$ had been measured there was no venous absorption. The only exceptions were the renal cortex, where fluid is continuously taken up by the peritubular capillaries, and the ascending vasa recta of the intestinal mucosa where protein free fluid is continuously secreted by neighboring epithelia, see Levick and Michel. ${ }^{108}$

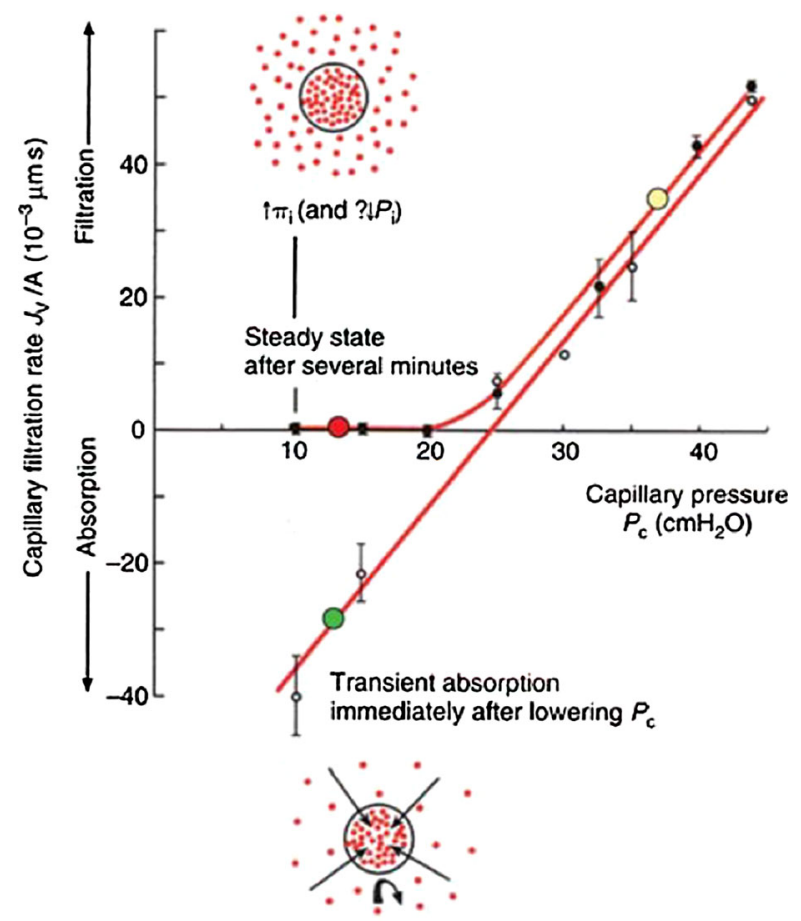

FIGURE 2. Water flux $J_{v} / A$ of frog mesentery capillary as a function of capillary pressure in single perfused frog mesentery capillary. Note the transient state in which measurements are performed in the first $15 \mathrm{~s}$ after the perfusion pressure is fixed fall along a straight line in which absorption does occur for pressures below $25 \mathrm{~cm} \mathrm{H}_{2} \mathrm{O}$, whereas steady-state measurements performed 2-5 min after perfusion pressure is fixed show no absorption, but a low level of filtration at low capillary pressures. Reprinted from Ref. 108.

\section{Revised Starling Principle}

In 1996 Levick and Michel organized a centennial meeting in London to celebrate the 100th anniversary of Starling's classic 1896 paper. The highlight of this meeting was Levick's presentation summarizing the results of his 1991 paper, ${ }^{107}$ Fig. $2 b$ in Ref. 108. showing the imbalance of the four classical Starling pressures when all four terms are measured in the same tissue and species. On the last day of the meeting Levick, Michel, Curry and Weinbaum met to try and understand how it was possible that the predictions of Eq. (1), the classic Starling force balance, could be so far off in nearly all tissues where these forces had been measured. This situation was further complicated by Michel and Phillips now classic experiment ${ }^{135}$ shown in Fig. 2 using the Landis technique applied to single perfused frog mesentery capillaries where the fluid exchange is measured immediately after raising or lowering the capillary pressure $P_{\mathrm{c}}$ (transient state) and after $P_{\mathrm{c}}$ has been held constant for 2-5 min (steady state). The transient state clearly satisfied the classic Starling force balance when $P_{\mathrm{c}}$ was less than $\pi_{\mathrm{L}}$ 


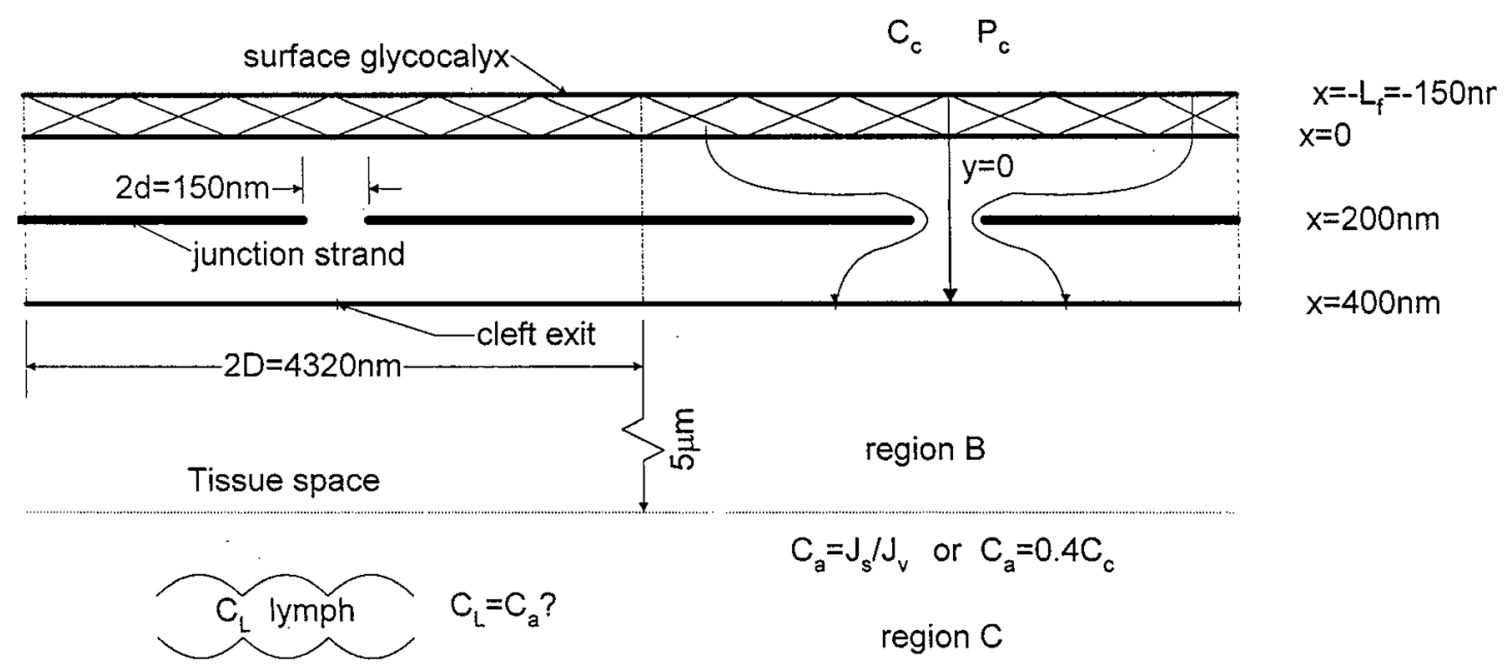

FIGURE 3. Schematic of idealized mathematical model showing surface matrix layer, cleft with junction strand, and tissue regions $B$ and $C$ describing mixing at cleft exit. Dimensions shown are typical of frog mesentery capillary. Reprinted from Ref. 219.

whereas in the steady state $J_{\mathrm{v}} / A$ always returned to a very low rate of filtration and the curve had the characteristic shape of a hockey stick. The latter behavior is described in detail in a recent review by Curry. ${ }^{37}$

The shape of the steady state curve in Fig. 2 can be predicted from a standard one-dimensional convection-diffusion equation in which one requires that in the far field at steady state the tissue concentration $C_{\mathrm{i}}$ is equal to the ratio of the solute influx from all sources $J_{\mathrm{s}}$ to the total water flux $J_{\mathrm{v}}$ or $C_{\mathrm{i}}=J_{\mathrm{s}} / J_{\mathrm{v}}$. The solute crossing the endothelium can come not only from fluid flow though the intercellular clefts but also from vesicular transport or back loading and $C_{\mathrm{i}}$ varies inversely with $J_{\mathrm{v}}$. Michel ${ }^{133}$ realized that as $J_{\mathrm{v}}$ decreased for the steady state curve in Fig. 2, the concentration in the tissue would have to approach the concentration in the plasma and reabsorption would vanish. The relation between $J_{\mathrm{v}}$ and $P_{\mathrm{c}}$ is non-linear since $\mathrm{J}_{\mathrm{v}}$ depends on the extracapillary protein concentration. The remarkable feature of Michel's transport equation is that it could reproduce the shape of the steady state curve in Fig. 2, with its hockey stick like bend, by varying the value of a single parameter, the permeability coefficient $P_{\mathrm{A}}$ for albumin in the expression for the Peclet number $\left(J_{\mathrm{v}} / A\right)(1-\sigma) / P_{\mathrm{A}}$ without ever including the cleft or its tight junction strand or the mixing of the ISF at the cleft exit. This unusual behavior will be explained with the aid of the twodimensional model in Fig. 3 where these features are included.

What Michel and Weinbaum independently realized after the 1996 meeting was that the molecular sieve for plasma proteins, and in particular albumin, did not lie within structures within the cleft itself but the GCX at the surface of the endothelium which clearly covered the cleft entrance. This new view was presented by Michel in his 1997 review paper summarizing the results of the Starling centennial meeting ${ }^{134}$ and Weinbaum in his 1997 Whitaker Distinguished Lecture at the annual Meeting of BMES in La Jolla. ${ }^{219}$ Michel intuitively realized that the protein concentration gradients within the cleft would be small if the GCX was a formidable barrier to diffusion and his conceptual model also included alternate non-convective pathways for larger molecules, such as vesicular transport which bypassed the clefts entirely. Weinbaum, on the other hand, shared Michel's view of the GCX, as the primary molecular barrier, but was also concerned about the role of the tight junction strands, since if the breaks in the strands were small and widely spaced the convective transport of proteins through the breaks could greatly impede back diffusion of proteins from the tissue. If this happened the concentration of the proteins behind the GCX could differ greatly from the ISF. Such non-uniformity in behavior would need to be described by a two-dimensional transport equation in the cleft.

\section{Weinbaum's 2-D Transport Model}

A sketch of the two-dimensional transport model used in Weinbaum ${ }^{219}$ is shown in Fig. 3. Instead of Eq. (1) one applies the Starling force balance just across the GCX

$$
\frac{J_{\mathrm{v}}}{A}=L_{\mathrm{p}}\left[P_{\mathrm{L}}-P_{\mathrm{G}}(y)-\sigma\left(\pi_{\mathrm{p}}-\pi_{\mathrm{G}}(y)\right)\right] .
$$

Here the subscript $\mathrm{G}$ refers to the protein concentration just behind the GCX ( $x=0$ in Fig. 3$)$ and one allows both the pressure and concentration to vary with lateral position y behind the GCX. As shown in 


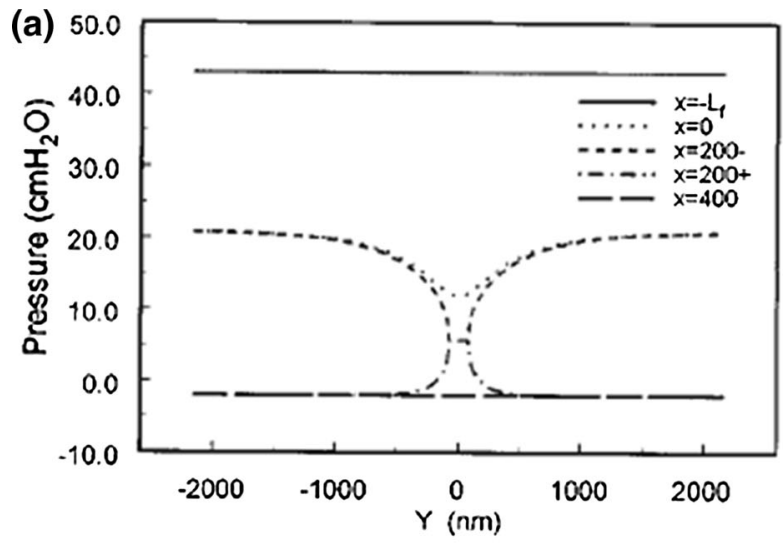

(b)

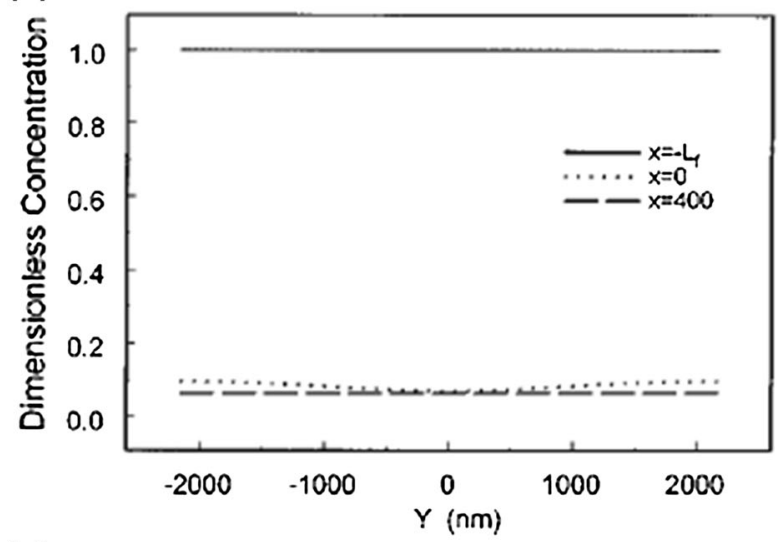

(c)

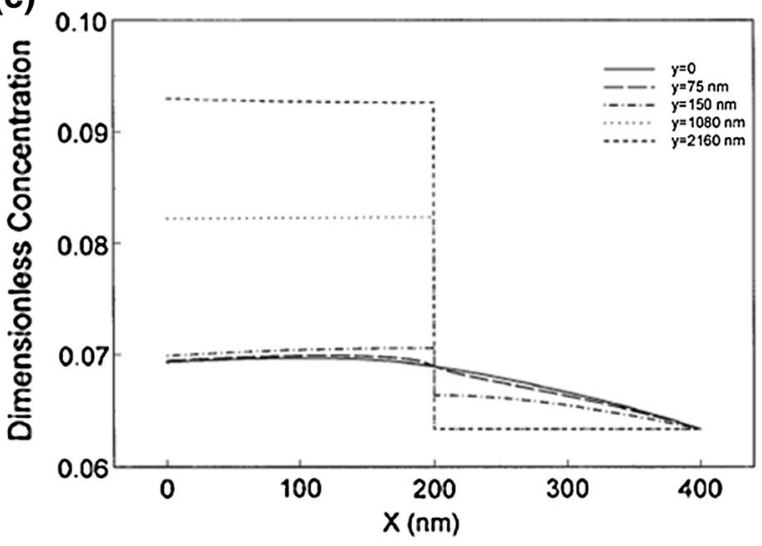

FIGURE 4. Predictions of theoretical model for $P_{\mathrm{c}}=43 \mathrm{~cm}$ $\mathrm{H}_{2} \mathrm{O}$ for pressure (a), protein concentration profiles at various locations (b, c) in cleft identified in Fig. 3. (a) and (b) reprinted from Ref. 219; (c) reprinted from Ref. 78.

Fig. 3 the breaks are periodically arranged along the length of the junction strand at a distance $2 \mathrm{D}=4320 \mathrm{~nm}$, where $y=0$ is at the center of each break whose width $2 d$ is $150 \mathrm{~nm}$. These dimensions are typical for frog mesentery venules. The model has four regions, (1) a GCX of thickness $150 \mathrm{~nm}$, (2) the cleft itself whose depth is $400 \mathrm{~nm}$ with a junction strand at $x=200 \mathrm{~nm}$, (3) a mixing region $\mathrm{B}$ of radius $5 \mu \mathrm{m}$, the half distance for the separation of the clefts, and (4) a

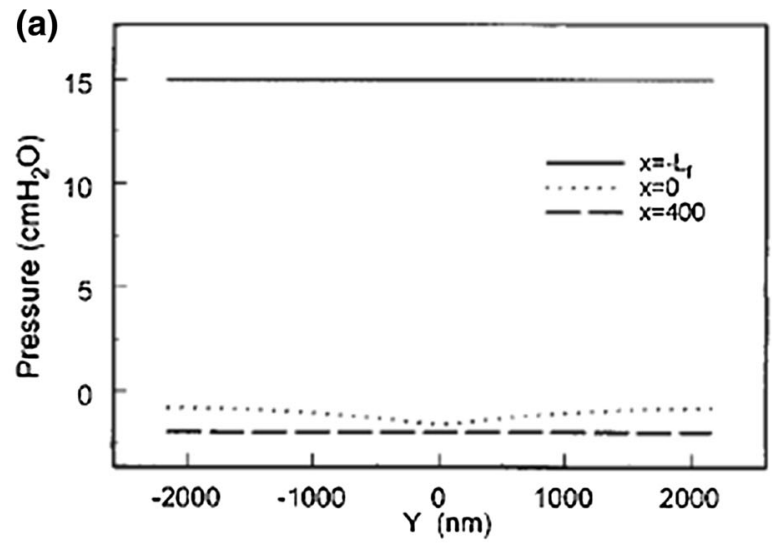

(b)

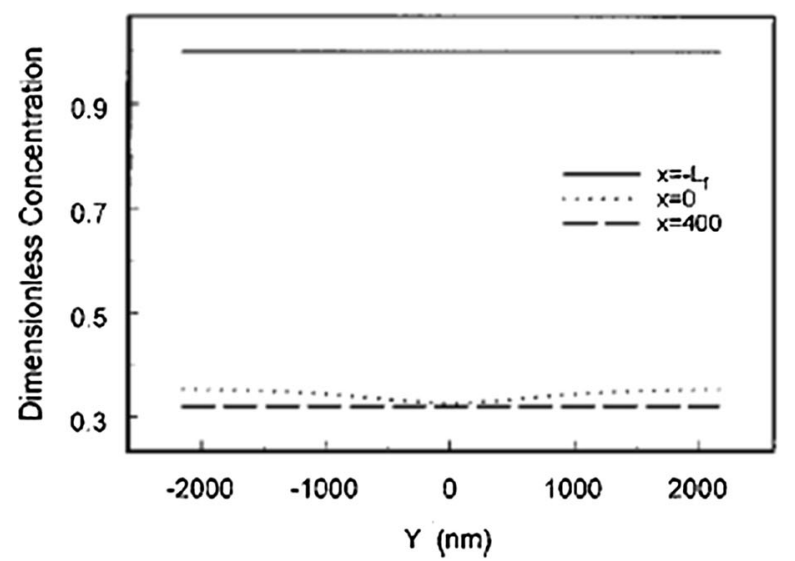

(c)

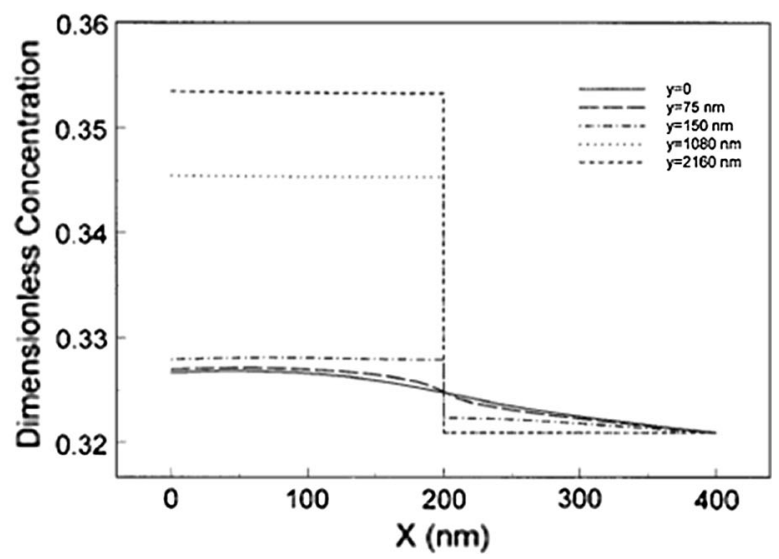

FIGURE 5. Predictions of theoretical model for $P_{\mathrm{c}}=15 \mathrm{~cm}$ $\mathrm{H}_{2} \mathrm{O}$ for pressure (a), protein concentration profiles at various locations (b, c) in cleft identified in Fig. 3. (a) and (b) Reprinted from Ref. 219; (c) reprinted from Ref. 78.

mixing region $\mathrm{C}$ of width $100 \mu \mathrm{m}$, the half distance between adjacent venules. The concentration in region C is set at the value $C_{\mathrm{i}}=C_{\mathrm{A}}=J_{\mathrm{s}} / J_{\mathrm{v}}$ as proposed in Michel or $C_{\mathrm{i}}=0.4 C_{\mathrm{L}}$ as observed in Levick. ${ }^{107} \mathrm{~A}$ unique feature of the model is that the flow in the cleft is treated as a 2-D Hele-Shaw flow so that the detailed pressure distribution and velocity field within the cleft 
can be examined and this 2-D velocity field used in the transport equation for the solute concentration.

We first examine the results of this 2-D model for Michel and Phillips steady state experiments in Fig. 2 for two cases, $P_{\mathrm{c}}=43 \mathrm{~cm} \mathrm{H}_{2} \mathrm{O}$, a high filtration state, and $P_{\mathrm{c}}=15 \mathrm{~cm} \mathrm{H} \mathrm{H}_{2} \mathrm{O}$, a value significantly below plasma oncotic pressure, Figs. 4 and 5 respectively. There is a small negative swelling pressure in the tissue of $-2 \mathrm{mmHg} .{ }^{68}$ In Fig. 4 a we see that at the high pressure, $43 \mathrm{~cm} \mathrm{H}_{2} \mathrm{O}$, nearly half of the pressure drop occurs across the GCX and there is a significant additional drop in pressure as the water is funneled into the break in the junction strand. The pressure drop across the junction strand at $x=200 \mathrm{~nm}$ is nearly the same as the pressure drop across the GCX. The breadth of the spread in pressure on the GCX side of the break is wider than on the tissue side leading to a narrower water jet on the exit side than the entrance side (velocity profiles not shown). More striking in Fig. $4 \mathrm{~b}$ is the fact that the concentration profile behind the GCX at $x=0$ is nearly identical to that at the cleft exit. The albumin diffusion coefficient in the cleft is assumed to be that of free diffusion $D_{\infty}$ whereas that in the GCX $D_{\mathrm{G}}$ is 1000 times smaller. This value of $D_{\mathrm{G}}$ provides for a very close fit of the bend in the curve shown in Fig. 2 for Michel and Phillips steady state experiments. This observation, that the concentration in the cleft is nearly uniform, is central to Michel's ${ }^{133}$ simplified one-dimensional model. Note also that in this high filtration limit $\mathrm{C}$ at the cleft exit has the limiting value $(1-\sigma) C_{\mathrm{L}}$ for pure filtration across the GCX.

The near uniformity in concentration at the cleft exit in Fig. 4b may seem surprising since there is a spike in the velocity profiles (not shown to save space) downstream of the break in the junction strand. The detailed concentration profiles at different y locations show why this is the case. Along $y=0$, the cleft centerline, the concentration does drop continuously along the cleft centerline from a value 0.07 to near 0.06 close to the convection limit since $\sigma=0.94$. One notes that there is a small but not insignificant drop in concentration, roughly $3 \%$, across the junction strand at larger values of y due to the diffusional spreading in the y direction. Convection is not important since there is hardly any fluid motion in the y direction except in the immediate vicinity of the junction strand breaks. Michel's ${ }^{133}$ model does not consider any mixing processes at the cleft exit but only requires that far from the cleft exit one has to achieve a uniform concentration $C_{\mathrm{i}}=C_{\mathrm{A}}=J_{\mathrm{s}} / J_{\mathrm{v}}$ since there is no region $\mathrm{B}$ in Fig. 3. In Weinbaum ${ }^{219}$ and $\mathrm{Hu}$ et al. ${ }^{78}$ a simple realistic model is developed for this mixing process in region $\mathrm{B}$. The flow at the cleft exit is treated as a line source whose velocity and concentration vary in the $y$ direction and transport equations for the radial and axial spread of the cleft exit jets are developed which satisfy appropriate matching conditions with the twodimensional solutions in the cleft.

In Fig. 5a we see the steady state pressure distribution for the case where $\mathrm{P}_{\mathrm{c}}=15 \mathrm{~cm} \mathrm{H}_{2} \mathrm{O}$ and a small negative tissue pressure of $-2 \mathrm{mmHg}$ due to tissue matrix expansion. The pressure profiles behind the GCX at $x=0$ and the cleft exit $x=0$ differ only slightly indicating that that there is hardly any water flux in the cleft except for a very low filtration since the pressure at $x=0$ is slightly higher than the cleft exit. The concentration profiles in Fig. 5b look very similar to those for $\mathrm{P}_{\mathrm{C}}=43 \mathrm{~cm} \mathrm{H}_{2} \mathrm{O}$ in Fig. $4 \mathrm{~b}$ with the important exception that the dimensionless cleft exit concentration at $x=400 \mathrm{~nm}$ is now close to 0.32 . The drop in concentration across the junction strand more distant from the junction strand breaks is again about $3 \%$. Since the experiment starts with the tissue side in pure Ringer solution it is evident that the transition to steady state, which takes several minutes, occurs through a process where initially after the rapid drop in pressure from $43 \mathrm{cmH}_{2} \mathrm{O}$ to $15 \mathrm{~cm} \mathrm{H}_{2} \mathrm{O}$ there is reabsorption which brings water into cleft where the GCX acts as a molecular sieve causing the protein concentration behind the GCX to increase until there is a near equilibrium of hydrostatic and oncotic pressures across the GCX. This is clearly seen in Fig. 5c where the concentration across the cleft is shown when this equilibrium is achieved. One notes that along the center line of the junction strand break there is a small positive gradient directed toward $x=0$. In the plasma the sum of the COP $26 \mathrm{~cm} \mathrm{H}_{2} \mathrm{O}$ and hydrostatic pressures $15 \mathrm{~cm} \mathrm{H}_{2} \mathrm{O}$ is a net $9 \mathrm{~cm} \mathrm{H}_{2} \mathrm{O}$ whereas behind the GCX at $x=0$ the average oncotic pressure is roughly $0.34 \pi_{\mathrm{p}}$ or $9.5 \mathrm{~cm} \mathrm{H}_{2} \mathrm{O}$ providing for a very small filtration through the junction strand breaks.

In summary, the steady state solutions at $P_{\mathrm{c}}=43 \mathrm{~cm} \mathrm{H}_{2} \mathrm{O}$ and $P_{\mathrm{c}}=15 \mathrm{~cm} \mathrm{H}_{2} \mathrm{O}$ in Figs. 4 and 5 show that concentration gradients behind the GCX within the cleft are very small because the diffusional resistance in the GCX overwhelms that in the cleft itself and at the low filtration pressure a steady state reabsorption cannot be achieved because the back flow from the tissue will cause the concentration behind the GCX to rise to a value that is nearly equal to that in the lumen and a very low level of positive filtration is achieved.

\section{Why Does Michel's 1-D Model Work?}

The results in Figs. 4 and 5 raise the critical question. Why does the simple one-dimensional model proposed in Michel ${ }^{133}$ provide such good agreement in the steady state data in Fig. 2 when it neither describes 
the transport in the cleft or the mixing at the cleft exit? The answer to this is that the model contains one degree of freedom, the permeability coefficient $\mathrm{P}_{\mathrm{s}}$ that appears in the Peclet number $P e=\left(J_{\mathrm{v}} / A\right)(1-\sigma) / P_{\mathrm{s}}$ in Michel's equation

$$
\frac{J_{\mathrm{v}}}{A}=L_{\mathrm{p}}\left[\Delta P-\sigma^{2} \pi_{\mathrm{p}} \frac{\left(1-e^{-P e}\right)}{\left(1-\sigma e^{-P e}\right)}\right] .
$$

By choosing the desired value of $P_{\mathrm{s}}$ or Peclet number $P e$ one can obtain an optimum fit for the steady state curve in Fig. 2. In essence this one parameter captures the combined resistance of the GCX, the cleft and the mixing region B in Fig. 3 of the two-dimensional model in Weinbaum ${ }^{219}$ and $\mathrm{Hu}$ et al. ${ }^{78}$

\section{What Happens If the Tissue is Back Loaded or There is a Vesicular Pathway?}

While Michel's transient and steady state experiments in Fig. 2 are very insightful they were performed
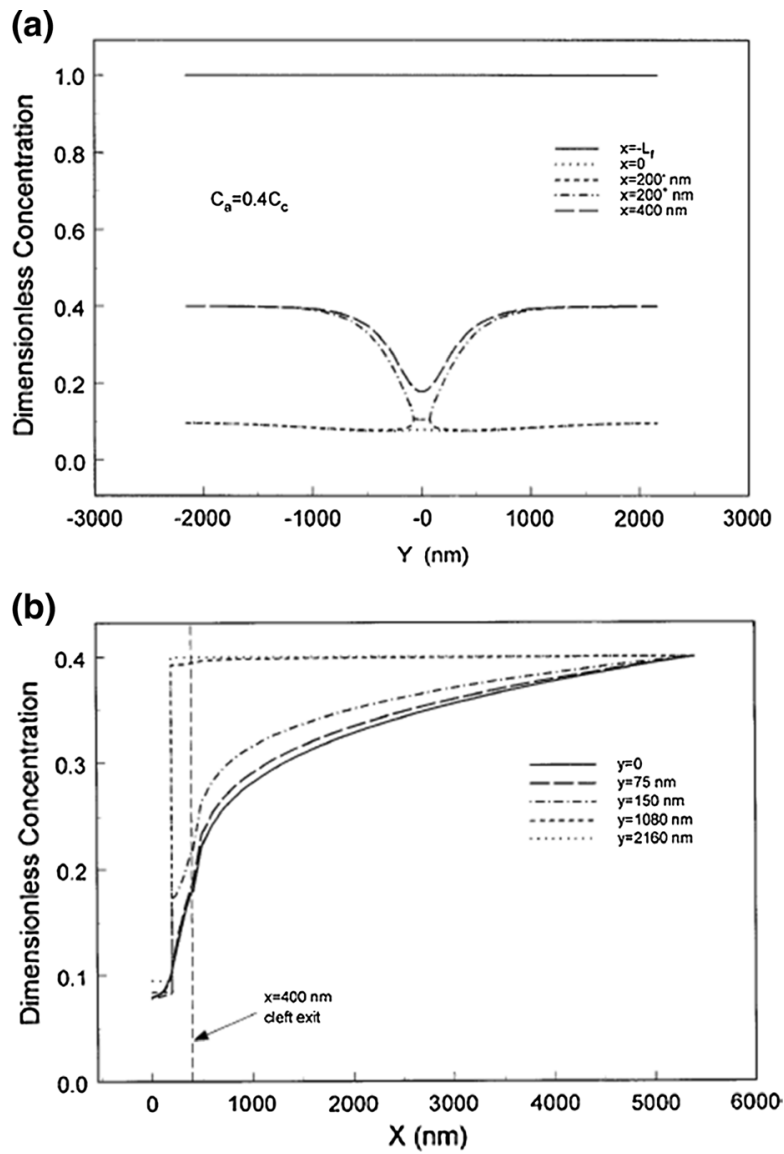

FIGURE 6. Predictions of theoretical model for $P_{\mathrm{c}}=43 \mathrm{~cm}$ $\mathrm{H}_{2} \mathrm{O}$ for concentration profiles in the cleft along $x(a)$ and along $y(b)$ when there is a parallel non-convective pathway for albumin which increases the tissue concentration at the edge of region $\mathrm{B}, C_{\mathrm{a}}$, to $0.4 C_{\mathrm{c}}$. Flow geometry is shown in Fig. 3. Plasma oncotic pressure is $26 \mathrm{~cm} \mathrm{H} \mathrm{H}_{2} \mathrm{O}$ and $\sigma=0.94$. Reprinted from Ref. 78. in only one species, frog, and in one tissue, mesentery. In marked contrast the in vivo results in Levick, ${ }^{107}$ where the classic Starling principle was being challenged, included skin, muscle and lung tissue in humans and several animal species. For many of these tissues the solute concentration in the far field in region $\mathrm{C}$ in Fig. 3 is typically $0.4 C_{\mathrm{L}}$. The solutions for the concentration profiles within the cleft for this case are shown in Fig. 6a and 6b. While the cleft and junction strand geometry is typical of frog mesenteric venules, the only available cleft geometry at the time, subsequent detailed reconstructions of rat mesenteric venules by Adamson et al. ${ }^{1}$ revealed a quite similar structure. The results in Fig. $6 \mathrm{a}$ and $6 \mathrm{~b}$ are for a high filtration state where $P_{\mathrm{L}}=43 \mathrm{~cm} \mathrm{H}_{2} \mathrm{O}$.

The results in Fig. 6a differ greatly from the concentration profiles in Fig. $4 \mathrm{~b}$ where at the same lumen pressure the concentration is nearly uniform throughout the cleft and satisfies the convective limit $(1-\sigma) C_{\mathrm{L}}$. The basic difference is that all the solute passes through the breaks in the junction strand in Fig. $4 \mathrm{~b}$ whereas in the present case there is another transendothelial pathway for large solutes which is non-convective, e.g. vesicular transport. There is a large increase in concentration across the junction strand and the concentration at the cleft exit is close to $0.4 C_{\mathrm{L}}$ except in the vicinity of the junction strand break. Here the convective flow through the break greatly impedes back diffusion from the tissue into the protected region between the GCX and the front part of the junction strand. Because of the large difference in concentration across the junction strand the concentration behind the GCX can differ greatly from that in the tissue and the classical Starling Eq. (1) no longer applies.

These results are more easily understood if one looks at the concentration profiles in Fig. $6 \mathrm{~b}$ at fixed values of $y$ from $x=0$ to the far field at $x=5 \mu \mathrm{m}$, the edge of region B where the concentration is $0.4 C_{\mathrm{L}}$. For values of $y$ removed from the junction strand break the concentration behind the GCX is uncoupled from that in the tissue and on the centerline of the break, $y=0$, where the $C$ profile is continuous, the dimensionless concentration increases from 0.08 at $x=0$ to 0.18 at the cleft exit. This same behavior will be observed if the tissue is back loaded as we discuss next.

The behavior just described was convincingly demonstrated in both frog ${ }^{77}$ and rat mesenteric venules ${ }^{1}$ where the tissue was back loaded so that the lumen and tissue concentration were both $50 \mathrm{mg} / \mathrm{mL}$. In addition, serial section microscopy was performed to determine the location and spacing of the junction strand breaks so that the $2 \mathrm{D}$ model in Weinbaum ${ }^{219}$ could be rigorously applied. In both experiments the 


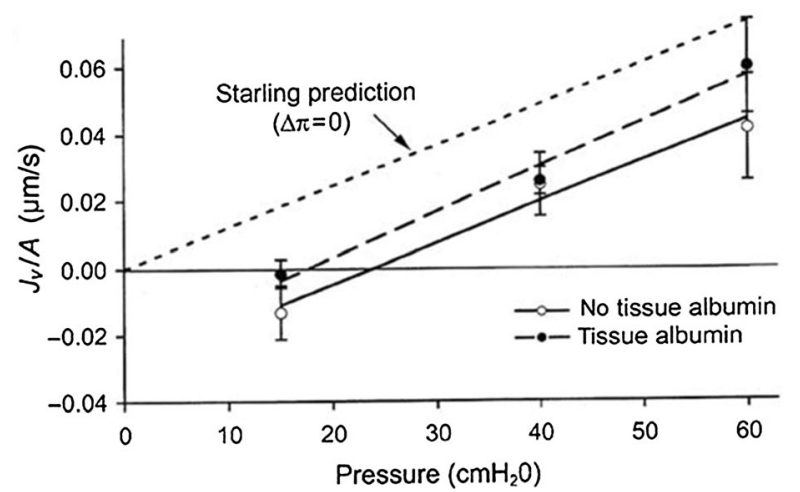

FIGURE 7. Fluid exchange in single rat mesenteric venules at controlled microvascular pressure, measured by the modified Landis red cell method (transient state). The lumen was perfused with albumin solution and the exterior was superfused with saline ('no tissue albumin', open symbols) or the same albumin solution as in the lumen ('tissue albumin', filled symbols). Short dashes show the expected increase in filtration rate for the latter according to the classic Starling principle. The much smaller observed response was as predicted by the glycocalyx-cleft model. Reprinted from Ref. 108.

lumen was perfused with albumin and the exterior of the mesentery was superfused with saline or the same albumin solution as in the lumen. The experiments for rat mesentery are shown in Fig. 7.

The short dashed curved represents the classical Starling prediction when $\pi_{\mathrm{L}}=\pi_{\mathrm{i}}$. The longer dashed curve represents the results with tissue albumin at the same concentration as the lumen. For frog mesentery ${ }^{77}$ there was very little change in the filtration rate in marked contrast to the classical Starling prediction but in good agreement with the two-dimensional Weinbaum model in $\mathrm{Hu}$ et al. $^{78}$ In the refined series of experiments with rat mesenteric venules shown in Fig. 7 (Fig. 4b in Levick and Michel ${ }^{108}$ ) the increase in $\mathbf{J}_{\mathrm{v}}$ due to raising $\pi_{\mathrm{i}}$ from zero to plasma level, was only $30 \%$ of that predicted by the classic Starling equation. Measurement of filtration across confluent cultured bovine endothelium in a Ussing chamber where the oncotic pressure was either the same or different in the luminal and abluminal reservoirs by Pang and Tarbell ${ }^{160}$ have given results very similar to those just described. At the time these tissue culture experiments were performed the role of heparinase in compromising the GCX had not been performed.

\section{Pericytes and Enclosed Microdomains}

We close this section with what is probably the most important mystery in the Michel and Phillips ${ }^{135}$ paper, the time scale over which the transition from the transient to the steady curves in Fig. 2 occurs. This transition was completed in a time that was typically less than 2 min. Using the simplified 1D model in

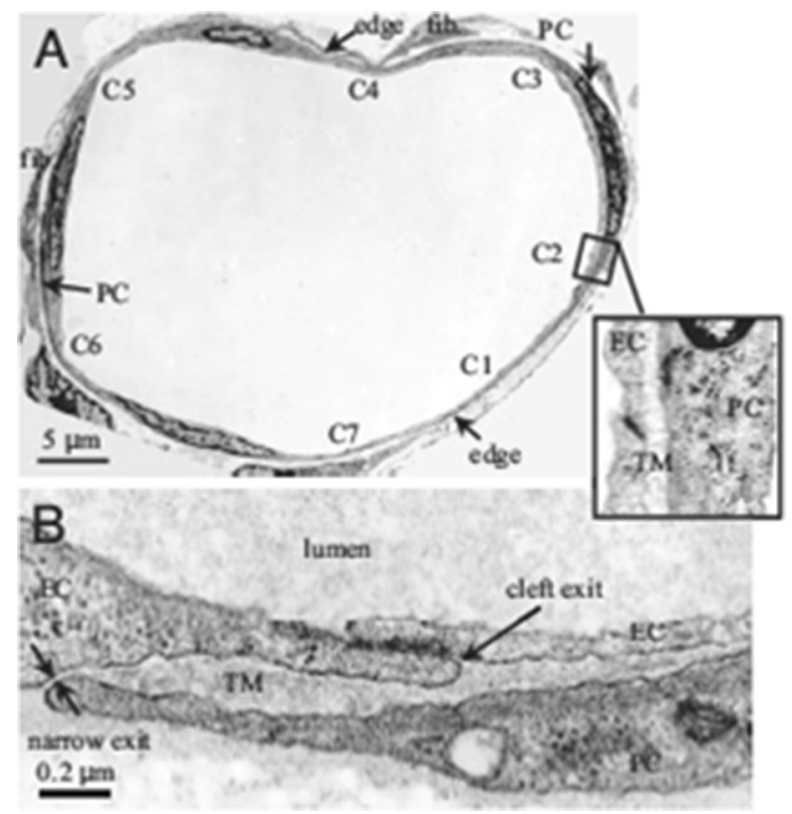

FIGURE 8. Electron microscope images of a rat venular microvessel (VM). (a) Cross-section of a VM in rat mesentery. There are seven endothelial cell (EC) clefts, C1C7, with all of the cleft exits covered by neighboring pericytes (PCs). PCs are immediately underneath ECs. There are two fibroblasts (fib.) outside PCs. The blowout shows C2 covered by a PC with a trapped microdomain (TM) of uniform thickness in between. (b) An enlarged view showing a cleft exit covered by a PC, creating a TM of roughly uniform thickness except at the narrow exit. Reprinted from Ref. 242.

Zhang et al., ${ }^{241}$ one can estimate both $J_{\mathrm{v}} / A$ and $\mathrm{J}_{\mathrm{S}}$ and calculate how long it would take the tissue concentration $C_{i}$ to change. The velocity in the transient state at the cleft exit when the pressure is dropped from 43 to $15 \mathrm{~cm} \mathrm{H}_{2} \mathrm{O}$ at the start of reabsorption is $-5.6 \mu \mathrm{m} /$ $\mathrm{s}$ and slowly rises to $0.2 \mu \mathrm{m} / \mathrm{s}$ when the new steady state of low filtrations is achieved (see Fig. 1 in Zhang et al. ${ }^{242}$ ). There is a 500 fold expansion in area from the cleft exit to the surrounding tissue so the average velocity in the tissue surrounding the cleft exit is roughly 0.002 times the cleft exit velocity or $\sim 6 \mathrm{~nm} / \mathrm{s}$. The tissue itself is filled with water that has entered during the high filtration state and its concentration is close to the convective limit which we showed in Fig. 4 is $0.07 C_{\mathrm{L}}$. The half spacing of the mesenteric venules is typically $100 \mu \mathrm{m}$ and it is this tissue volume which needs to be drained to achieve the new steady state of slow filtration. Using the above estimate of the water velocity in the tissue this would take approximately $5 \mathrm{~h}$ and not two minutes and yet the concentration in the cleft has risen from $0.07 C_{\mathrm{L}}$ to $0.32 C_{\mathrm{L}}$ at the cleft exit. A similar calculation for the solute would show that it would take 6 days for the solute flux from the cleft to raise the tissue concentration from 3 to $25.6 \mathrm{mg} / \mathrm{mL}$. This is impossible unless something fundamental has 
(a)

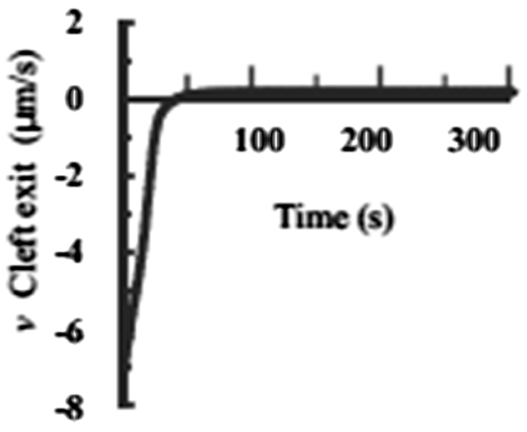

(b)

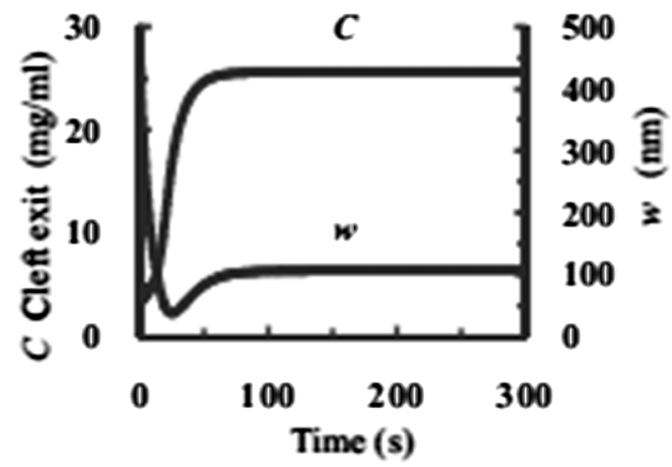

FIGURE 9. Time-dependent change in $v, C$, and $w$ at the cleft exit. Velocity $v$ (a) and concentration $C$ (b Left) and thickness $w$ of

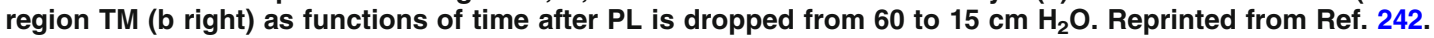

been left out of the analysis. This basic paradox was finally explained in Zhang et al. ${ }^{242}$

In the same way that there is a small protected region or enclosed microdomain between the rear of the GCX and the front of the tight junction strand, there is a similar enclosed microdomain at the cleft exits of rat mesenteric venules as shown in Fig. 8. These venules which are typically about $40 \mu \mathrm{m}$ in diameter have typically 7-8 cleft exits along a vessel cross-section. Approximately $85 \%$ of these cleft exits are covered by pericytes (PC) which cover much of the surface of these venules. As shown in the enlarged image in Fig. $8 \mathrm{~b}$ there is an enclosed trapped microdomain (TM) formed by the pericytes surrounding the cleft exits whose thickness can vary between 100 and $500 \mathrm{~nm}$ depending on the state of hydration. At the edge of the TM there is an attachment region or adherens junction between the EC membrane and the edge of the PCs. The TM region is very similar in structure to the region between the EC layer in aortic endothelium and the internal elastic lamina which also has been shown to vary in thickness by about fivefold depending on the pressure in the lumen and state of hydration. ${ }^{80,81}$

Zhang et al. ${ }^{242}$ have developed an elastohydrodynamic model for the TM region observed in Fig. 8. The key results in this model are shown in Fig. 9a and 9b. One observes in Fig. 9a how the fluid drains from the TM in the case when $P_{\mathrm{L}}$ has been quickly dropped from $60 \mathrm{cmH}_{2} \mathrm{O}$ to $15 \mathrm{cmH}_{2} \mathrm{O}$ and a new steady state allowed to develop. One notes for this case that the cleft exit velocity increases from $-7.4 \mu \mathrm{m} / \mathrm{s}$ reabsorption to $0.2 \mu \mathrm{m} / \mathrm{s}$, a slow rate of steady filtration. In Fig. 9b we see how the concentration in the TM increases from $3 \mathrm{mg} / \mathrm{mL}$ to $25.6 \mathrm{mg} / \mathrm{mL}$ as the fluid drains out of the TM and the change in TM thickness W from $500 \mathrm{~nm}$ to roughly $100 \mathrm{~nm}$ due to this fluid reabsorption. The key observation is that the time scale of this transition process is typically one minute, very similar to the classic paper of Michel and Phillips, ${ }^{135}$ and not hours. The experiments in Michel's paper were conducted in frog mesenteric venules whereas the model results in Fig. 9 were based on the rat mesenteric venules shown in Fig. 8.

The model just described applies to tissues where a large fraction of the EC clefts are covered by pericytes and filtration occurs primarily through the clefts. However, the model can also be applied to skeletal muscle if there are small regions where water can accumulate. Clinically it has been observed that reabsorption can continue in human skeletal muscle for 20 30 min after a sudden drop in $P_{\mathrm{L}}$ as would occur after hemorrhage. ${ }^{107}$ Typical reabsorption when $P_{\mathrm{L}}$ was reduced $5 \mathrm{mmHg}$ was $15-30 \mathrm{~mL}$ per $\mathrm{kg}$ of tissue in $30 \mathrm{~min}$. Thus, reabsorption of between 0.3 and $0.6 \mathrm{~L}$ is expected from $20 \mathrm{~kg}$ of human muscle in $20-30 \mathrm{~min}$. This reabsorption would produce a $15-20 \%$ increase in interstitial concentration. This is enough to slow but not stop further reabsorption and is shorter than values of an hour or more that would be required to produce changes in the total ISF volume.

In summary, we have tried to highlight in this section the fundamental differences between the one-dimensional model in Michel ${ }^{133}$ and the two-dimensional model in Weinbaum ${ }^{219}$ and $\mathrm{Hu}$ et al. ${ }^{78}$ where the detailed effect of the cleft and its junction strand can be examined as well as the mixing process at the cleft exit. We have also tried to explain why Michel's one-dimensional model works so well in fitting the steady state results in Michel and Phillips ${ }^{135}$ in Fig. 2. The mixing process at the cleft exit is of particular significance when the tissue concentration is raised by a nonconvective parallel pathway. We also explored the role that pericytes at the cleft exit play in determining the time scale of the transition from transient to steady state behavior, as might occur in muscle tissue when 
there is a sudden drop in capillary pressure due to hemorrhage, if there are local regions of trapped water in the vicinity of the microvessels.

This opening section of Part A, on the revised Starling Principle, has begun to reveal how vascular physiology is influenced by the GCX. This theme continues in the next section on Mechanotransduction with its emphasis on the GCX as a mechanosensor for shear-induced nitric oxide production and substrate stiffness-induced endothelial dysfunction associated with loss of GCX. The final section of Part A describes new insights into GCX structure obtained through high resolution microscopy that will contribute to future advances in cardiovascular physiology.

\section{Mechanotransduction}

An important physiological function of the GCX is to serve as a mechanosensor and transducer. $^{61,69,200-202,220}$ It is well known that a dysfunctional endothelium is an early manifestation of atherosclerosis $^{234}$ and that in disease prone locations such as branches and curvatures, the flow departs from pulsatile, unidirectional shear stress to create flow separation zones that include low wall shear stress, flow reversal, oscillatory and multi-directional shear stress, and sometimes turbulence (chaotic flow). ${ }^{39,41,247}$ In contrast, in adjacent, non-diseased regions, shear stress is higher and flow is unidirectional with minimal reversal. The GCX plays an important role in EC mechanotransduction of shear stress that mediates localization of atherosclerosis. Weinbaum et al. ${ }^{221}$ pointed out that the existence of the GCX could reduce the fluid shear stress on the vascular EC surface to a negligible level, while Secomb et al. ${ }^{185}$ described the transfer of fluid mechanical shear stress at the interface between the fluid and the outer regions of the GCX, to solid mechanical stress within the matrix that is transmitted to the cell body. Thi et al. ${ }^{205}$ further showed that the GCX is required for the EC cytoskeleton to respond to shear stress.

It is well established that after $24 \mathrm{~h}$ of laminar flow, ECs align in the direction of flow and their proliferation is suppressed. Yao et al. ${ }^{229}$ removed HS from EC in vitro using the specific enzyme heparinase III and observed that EC no longer aligned under flow after $24 \mathrm{~h}$ and they proliferated as if there were no flow present. Subsequently, Baeyens et al. ${ }^{7}$ reported that EC in the thoracic aortas of syndecan 4 (SDC4) null mice were poorly aligned in the direction of the flow. They found that depletion of SDC4 in human umbilical vein ECs (HUVECs) using shRNA also inhibited flow-induced alignment in vitro, which was rescued by re-expression. In hypercholesterolemic mice, they observed that deletion of SDC4 drastically increased atherosclerotic plaque burden with the appearance of plaque in normally resistant locations.

\section{Shear-Induced Nitric Oxide Production}

Effects of Enzyme Degradation of GCX on NO Production

Florian et $a l .{ }^{60}$ showed that enzymatic removal of HS from the surface of cultured EC in vitro with heparinase III completely blocked shear-induced NO production in both steady flow $\left(15 \mathrm{dyn} / \mathrm{cm}^{2}\right)$ and reversing oscillatory flow $\left(10 \pm 15 \mathrm{dyn} / \mathrm{cm}^{2}\right)$ out to $3 \mathrm{~h}$ exposure. These experiments included both the early phase (seconds to minutes after a step increase in shear) that is calcium and G-protein dependent as well as the later phase (minutes to hours) that is independent of calcium and G-protein. ${ }^{98}$ Yen et al. ${ }^{231}$ later demonstrated in vivo that degradation of HS in the GCX of rat mesenteric microvessels greatly inhibited $\mathrm{NO}$ production in response to an increase in flow. Pahakis et al. ${ }^{159}$ showed that shear-induced NO production was blocked by enzymes removing HS and HA, but not CS and that these treatments had no effect on the shear-induced production of the important vasodilator and antiplatelet agent, prostacyclin $\left(\mathrm{PGI}_{2}\right)$.

In related work, Dull et al. ${ }^{48}$ examined the transmural pressure vs. hydraulic conductivity $\left(L_{\mathrm{p}}\right)$ relationship in lung microvascular ECs and demonstrated that HS participates in pressure-sensitive mechanotransduction that results in EC transport barrier dysfunction. Note that increased transmural pressure drives increased flow through intercellular junctions and over the nearby EC surfaces. Pretreatment of lung capillary EC with heparinase III completely abolished the pressure-induced increase in $L_{\mathrm{p}}$ and reduced intracellular NO detected by a fluorescent dye, suggesting that cell-surface HS directly participates in mechanotransduction resulting in NO production and increased $L_{\mathrm{p}}$. In a related in vivo study Dull et al. ${ }^{47}$ examined the roles of HS and NO in pressure induced alterations of the capillary filtration coefficient, $\mathrm{K}(\mathrm{fc})$, and found that heparinase III attenuated the effects of increased capillary pressure on $\mathrm{K}(\mathrm{fc})$.

\section{GCX Core Proteins Involved in NO Production}

Because the proteoglycan core protein glypican-1 (GPC1) only binds HS and not CS and the dominant apical syndecan (syndecan-1; SDC1) binds both HS and CS, it was hypothesized that GPC1 is the core protein that transmits the fluid shear force sensed by the HS to the cell surface where it is ultimately transduced via the phosphorylation of endothelial nitric oxide synthase (eNOS) into NO. This hypothesis was tested by Ebong et al. ${ }^{50}$ who showed that knockdown 
of GPC1 using shRNA completely blocked shear-induced phosphorylation of eNOS, whereas knockdown of SDC1 had no effect. Because knockdown of genes by shRNA can have off-target effects, the hypothesis was further tested using atomic force microscopy (AFM) with cantilevers coated with specific antibodies to HS, GPC1, and SDC1. ${ }^{12}$ Force applied to HS or GPC1 resulted in NO production, whereas force applied to SDC1 did not. These experiments were carried out at 10 and $30 \mathrm{~min}$ and did not specifically interrogate the early G-protein-dependent phase of activation. Zeng and $\mathrm{Liu}^{237}$ found that shear stress has a dual role in eNOS activation: $4 \mathrm{dyn} / \mathrm{cm}^{2}$ shear stress inhibited the activation of eNOS, and $15 \mathrm{dyn} / \mathrm{cm}^{2}$ shear stress induced it. Removal of GPC1 by phosphatidylinositol phospholipase C (PI-PLC) significantly suppressed the shear stress-induced eNOS activity at $15 \mathrm{dyn} / \mathrm{cm}^{2}$ shear and further reduced the 4 dyn $/ \mathrm{cm}^{2}$-inhibited eNOS activity. ${ }^{237}$

\section{Glypican-1 and PECAM-1 Interact in NO Production}

Recent work has shown that ECs transfected with platelet endothelial cell adhesion molecule-1 (PECAM1) siRNA lose their inherent sensitivity to flow in the production of NO. ${ }^{59,226}$ In both bovine aortic ECs (BAECs) and HUVECs, the absence of PECAM-1 protein decreased the phosphorylation of eNOS at ser1177 and production of NO after $30 \mathrm{~min}$ of flow. ${ }^{59,226} \mathrm{Xu}$ et al. ${ }^{226}$ found that flow-mediated eNOS phosphorylation in vivo induced by voluntary wheel running was reduced in PECAM-1 knockout mice. These findings implicate PECAM-1 as an important regulator of shear-induced NO release in ECs, although they do not clarify whether PECAM-1 directly senses flow to initiate these processes or responds to an upstream sensor of fluid shear stress.

In many cases, PECAM-1 works in conjunction with other mechanisms to sense changes in extracellular fluid flow. ${ }^{43,125,208} \mathrm{HS}$ and GPC1 cluster via mobile lipid rafts at cell-cell junctions, where the majority of PECAM-1 is located, after $30 \mathrm{~min}$ of fluid shear stress application. ${ }^{239}$ A HS-to-PECAM-1 binding complex has been shown to exist ${ }^{29,33}$ and participate in early phase endothelial mechanotransduction. ${ }^{43}$ Human coronary artery ECs (HCAECs) treated with heparinase III or reconstituted with PECAM-1 mutants lacking HS binding domains had inhibited (early phase) shear-induced signaling, specifically Akt phosphorylation. ${ }^{43}$

In addition to forming binding complexes with its extracellular Ig-like domains, PECAM-1 can also participate in signaling cascades by activation of its intracellular tail. PECAM-1 has two highly conserved tyrosines, 686 and 663, that become phosphorylated in response to mechanical force, including physiologic levels of fluid shear stress in ECs. ${ }^{125,157}$ PECAM-1 phosphorylation is mediated by Src family kinases, specifically c-Src. ${ }^{59,125,157}$ PECAM-1 activation is also regulated by recruitment of the protein tyrosine phosphatase SHP-2, which dephosphorylates PECAM-1, allowing cyclic signaling waves to stem from continuous fluid shear stress application. ${ }^{79,125}$ Importantly, blocking PECAM-1 phosphorylation using the Src kinase inhibitor PP1 completely blocks downstream shear-induced eNOS phosphorylation, ${ }^{59}$ clarifying that PECAM-1 activation is a critical step in shear-induced NO production via the phosphorylation of eNOS.

Recently, Zeng et al. ${ }^{240}$ described the relationship between GPC1 and PECAM-1 in shear-induced production of NO. Using AFM methods on HUVECs and rat fat pad ECs (RFPECs) in vitro for $30 \mathrm{~min}$, they showed that force applied directly to GPC1 induces NO production while force applied directly to PECAM-1 does not. When PECAM-1 was knocked down by siRNA, force applied directly to GPC1 did not induce NO production. Furthermore, using GPC1 knockout mice, Bartosch (unpublished data) demonstrated lower NO production than the wild type mice under chronic flow conditions with no differences in PECAM-1 expression. Under acutely increased flow stimulated by administration of dobutamine that elevates heart rate, there was an upregulation of $\mathrm{NO}$ production in the wild type mice after $30 \mathrm{~min}$, but no increase in the knockout mice. These results demonstrate that GPC1 is the upstream shear sensor and PECAM-1 is the downstream effector.

But how does GPC1 communicate with PECAM-1? In Zeng et al. ${ }^{240}$ it was shown that blocking interactions between the HS GAG on GPC1 and the extracellular domain of PECAM-1 had no effect on signaling, while enzymatic removal of HS from GPC1 blocked phosphorylation of the intracellular domain of PECAM-1 and downstream phosphorylation of eNOS, the precursor to NO formation. The overall mechanism suggested by these studies is summarized in Fig. 10.

\section{Shear-Induced $\mathrm{PGI}_{2}$ is Mediated by PECAM-1}

To follow-up on the observation that GAG removal by enzymes did not block shear induced $\mathrm{PGI}_{2}$ production, ${ }^{159}$ Russell-Puleri et al. ${ }^{176}$ investigated primary cilia, SDC4, and PECAM-1, as potential mechanosensors for $\mathrm{PGI}_{2}$ production. Primary cilia are localized to the apical surface of EC and SDC4 to the basal surface. In confluent ECs PECAM-1 localizes at intercellular junctions where it regulates homophilic binding between cells. Separate knockdown of the 


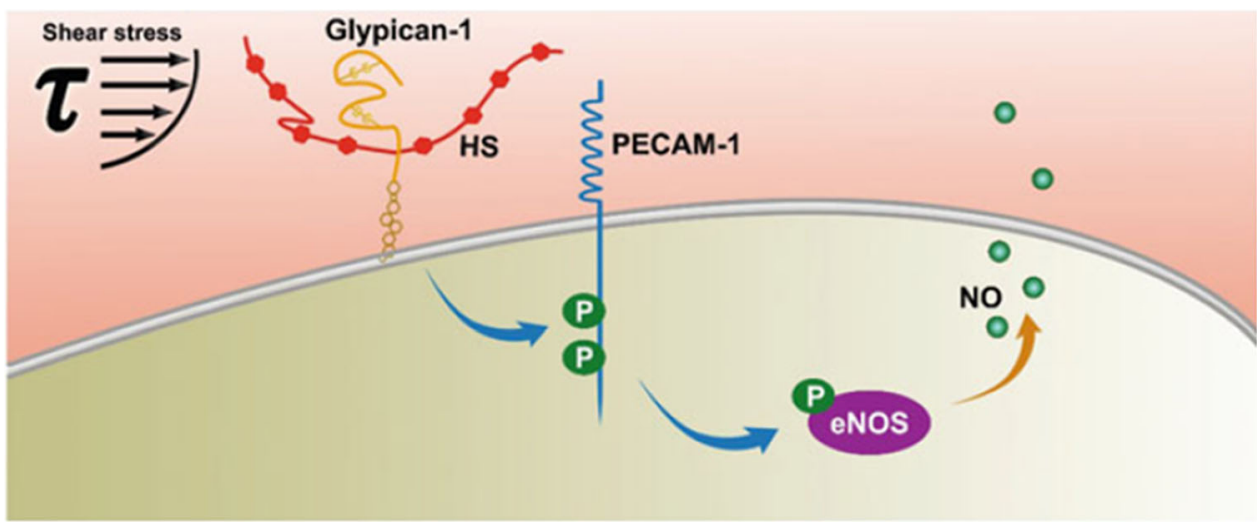

FIGURE 10. Shear-Induced Nitric Oxide Production via Heparan Sulfate and Glypican-1 Mechanotransduction and PECAM-1 Phosphorylation leading to eNOS activation and NO synthesis. Fluid shear stress is initially sensed by heparan sulfate proteoglycan glypican-1. That signal is transduced to the intracellular tail of PECAM-1 protein. PECAM-1 becomes activated by tyrosine phosphorylation. PECAM-1 activation triggers phosphorylation of eNOS and NO production increases. Reprinted from Ref. 240.

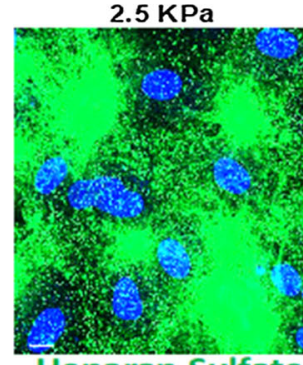

Heparan Sulfate

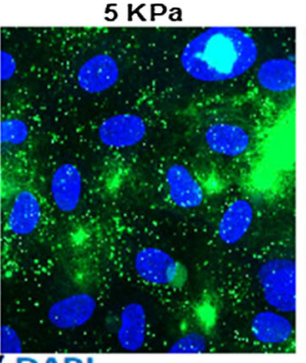

DAPI
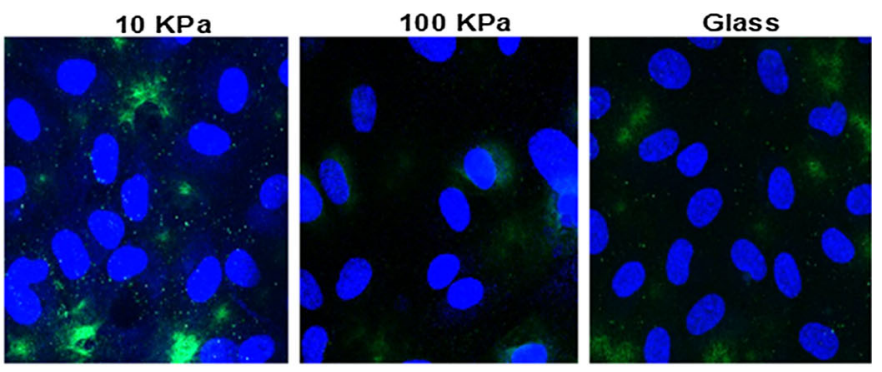

FIGURE 11. Substrate stiffness inhibits the glycocalyx GAG heparan sulfate. HUVECs were cultured on polyacrylamide gels of stiffness ranging from $2.5 \mathrm{kPa}$ (soft gel) through $10 \mathrm{kPa}$ (stiff gel) up to $100 \mathrm{kPa}$. Heparan Sulfate (green) expression was assessed by immunostaining. Cell nuclei were identified using DAPI. Reprinted from Ref. 120.

three putative mechanosensors in cultured cells revealed that only PECAM-1 deletion blocked shearinduced $\mathrm{PGI}_{2}$ production and upregulation of the enzyme cyclooxygenase 2 (COX-2) by a mechanism involving inside-out activation $\alpha_{5} \beta_{1}$ integrin. The role of PECAM-1 in shear-induced $\mathrm{PGI}_{2}$ was further confirmed in a PECAM-1 knockout mouse model. ${ }^{176}$ Conway et al..$^{32}$ using a fluorescence resonance energy transfer (FRET) tension sensor, showed that shear stress increased tension across PECAM-1 at intercellular junctions, suggesting a mechanism by which shear stress activates PECAM-1.

\section{Substrate Stiffness Suppresses the Glycocalyx}

With increased age, or as a result of hypertension, arteries lose elasticity and thicken, giving rise to a stiffened arterial wall and substrate for ECs. ${ }^{140,217}$ Arterial stiffness is a key underlying risk factor for, and is a hallmark of, age-related cardiovascular diseases such as atherosclerosis and stroke. ${ }^{141}$ EC dysfunction, characterized by increased proliferation, permeability, and inflammation is at the heart of the mechanisms driving these age-mediated vascular diseases. Arterial stiffness is a key trigger for EC dysfunction to occur. ${ }^{49,124}$

In a recent study, Mahmoud et al. ${ }^{120}$ showed that EC grown on polyacrylamide (PA) gels of substrate stiffness $10 \mathrm{kPa}$ (mimicking the subendothelial stiffness of aged, unhealthy arteries) displayed a significant inhibition of GCX expression compared to cells cultured on softer PA gels $(2.5 \mathrm{kPa}$, mimicking the subendothelial stiffness of young, healthy arteries). The effect of stiffness on HS immunostaining is displayed in Fig. 11. Gene and protein screens revealed that a GCX core protein, GPC1, was inhibited in cells cultured on stiff gels.

Cells grown on $10 \mathrm{kPa}$ gels had enhanced EC dysfunction as determined by enhanced cell inflammation (enhanced inflammatory gene expression, monocyte adhesion and inhibited eNOS phosphorylation), proliferation and endothelial mesenchymal transition (EndMT). Removal of GPC1 using gene-specific silencing with siRNA or gene overexpression using a plasmid revealed that GPC1 is required to protect against stiffness-mediated EC dysfunction. Consistent 
with this, using a model of age-mediated stiffness, older mice exhibited a reduced expression of GPC1 and enhanced EC dysfunction compared to young mice. EC dysfunction was exacerbated in young, but not aged, GPC1 knockout mice.

\section{Structural Mechanism-Ultrastructure and Organization of Endothelial GCX}

In addition to the above summarized molecular mechanisms by which GCX plays its role as a mechano-sensor and transducer, an organized structure of endothelial GCX at the molecular level is necessary. Due to the limitation of optical and electron microscopy, the ultra-structure of endothelial GCX has not been revealed until the recent development of super resolution stochastic optical reconstruction microscopy, STORM. ${ }^{14,225}$ Fan et al. ${ }^{57}$ used two photoswitchable dyes, ATTO488 and AF647, to label HS and HA of GCX on bEnd3 (mouse brain microvas- cular EC) monolayers, and revealed their organization and ultra-structure by N-STORM (Fig. 12). Figure 12 demonstrates that HA is a long molecule weaving into a network which covers the endothelial luminal surface. In contrast, HS is a shorter molecule, perpendicular to the cell surface. HA and HS are partially overlapped with each other at the endothelial luminal surface. These findings suggest that HS plays a major role in mechanosensing and HA plays a major role in the molecular sieve.

\section{PART B: THE ROLE OF THE GLYCOCALYX IN VASCULAR RELATED DISEASES}

\section{Infectious Diseases}

Since the GCX is at the interface of the circulating blood and vessel wall, in addition to experiencing the forces generated by the blood flow, it is constantly

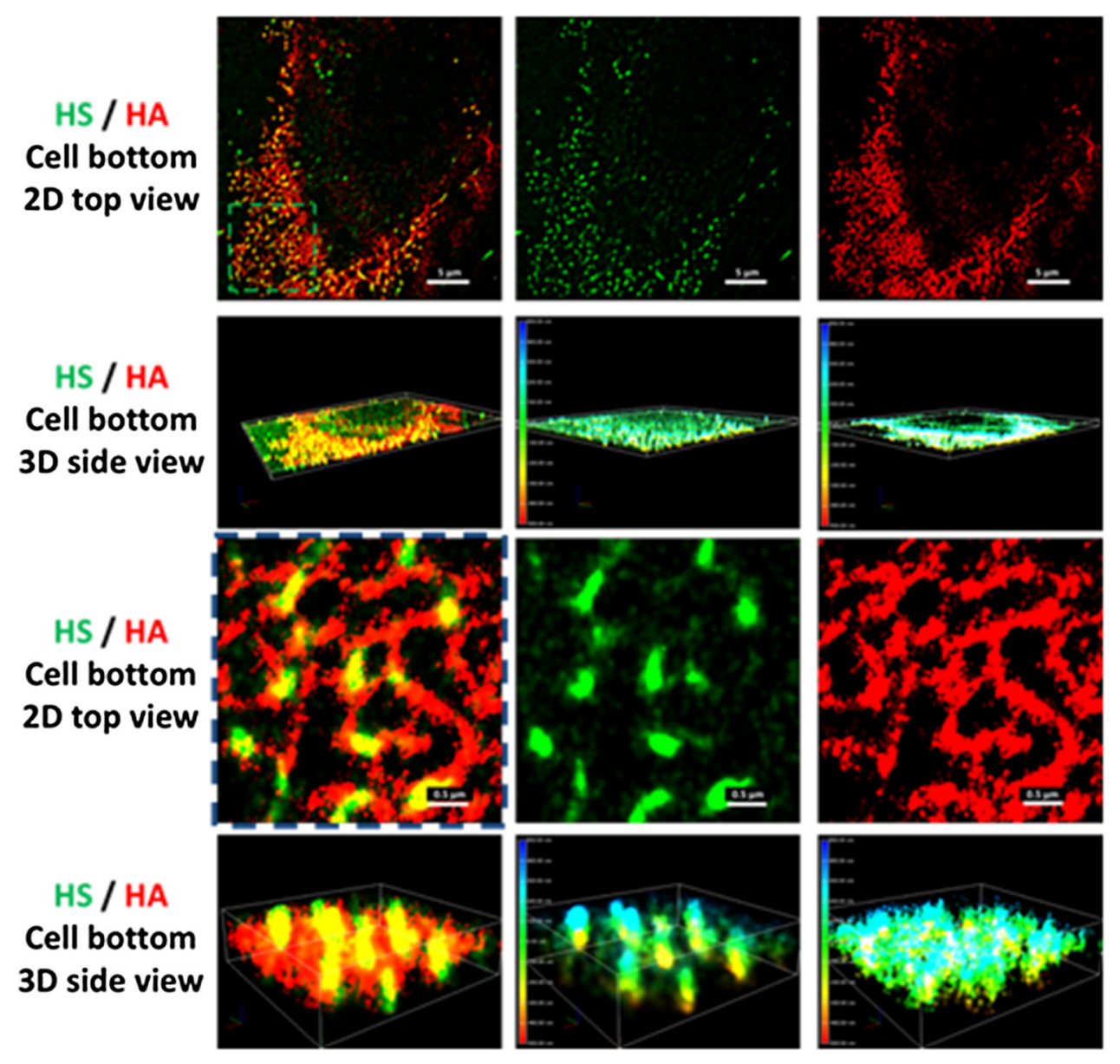

FIGURE 12. Illustrations of STORM images for HS and HA from the cell luminal surface. In the illustrations, the second column is the HS image (green), the third column is the HA image (red) and the first column is the overlay of the second and the third columns. The first row is the 2D top view and the second row is the 3D view of the field of $\sim 34 \mu \mathrm{m} \times 34 \mu \mathrm{m}$. The third and fourth rows are the enlarged 2D and 3D views from the region with the green dashed line in the first row. The color bar at the left of the 3D view for HS (second column) and HA (third column) represents the depth (length) scale. Reprinted from Ref. 57. 
affected by the blood-borne substances including toxins from infectious diseases.

\section{Sepsis}

Sepsis is a serious medical condition. It is caused by an overwhelming immune response to infection. The body releases immune chemicals (cytokines) into the blood to combat the infection. The cytokines trigger widespread inflammation, which leads to blood clots and leaky blood vessels. As a result, blood flow is impaired, and that deprives organs of nutrients and oxygen and leads to organ damage. In severe cases, one or more organs fail. In the worst cases, blood pressure drops, the heart weakens, and the patient spirals toward septic shock. Once this happens, multiple organs, lungs, kidneys, brain, may quickly fail, and the patient can die. Sepsis is a major challenge in hospitals, where it is one of the leading causes of death. It afflicts > 750,000 individuals annually in the United States alone and has mortality rates of $40-60 \% .{ }^{63}$

Sepsis occurs unpredictably and can progress rapidly. Many types of microbes can cause sepsis, including bacteria, fungi, and viruses. However, bacteria are the most common cause. One of the key molecular causes of Gram-negative bacteria induced septicemia is endotoxin that consists of lipopolysaccharides (LPSs) bound with high affinity to LPSbinding glycoprotein. Complex LPS-binding glycoprotein is recognized by cognate receptor Toll-like receptor 4 (TLR4) and co-receptor CD14 on monocytes/macrophages and ECs to induce the secretion of proinflammatory cytokines. ${ }^{3}$ Considering the systemic nature of septicemia, vascular endothelium represents the first line of exposure to bacterial endotoxins. The role of microcirculation and microvascular endothelium in the pathogenesis of septic multiorgan failure has been known for a long time, ${ }^{3}$ but recent studies have identified endothelial GCX in manifestations of endothelial dysfunction in sepsis. ${ }^{182,194,248}$

Among the earliest responses of activated ECs to endotoxin are exocytosis of Weibel-Palade bodies (WPBs) and secretory lysosomes. WPBs are rodshaped members of lysosome-related organelles ( $0.2 \mu \mathrm{m}$ by 2 to $3 \mu \mathrm{m})$ characteristic to ECs and containing an array of proteins, peptides and cytokines, which can be released quickly on urgent demand. The endothelial lysosomes contain acid/secretory sphingomyelinase, glycohydrolases, cathepsins, fucosidase, phosphatases, HS sulfatase, among others. Some of these enzymes can degrade the endothelial GCX. Upon application of LPS for minutes to simulate the onset of sepsis, confocal and STORM microscopy confirmed the patch loss of GCX simultaneously with the exocytosis of lysosome-related organelles and WPBs in cultured ECs and mouse aorta ${ }^{248}$ (Fig. 13). During sepsis, the GCX becomes thinner, inducing tissue edema. ${ }^{25,209}$ In mice, intravenous LPS injection results in elevated levels of tumor necrosis factor (TNF)- $\alpha$ and IL- $6{ }^{58}$ Elevated levels of TNF- $\alpha$ contribute to GCX damage via increased matrix metalloproteinase (MMP) activity and syndecan loss. ${ }^{173}$ In human, low-dose endotoxin resulted in a significant reduction in the depth of the sublingual vessel GCX and a concurrent elevation in plasma HA, suggesting GCX shedding. ${ }^{151}$ Circulating levels of HA and HS have also been increased fourfold in patients with sepsis, being higher in those who did not survive 90 days. ${ }^{149}$ Therefore, multiple circulating biomarkers of GCX shedding have
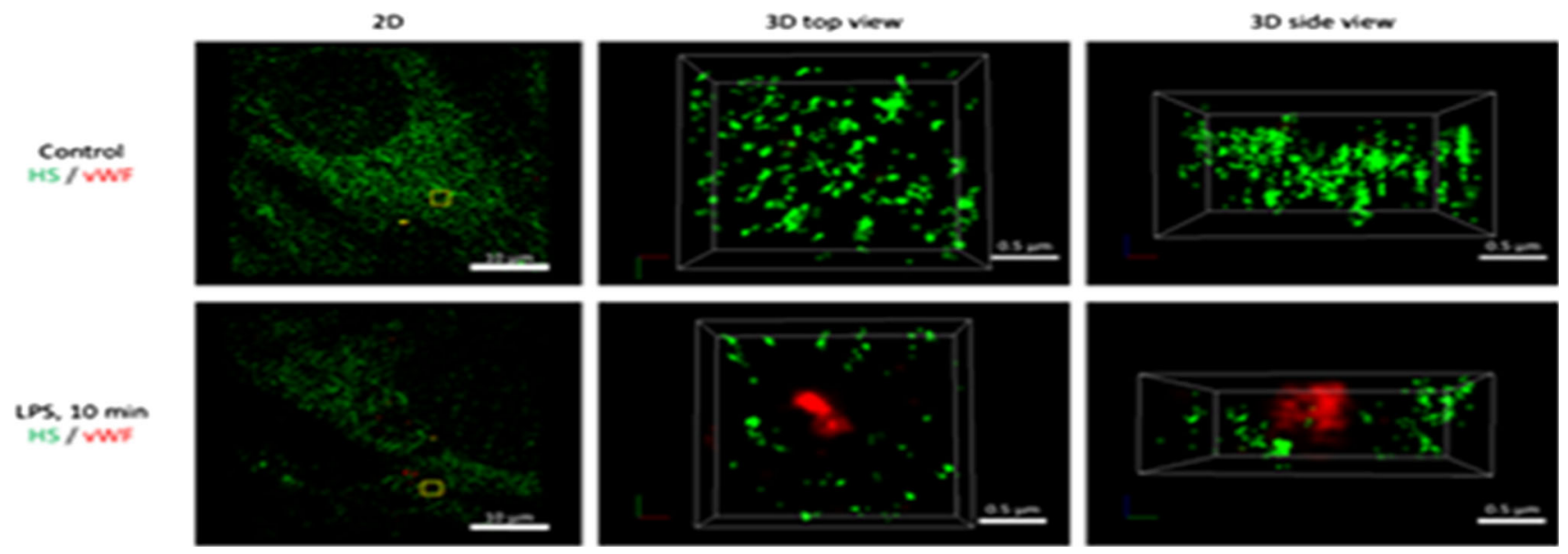

FIGURE 13. Representative STORM-acquired images of anti-HS labeled EG and vWF-labeled WPBs distribution in control and $10 \mathrm{~min}$ post-LPS. The left panel shows 2D images of a region of $\sim 40 \mu \mathrm{m} \times 40 \mu \mathrm{m}$ at the apical surface of cultured mouse brain microvascular endothelial cell (EC). An enlarged top view of a yellow box $(\sim 2 \mu \mathrm{m} \times 2 \mu \mathrm{m})$ in the left panel is shown in the middle panel and its side view in the right panel. The EC surface is at the bottom of the side view. Upper panels are for the control and bottom ones for after 10 min LPS treatment. Reprinted from Ref. 248. 
been studied in humans as markers of sepsis, including SDC1, HS and HA. ${ }^{15,36,118,227}$

Sepsis, in general, can be characterized as a catabolic state of degradation of proteins, lipids and carbohydrates. $^{215}$ Recent advanced metabolic gene expression analysis of LPS-induced endothelial dysfunction found an increase in glycan production to accompany GCX loss in ECs and an increase in endothelial permeability. ${ }^{129}$ Findings in patients with sepsis showed that upregulation of glycan synthesis was among the worst-affected areas of metabolism, being most severe in non-survivors. These observations are consistent with a view of compensatory induction of synthesis of structural components of GCX, upon application of GCX-degrading stressors such as LPS, suggesting a tight feedback control mechanism coupling its synthesis and degradation. That the degradation of GCX in sepsis prevails over increased synthesis of glycans, major components of GCX, suggests an enhanced enzymatic degradation, which is carried out by sheddases, including a disintegrin and metalloproteinases (ADAMs), MMPs, heparanases and hyaluronidases. ${ }^{63}$ While ADAM 10 is expressed constitutively, ADAM 17 is inducible, as it is converted to an active form upon cell stimulations. ${ }^{137} \mathrm{In}$ addition to turning TNF- $\alpha$ into a soluble form, ADAM 17 cleaves SDC4 while ADAM 10 sheds SDC1 and ADAM15 cleaves CD44. ${ }^{227}$ MMPs induced in sepsis activate TNF- $\alpha$, interfere in CD44-HA binding and shed HS, CS, and the SDC1 ectodomain. ${ }^{244}$

The wall of the cerebral microvessel is named the blood-brain barrier (BBB) which is formed by ECs, pericytes, basement membrane and astrocyte foot processes. The GCX on ECs is the most luminal layer of the BBB. During sepsis, patients develop cognitive impairment. Although the pathogenesis of this condition is complicated, current studies suggest a role for cytokines (IL-1, IL-6 and TNF- $\alpha$ ) penetrating the BBB and activating astrocytes. ${ }^{165}$ Studies also suggest that during sepsis HS fragments penetrate the BBB in the hippocampus to inhibit long-term potentiation, the process responsible for memory formation. ${ }^{76}$

Increased pulmonary vascular permeability occurs early in sepsis ${ }^{128}$ and may be due to GCX damage. Although a study using bovine lung microvascular ECs in vitro suggested that $\mathrm{HS}$ of GCX contributed to the barrier function, a study using ex vivo rat lungs did not. ${ }^{47,48}$ One explanation for this discrepancy is that the enzyme degrading HS completely compromised the barrier function of GCX for the in vitro lung microvascular ECs formed monolayer, but only partially compromised that of GCX in the ex vivo rat lung microvessels. In addition to HS, there is significant HA in the GCX of microvessels. As predicated by a transport model for the paracellular pathway (inter- endothelial cleft) of the microvessel wall, ${ }^{21}$ as long as there is $50 \%$ GCX left that covers the inter-endothelial cleft, the hydraulic conductivity and permeability to a large sized molecule would not have a significant increase. For a rat mesenteric microvessel, the inter-endothelial cleft occupies about $0.2 \%$ of a vessel lumen area. ${ }^{1}$ Even though $99.8 \%$ GCX is degraded from the vessel lumen, as long as $0.2 \%$ GCX still covers the inter-endothelial cleft, no significant increase in the vascular permeability would be observed. This explanation also applies to the recent finding by Guerci et al. ${ }^{64}$ that GCX degradation is independent of vascular barrier permeability increases in nontraumatic hemorrhagic shock in rats.

However, if that $0.2 \%$ GCX covering the inter-endothelial cleft is damaged, vascular permeability will be enhanced. If other structural components, such as tight and adherens junctions inside the inter-endothelial cleft and the width of the cleft are disrupted, large increases in the vascular permeability will occur. For example, a study by Schmidt et al. ${ }^{182}$ shows that immune cell extravasation and inflammation during sepsis can alter the pulmonary GCX, resulting in pulmonary edema.

Using in vivo multiphoton microscopy in mice, Butler et $a l .{ }^{20}$ observed a continuous GCX (identified by fluorescently labeled wheat germ agglutinin bound to sialic acid) at glomerular capillaries that contributes to the restriction of macromolecules and water passage from the glomerulus into the Bowman space. In a rat model, sepsis at its initial phase alters glomerular GCX and induces albuminuria (albumin leakage into urine). ${ }^{2}$ Taking these together, sepsis first destroys the frontline of the vascular barrier, the GCX, eventually resulting in multiorgan failure including brain, lungs and kidneys, to name a few.

\section{Dengue Virus}

Dengue is the most prevalent arboviral disease in humans and a major public health problem worldwide. Systemic plasma leakage, leading to hypovolemic shock and potentially fatal complications, is a critical determinant of dengue severity. A novel pathogenic effect of the secreted dengue virus (DENV) nonstructural protein 1 (NS1) is triggering hyper-permeability of human ECs in vitro and systemic vascular leakage in vivo. It has been demonstrated that DENV NS1 disrupts the GCX on human pulmonary microvascular ECs, inducing degradation of sialic acid and shedding of HS proteoglycans by a mechanism involving NS1-induced expression of sialidases and heparanase. ${ }^{168}$ The data suggest an important role for GCX shedding in Dengue disease. 
In a related study of 103 Dengue-infected patients, elevated levels of SDC1 and CS were strongly associated with plasma leakage, while elevated levels of SDC1 and claudin-5 were strongly associated with severe plasma leakage. ${ }^{196}$

\section{Flaviviruses}

Flaviviruses cause systemic or neurotropic-encephalitic pathology in humans. The flavivirus nonstructural protein 1 (NS1) is a secreted glycoprotein involved in viral replication, immune evasion, and vascular leakage during Dengue virus infection. In an extension of their dengue virus studies, Puerta-Guardo et al. ${ }^{167}$ investigated the contribution of secreted NS1 from related flaviviruses and demonstrated that NS1 from Zika, West Nile, Japanese encephalitis, and yellow fever viruses selectively binds to and alters permeability of human ECs from lung, dermis, umbilical vein, brain, and liver in vitro and causes tissue specific vascular leakage in mice, reflecting the pathophysiology of each flavivirus. Mechanistically, the observed hyperpermeability in vitro is related to disruption of the GCX components sialic acid, HS, and SDC1 following upregulation of sialidases as well as activity and subsequent expression of heparanase, enzymes known to contribute to the degradation of cellular GAGs and proteoglycans in the GCX. ${ }^{196,199}$

For the common influenza A virus (IAV) that has caused the Spanish flu (1918), Asian flu (1957), Hong Kong flu (1967) and bird flu (2004), multivalent binding to the host cell surface is a common strategy for infection. IAV binds to the host cell surface via its trimeric spike protein hemagglutinin, which recognizes sialic acids on the GCX of cellular membranes. Despite the low binding affinity of individual hemagglutinin receptor binding sites to sialic acid, a stable adhesion originates from multiple ligand-receptor binding events between the virus and the sialic acid glycoconjugates on the cell surface. ${ }^{105}$

\section{Human Corona Viruses}

(HCoV-NL63) is an alpha coronavirus that was first identified in 2004. Previous studies showed that HCoVNL63 and severe acute respiratory syndrome (SARS)CoV (2002) employ the same receptor for host cell entry, angiotensin converting enzyme 2 (ACE2), but it is largely unclear whether ACE2 interactions are sufficient to allow HCoV-NL63 binding to cells. Milewska et al. ${ }^{136}$ showed that directed expression of ACE2 on cells previously resistant to HCoV-NL63 renders them susceptible, showing that ACE2 protein acts as a functional receptor and that its expression is required for infection. However, competition experiments using a range of soluble elements of cellular membrane-as-

\section{SARS-CoV}

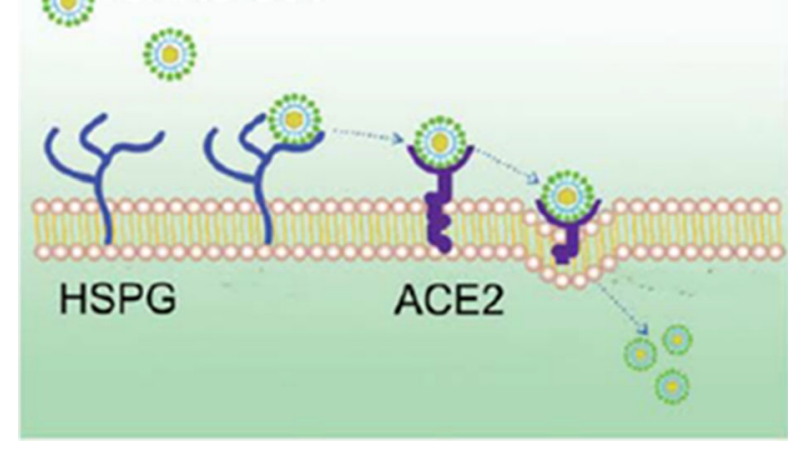

FIGURE 14. HSPGs play an important role in the process of SARS-CoV cell entry. The anchoring sites provided by HSPGs permit initial contact between SARS-CoV and host cells and the concentration of virus particles on cell surface. SARS-CoV rolls onto the cell membrane by binding to HSPGs and scans for specific entry receptors, which leads to subsequent cell entry. Reprinted from Ref. 103.

sociated components revealed that HS proteoglycans (HSPGs) constitute HCoV-NL63 adhesion receptors. Binding of HCoV-NL63 to heparan sulfates was required for viral attachment and infection of target cells.

Lang et al. ${ }^{103}$ showed that the efficient entry of SARS-CoV into the infected cells requires the involvement of HSPGs in concert with ACE2. Increased infectivity of SARS pseudovirus was associated with binding to HS. Elimination of cell-surface HS by heparinase or the addition of exogenous heparin reduced the ability of the virus to bind to the cell surface and increased cellular resistance to infection (Fig. 14).

In a recent review paper, Sardu et al. ${ }^{180}$ emphasized that to access host cells, SARS-CoV-2 (COVID-19) uses a surface glycoprotein known as spike to engage the ACE2 receptor for coronavirus entry. Therefore, the density of ACE2 in each tissue may correlate with the severity of the disease in that tissue. ACE2 is known to be well expressed by ECs. Hypertension, thrombosis, kidney failure, and diabetes all show strong co-morbidities with COVID-19 and are known to be associated with endothelial dysfunction, suggesting that COVID-19 is in a certain sense an endothelial disease.

Cardiac injury is a prevalent complication in severe COVID-19 patients, exacerbating the disease severity. ACE2, the key host cellular receptor of SARS-CoV-2, has been identified in multiple organs, and its cellular distribution in human heart was illuminated recently by Chen et al. ${ }^{27}$ They developed a single cell atlas of adult human heart tissue, and revealed that pericytes with high expression of ACE2 might act as the target cardiac cell of SARS-CoV-2. Pericyte injury due to 
virus infection may result in capillary EC dysfunction, inducing microvascular dysfunction.

\section{Malaria}

It has been proposed that a breakdown of the endothelial GCX plays a key role in severe malaria. In a study by Introini et al., ${ }^{85}$ the interactions between Plasmodium falciparum infected red blood cells (PfRBCs) and GCX were investigated in vitro. Impairment of the GCX was obtained by enzymatic removal of sialic acid residues, which, due to their terminal location and net negative charge, are implicated in the initial interactions with contacting cells. There was a more than two fold increase of PfRBC adhesion to ECs upon enzymatic treatment, relative to untreated ECs. As a control, no effect of enzymatic treatment on healthy red blood cell adhesion was found. These results provide evidence of the increased cytoadherence of PfRBCs to GCX impaired vascular endothelium, thus supporting the role of GCX disruption in the pathogenesis of this disease.

Biochemical assessment of the luminal surface of ECs from cerebral malaria (CM) mice revealed malaria-induced loss of SDC1, SDC4, HS and CS. ${ }^{75}$ This loss was mirrored in increases of the same components in peripheral blood samples. Corticosteroid treatment protected against CM, reduced inflammation, and prevented GCX loss. Adjunctive antithrombin-3 also prevented GCX loss and significantly reduced CM-associated mortality. Thus, GCX loss may be associated with malaria pathogenesis.

Yeo et $a .^{233}$ prospectively enrolled Indonesian inpatients ( $\geq 18$ years old) with severe (SM) or moderately-severe falciparum malaria (MSM) and healthy controls (HCs). GCX breakdown products were measured in samples of urine and plasma. SDC1 and GAGs were significantly increased in MSM relative to HC patients and even more so in SM patients. GCX degradation was positively associated with parasite biomass in MSM and SM patients and inversely associated with endothelial NO bioavailability. This study further supports the view that the GCX is protective against malaria.

\section{Cardiovascular and Related Diseases}

\section{Atherosclerosis}

Our 2016 review paper ${ }^{200}$ traced the observations of diminished vascular GCX in atheroprone locations in arteries from the study by Lewis et al. ${ }^{109}$ in the coronary arteries of White Carneau pigeons in the early 1980 s, through the study by van den Berg et al. ${ }^{212}$ 20 years later showed that GCX thickness was significantly reduced in the disease-prone sinus region of the mouse internal carotid artery compared to non-diseased regions in the common carotid artery. Subsequently, van den Berg et al. ${ }^{213}$ observed that intimal accumulation of low density lipoprotein (LDL) was enhanced in the internal carotid branch of mice where the GCX was thinner than in adjacent regions of the common carotid. It was suggested that the enhanced LDL accumulation was due to impaired GCX barrier properties. In a related study, Nagy et al. ${ }^{148}$ observed that inhibition of HA synthesis in a mouse model facilitated leucocyte adhesion, subsequent inflammation and progression of atherosclerosis.

More recently, Cancel et al. ${ }^{22}$ examined the GCX in the brachiocephalic artery bifurcation of $\mathrm{APOE}^{-/-}$ mice fed a high cholesterol diet for 10 weeks and observed a greatly diminished GCX on the intact ECs on plaque surfaces of the outer wall compared to the plaque free opposite wall and plaque-free descending aorta. The plaque surface was also shown to be a region of elevated EC apoptosis compared to the nonplaque regions. LDL accumulation was enhanced where the endothelial GCX was thinner and apoptosis higher than in adjacent regions of the common carotid. It was suggested that in concert with endothelial apoptosis that increases lipid permeability, glycocalyx shedding initiated by inflammation facilitates monocyte adhesion and macrophage infiltration that promote lipid retention and the development of atherosclerotic plaques.

Recent studies from H. Jo's group ${ }^{192}$ using an $\mathrm{APOE}^{-/-}$mouse model with partial ligation of the left carotid artery showed rapid development of atherosclerotic plaques ( 2 weeks) due to a mechanism involving oscillatory shear-induced upregulation of microRNA 712 that suppresses tissue inhibitor of metalloproteinase 3 . This allowed enhanced activity of MMPs that are known to degrade the GCX. ${ }^{235} \mathrm{We}$ have previously stained samples from these partial ligation experiments ${ }^{192}$ and observed a complete removal of HA in the atherosclerotic vessels. ${ }^{200}$

Recent findings indicate that SDC1 is elevated in serum, indicative of the degradation of GCX, in patients with ischemic heart disease or heart failure. ${ }^{94}$ GCX shedding markers have been examined in the pathophysiology of heart failure with reduced ejection fraction (HFrEF). HA levels and SDC1 levels were measured in HFrEF patients. HA was significantly elevated in HFrEF patients and an independent predictor for worse clinical outcome, whereas SDC1 elevation was not significant. ${ }^{152}$

Recent work with 4- to 8-week-old apolipoprotein E/low-density lipoprotein receptor deficient mice, at the stage before development of atherosclerotic plaques, displayed increased endothelial stiffness, reduced GCX coverage and thickness, reduced NO production 
in the aorta, impaired acetylcholine-induced vasodilation, and increased endothelial permeability. ${ }^{9}$ These observations are consistent with independent studies reviewed in the sections on mechanotransduction and sepsis.

Harding et al. ${ }^{74}$ using cultured EC in vitro and a mouse model, have demonstrated that pro-atherosclerotic endothelial dysfunction occurs as a result of disturbed flow-induced reduction in GCX expression and impaired endothelial sensitivity to flow. Results confirmed that disturbed flow down-regulates caveolin-1 mechanosignaling, as indicated by its reduced colocalization with phosphorylated eNOS (peNOS-serine1177), a vasoregulatory signaling molecule producing NO that is activated by shear stress through the GCX. In the absence of HS, uniform flow-conditioned ECs exhibited disturbed flow-like caveolin-1 expression, localization, and colocalization with peNOS.

\section{Stroke}

Ueno et al. ${ }^{210}$ characterized the GCX in capillaries of the frontal cortex and hypothalamus of strokeprone spontaneously hypertensive (SHRSP) rats. They observed decreased GCX abundance in the hypothalamus and frontal cortex of SHRSP rats compared to control Wistar Kyoto (WKY) rats. Similar observations of decreased GCX abundance were made in the hippocampus of SHRSP compared to WKY rats. ${ }^{210}$

The GCX is partially accessible to flowing red blood cells at its luminal side in the so called "perfused boundary region" (PBR). GCX damage results in increased PBR, which can be measured in the sublingual microvasculature. Martens et al. ${ }^{94}$ tested whether PBR is increased in patients with lacunar stroke, and further distinguished patients with white matter lesions as a sign of extensive cerebral small vessel disease. They observed that white matter lesions are associated with an increase in the red blood cell permeable part of the sublingual microvascular GCX, and this implicates a compromised GCX.

Elevated systemic haematocrit increases the risk of stroke and myocardial infarction. Richter et al. ${ }^{175}$ used an erythropoietin overexpressing transgenic mouse line (tg6) with a haematocrit of 0.85 to assess the effects of elevated haematocrit and observed that the GCX is nearly abolished in $\operatorname{tg} 6$ mice. This suggests that the pathological effects of elevated haematocrit in these mice, and possibly in polycythemic humans, relates to a reduced GCX thickness and the consequent alteration in the blood-endothelium interface.

Sehba et al. ${ }^{186}$ developed a rat model to study the mechanisms underlying subarachnoid haemorrhage. This model was used to examine the fate of microvascular GCX in the brain $1 \mathrm{~h}$ after the imposed haemorrhage. It was shown that there is a robust GCX as characterized by HA staining in the control vessels that is completely degraded in the subarachnoid haemorrhage model. ${ }^{200}$

Fihlo et al. ${ }^{206}$ emphasized that the GCX is an integral constituent of the endothelial barrier, and an increasing number of studies suggest that recovery and stabilization of the microcirculation, particularly the damaged GCX, is a major element of a successful resuscitation regimen for haemorrhagic shock. ${ }^{207}$

\section{Chronic Venous Disease}

Ligi et al. ${ }^{114}$ recently reviewed the role of the GCX in chronic venous disorders underlying common vascular pathologies - varicose veins, edema, venous ulcerations- of great medical and socioeconomic impact. The predominant pathophysiological mechanisms of chronic venous disease (CVD) start from the development of venous hypertension and reflux, leading to endothelial dysfunction, GCX degradation and venous wall dilatation. The altered hemodynamics (disturbed flow) and loss of GCX lead to altered mechanotransduction upregulating harmful biomolecular pathways, creating a vicious cycle among shear stress, proteolytic remodeling, and inflammatory processes.

\section{Hypertension and Aging}

In our previous review ${ }^{22}$ we described studies showing a loss of GCX in the blood-brain barrier of spontaneously hypertensive rats, ${ }^{210,211}$ the retinal and choroidal capillaries of rats with diabetes and hypertension, ${ }^{99}$ as well as reduced GCX thickness associated with elevated salt concentration in blood that reduces the salt sequestering capacity of the GCX leading to salt overload and hypertension. ${ }^{100,155}$ It was also shown that RBCs from hypertensive rats are subject to enhanced GCX cleavage compared to the RBCs of the normotensive rats due to the action of blood-borne MMPs. ${ }^{155}$

More recently, Schierke et al. ${ }^{181}$ have shown that high plasma $\mathrm{Na}+$ concentration associated with hypertension stiffens the endothelial cortex and diminishes the GCX leading to decreased NO while inducing endothelial release of pro-inflammatory cytokines promoting monocyte adhesion. Expression of GCX markers in plasma, including HS, HA, and SDC1, was detected in monocrotaline-induced pulmonary artery hypertension in rats indicative of endothelial GCX shedding. ${ }^{66}$ Endothelial GCX is impaired in untreated hypertensive patients and is related to arterial stiffness and coronary, and myocardial dysfunction. ${ }^{84}$ Preeclampsia related hypertension also 
leads to GCX degradation leading to endothelial dysfunction, reduced microvascular perfusion and vascular injury. ${ }^{222}$

In a study of the GCX in microvessels of young and old male mice using intravital microscopy and transmission electron microscopy and in human participants using intravital microscopy, GCX thickness in mesenteric and skeletal muscle microvessels was 51$54 \%$ lower in old compared with young mice. A $33 \%$ lower GCX thickness in the sublingual microcirculation of humans was observed in advanced age. These data provide evidence that a diminished GCX is present in advanced age and is accompanied by markers of impaired microvascular perfusion. ${ }^{119}$

\section{Alzheimers and Dementia}

Nagga et al. ${ }^{147}$ have demonstrated changes in the levels of HA in cerebrospinal fluid (CSF) in patients with Alzheimer's disease (AD) with documented vascular alterations. To further investigate if the level of $\mathrm{HA}$ in CSF can be used as a clinical diagnostic biomarker to identify vascular pathology, a study of patients with vascular dementia $(\mathrm{VaD})(\mathrm{n}=46)$, $\mathrm{AD}$ $(n=45)$, and controls without dementia $(n=26)$ was undertaken. They found significantly increased levels of HA in CSF from patients with VaD compared with controls, whereas the levels of HA in patients with $\mathrm{AD}$ were found to be unaltered compared with controls and patients with $\mathrm{VaD}$. They also found a significant positive correlation between the levels of HA and the $\mathrm{CSF} /$ serum albumin ratio, an indicator of blood-brain barrier integrity, in patients with $\mathrm{VaD}$ and $\mathrm{AD}$, supporting the role of HA in vascular changes in the brain.

\section{Diabetes}

Hyperglycaemia that is linked with diabetes has been shown in vitro to lead to loss of HS and associated shear stress-induced phosphorylation of $\operatorname{eNOS}^{116}$ and elongation of ECs in the direction of shear ${ }^{17}$ that are caused by mechanotransduction mediated by HS. In diabetic individuals, it has been observed that atherosclerosis is distributed more uniformly in arteries, not following the typical association with regions of low shear or disturbed flow near bifurcations and curvatures. ${ }^{40}$ In addition, vasodilation is altered in diabetes. ${ }^{174}$ All of these observations imply a fundamental difference in mechanotransduction by the ECs of diabetic individuals that may be explained in part by alterations in the GCX.

Endothelial dysfunction and increased risk for cardiovascular morbidity and mortality are associated with renal failure. Vlahu et al. ${ }^{216}$ demonstrated that dialysis patients shed HA and SDC1 into their blood and had an impaired GCX barrier. Salmon et al. ${ }^{179}$ reviewed the role of dysfunction of the GCX in the glomerulus leading to albuminuria and systemic increases in microvascular permeability.

Padberg et al. ${ }^{158}$ examined whether renal failure correlated with structural changes in the GCX using nephrectomized rats and patients with chronic kidney disease (CKD). The physical properties of the GCX were studied in rats using AFM. The results showed that CKD is associated with a damaged GCX and that increased GCX breakdown products correlate with the physical stability and thickness of the GCX.

Further evidence that HSPGs are shed in diabetes was provided by Ramnath et al. ${ }^{173}$ They demonstrated that the inflammatory cytokine TNF- $\alpha$ which induces disruption of the glomerular GCX in diabetes, reduced cell surface SDC4 and HS significantly. The mechanism involved MMP 9-mediated shedding of SDC4/ HS. In addition, Neves et al. ${ }^{150}$ evaluated the relationship between plasma SDC1 levels and renal dysfunction in patients with acute decompensated heart failure. They determined that plasma SDC1 levels predicted increased risk of developing acute kidney injury.

Patients with type 1 diabetes present increased plasma levels of both HA and hyaluronidase 1 (HYAL1) that degrades HA. Dogne et al. ${ }^{44,45}$ investigated the role of HYAL1 in the development of diabetes-induced endothelial dysfunction, endothelialdependent vasodilation, arteriolar GCX size, and glomerular barrier properties in wild-type and HYAL1 knockout mice with or without streptozotocin (STZ)induced diabetes. Four weeks after STZ injections, the lack of HYAL1 prevented diabetes-induced increases in soluble P-selectin concentrations, limited the impact of the disease on vasorelaxation, increased GCX thickness, maintained GCX structure and HA content during diabetes, and prevented diabetes-induced glomerular barrier dysfunction-all suggesting that HYAL1 contributes to endothelial and GCX dysfunction induced by diabetes.

Leskova et al. ${ }^{106}$ investigated the effects of diabetes and HYAL1 on the thickness of the GCX in the mouse retina. The retinal circulation of diabetic Ins2 (Akita) mice and their nondiabetic littermates were observed via intravital microscopy. In nondiabetic C57BL/6 mice, the GCX thickness also was evaluated prior to and following infusion of HYAL1. Both diabetes and hyaluronidase infusion significantly reduced the thickness of the GCX in retinal arterioles (but not in venules), and hyaluronidase increased retinal microvascular leakage. The findings demonstrate that diabetes and hyaluronidase reduce the thickness of the retinal GCX, in which HA plays a significant role in barrier function. 
The mechanical properties of the GCX and endothelium in ex vivo mouse aortas were determined simultaneously in indentation experiments with AFM for diabetic and control mice at ages of 1119 weeks. $^{203}$ In diabetic mice, marked endothelial stiffening and reduced GCX coverage were present already in 11-week-old mice and persisted in older animals. In contrast, reduction of GCX length was progressive and was most pronounced in 19-week-old diabetic mice. The reduction of GCX length correlated with an increasing level of glycated haemoglobin and decreased endothelial NO production secondary to loss of mechanotransduction through the GCX. The stiffening of ECs and diminished GCX coverage occurred in early diabetes and were followed by the reduction of the GCX length that correlated with diabetes progression.

\section{Exercise and Endurance Training}

A recent study evaluated whether oxidative stress and inflammation induced by strenuous exercise affect GCX integrity and endothelial function in healthy young men. No significant changes in serum SDC1 and HS concentrations were observed, and it was concluded that a single bout of severe-intensity exercise is well accommodated by endothelium in young, healthy men as it neither results in evident GCX disruption nor in the impairment of $\mathrm{NO}$ or prostacyclin production. ${ }^{121}$ In a follow up study, Majerczak et al. ${ }^{122}$ found that moderate-intensity endurance training exerts a pronounced protective effect on endothelial GCX integrity at rest and during exercise. Training led to a significant decrease in basal and end-exercise concentrations of blood markers of GCX damage (SDC1 and HS). They suggested this was the result of an improvement of antioxidant defense that may represent the vasoprotective mechanism highly responsive to moderate-intensity training.

High-intensity interval training (HIIT) has been proposed to exert vasculoprotective effects. A study by Schmitz et al. ${ }^{183}$ evaluated whether HIIT affects the microvasculature, including the endothelial GCX barrier. Sidestream dark-field imaging was performed at baseline and follow-up to detect changes of the sublingual microvasculature including the endothelial GCX thickness. At baseline, a significant correlation between maximal exercise performance capacity and glycocalyx thickness was detected. Increased exercise performance at follow-up also correlated with GCX thickness. Moreover, increased baseline microRNA143 (miR-143) levels predicted increased GCX thickness at follow-up. miR-143 may represent a tool for monitoring early vasculoprotective adaptations to physical activity.

\section{Trauma}

Endothelial dysfunction has emerged as a critical component associated with hemorrhagic shock and trauma. ${ }^{90}$ The endothelial GCX is an integral constituent of the endothelial protective barrier, and an increasing number of studies suggest that recovery and stabilization of the microcirculation is associated with GCX recovery. 206,207

Johansson et al. ${ }^{90}$ have reported in trauma patients, that among the 17 markers of GCX degradation and endothelial damage, high circulating SDC1 was associated with increased mortality in patients with penetrating injury and severe head injury. Chignalia et al. ${ }^{28}$ have reviewed the GCX in lung injury and emphasized that the respiratory epithelial GCX is a target in bacterial pneumonia with emphasis on SDC1 and hyaluronan.

\section{Cancer (Cancer Cell Glycocalyx)}

The cancer cell GCX mediates the interactions between a tumor and its microenvironment and profoundly affects cancer cell signaling, migration, invasion, and metastasis. ${ }^{18}$ Paszek et al. ${ }^{163}$ observed that circulating tumor cells (CTCs) from patients with aggressive breast cancers overexpressed bulky glycoproteins. To test whether bulky glycoproteins could facilitate survival at distant sites, they incorporated a long glycoprotein mimetic into non-malignant mammary epithelial cells and found cells remained viable when plated on soft hydrogels that mimic sites of colonization, whereas untreated cells were not viable. The bulky GCX promoted growth and survival by increasing integrin adhesion and signaling. Woods et al. $^{224}$ coated tumor cells with synthetic glycopolymers of various thicknesses and found that longer, bulkier GCX promoted cell survival and proliferation thus increasing their metastatic potential in a mouse model.

Altered expression of the GAG components of the cancer cell GCX, and their cell surface core proteins and receptors, has long been linked to cancer progression. Increased HA expression is consistently found in many types of cancers. Increases in tumor cell associated HA is seen in tumors arising from simple epithelia such as ovarian, colorectal, and gastric cancer, while increases in HA expression in the tumor stroma are observed in, for example, breast, prostate, and bladder carcinomas, and lung adenocarcinomas. In either case, increased HA expression is associated with tumor progression and poor survival. ${ }^{198}$ HA interactions with its cell surface receptors activate signaling pathways that modulate cell motility, proliferation, differentiation, and invasiveness. ${ }^{92,139}$ 
CD44 is the major cell surface receptor for HA. Due to alternative splicing and post-translational modifications, multiple variants are generated $(\mathrm{CD} 44 \mathrm{v})$. While the standard form of CD44 (CD44s) is expressed in most cells, CD44v are expressed in tumor cells and have been shown to promote tumor progression and metastatic potential in lung, breast, and colon cancer. ${ }^{139}$ Another HA receptor, RHAMM, is upregulated in various cancers and its expression is associated with poor prognosis in lung, breast, colorectal, gastric, pancreatic, and ovarian cancers. ${ }^{26}$ The role of HA-CD44 and HA-RHAMM interactions in cancer has been reviewed in detail. ${ }^{138}$

HS interacts with a wide range of growth factors and chemokines making it essential to many aspects of cell regulation and function. ${ }^{62}$ Modification of HS by enzymes such as heparanase, sulf-1, and sulf-2 plays an important role in regulating cancer growth ${ }^{35}$ and altered expression of these enzymes is found in many types of cancers. ${ }^{71,190}$ Jung et al. ${ }^{91}$ found that heparanase degrades HS on SDC1 exposing a binding site that couples vascular endothelial growth factor receptor- 2 to $\alpha 4 \beta 1$ integrin in multiple myeloma, leading to tumor cell migration and invasion. Shinyo et al. ${ }^{189}$ found reduced expression of HS was associated with the progression of cervical intraepithelial neoplasms to invasive cancer. Through a different mechanism, HS on the cancer cell surface mediates flow-induced migration and metastasis in renal carcinoma cells (discussed below). ${ }^{169,170}$ Thus the role of HS in tumor progression may be context and cell type specific.

The syndecan and glypican proteoglycans interact, directly or through their HS chains, with an array of extracellular matrix proteins that mediate cell-signaling events during carcinogenesis ${ }^{86}$ (see Table 1). As with HS, the role of SDC1 may depend on cell type and disease stage. Loss of SDC1 appears to be a key step in regulating epithelial to mesenchymal transition. ${ }^{86} \mathrm{Re}-$ cently, Kind et al. ${ }^{95}$ examined tissue microarrays for $\mathrm{SDC1}$ expression and found it was highest in squamous cell carcinomas such as esophagus, cervix, and lung and in adenocarcinomas in lung and colorectal cancer. Expression was low in germ cell tumors, sarcomas, endocrine, and neuroendocrine tumors. Barbareschi et $a l .{ }^{10}$ reported that SDC1 was highly expressed and associated with aggressive phenotype in breast carcinoma. By contrast, SDC1 was downregulated in invasive cervical cancer ${ }^{189}$ and multiple myeloma. ${ }^{91}$

Syndecan-2 plays an important role in mediating cell shape, adhesion and migration. ${ }^{86}$ Syndecan-2 overexpression is found in low metastatic clones of Lewis lung carcinoma, and upregulation of syndecan-2 in highly metastatic clones inhibits their metastasis. ${ }^{191}$ In colon ${ }^{31,162}$ and breast ${ }^{115}$ carcinoma cell lines, syndecan-2 promotes tumor progression by mediating cell adhesion and enhancing migration. Shed syndencan-2 inhibits angiogenesis which affects tumor growth. ${ }^{42}$

SDC4 is expressed in most tumors and mediates cell adhesion to the extracellular matrix. SCD4 is downregulated in invasive colon carcinoma ${ }^{86}$ but upregulated in the renal cell carcinoma tissue samples. ${ }^{52}$ The specific roles SDC4 plays in tumor progression and metastasis may depend on cell type and other factors in the tumor microenvironment ${ }^{86}$ and more studies are needed to advance our knowledge in this area.

High levels of GPC1 have been reported in lymphoma, ${ }^{54}$ breast, ${ }^{126}$ pancreatic, ${ }^{46,97,117}$ esophageal, ${ }^{72}$ and cervical cancer. ${ }^{127}$ In breast and pancreatic cancer, GPC1 was shown to modulate the mitogenic response of cancer cells to heparin-binding growth factors. ${ }^{97,126}$ GPC1 expression was also a predictor of perineural

TABLE 1. Syndecans and glypicans in cancer.

\begin{tabular}{lll}
\hline Component & \multicolumn{1}{c}{ Expression in cancer/possible mechanisms } & References \\
\hline $\begin{array}{l}\text { Syndecans } \\
\text { Syndecan-1 }\end{array}$ & $\begin{array}{l}\text { Upregulated in squamous cell carcinomas, aggressive breast carcinomas; downregulated in } \\
\text { sarcomas, germ cell, and endocrine tumors. Regulates epithelial to mesenchymal transition }\end{array}$ & $10,86,91,95,189$ \\
Syndecan-2 & $\begin{array}{l}\text { Upregulated in highly metastatic Lewis lung carcinoma; promotes tumor progression in breast and } \\
\text { colon carcinomas }\end{array}$ & $31,42,86,115,162,191$ \\
Syndecan-4 & $\begin{array}{l}\text { Upregulated in renal cell carcinoma; downregulated in invasive colon carcinoma. Mediates cell } \\
\text { adhesion to extracellular matrix }\end{array}$ & 52,86 \\
Glypicans & Upregulated in lymphoma, breast, pancreatic, and cervical cancer. Modulates mitogenic response & $19,46,54,72,97,110,111$, \\
Glypican-1 & & $\begin{array}{l}117,126,127,131,166, \\
177,178,218\end{array}$ \\
Glypican-3 & $\begin{array}{l}\text { Upregulated in hepatocellular, gastric, ovarian and lung squamous cell carcinoma; downregulated } \\
\text { in breast, lung, ovary, and mesothelium cancers }\end{array}$ & $67,86,153$ \\
\hline
\end{tabular}


invasion $^{46}$ and poor prognosis ${ }^{117}$ in patients with pancreatic cancer. Knockdown of GPC1 in human colon carcinoma cell lines resulted in a decrease in invasion and migration. ${ }^{111}$ In patients with glioblastoma, elevated GPC1 expression was correlated with tumor dissemination and shorter overall survival. ${ }^{178}$ The use of GPC1 antibodies as a diagnostic tool to detect circulating tumor cells and exosomes, as well as labeling of cancer cells in situ, is an active area of investigation. ${ }^{19,110,131,166,177,218}$

Glypican-3 is found to be downregulated in breast, lung, ovary, and mesothelium cancers. ${ }^{86}$ By contrast, glypican-3 is overexpressed in hepatocellular, gastric, ovarian, and lung squamous cell carcinomas, and melanomas. ${ }^{153}$ In hepatocellular carcinoma, glypican-3 is actively investigated as a tool for diagnosis and treatment. ${ }^{67}$

\section{Cancer Cell Mechanotransduction}

Interstitial flow (IF) is enhanced in tumors due to the leaky nature of tumor vasculature and increased interstitial pressure in the tumor microenvironment. ${ }^{146}$ This enhanced flow has been shown to alter the migration and invasiveness of several types of cancers. $^{70,82,145,169,171,188}$ The role of the GCX as a mechanosensor for IF has only recently begun to be investigated. Our previous review ${ }^{200}$ looked at the in vitro work of Qazi et al. ${ }^{169}$ showing IF significantly increased migration of a highly metastatic kidney cell line (SN12L1). The same levels of flow did not increase migration in a low metastatic kidney carcinoma cell line (SN12C). The effect was completely inhibited by removal of either HS with heparinase or HA with hyaluronidase. This work was recently extended to an animal model to investigate the possibility that cancer cell GCX components mediate metastasis. Qazi et al. ${ }^{170}$ implanted SN12L1 cells in the renal capsule of mice and assessed metastatic colonization to distant organs. They used shRNA to knockdown NDST1 in SN12L1 cells thus suppressing synthesis of HS. This resulted in a $95 \%$ reduction in metastasis of tumors formed by knockdown cells compared to tumors formed by unmodified cells (wild type). In addition, they found that HS deficient tumors had well-defined boundaries and did not invade the surrounding normal tissue, while wild type tumors had poorly defined margins and aggressively invaded the surrounding tissue.

Kingsmore et al. ${ }^{96}$ used patient-derived xenograft models to determine the effect of high flow on invasiveness of glioblastoma stem cells. They observed increased invasion into high flow regions compared to low flow regions in two of the three patient-derived cell lines. Examining the cell lines in vitro, they found that the flow response could be occurring through two mechanisms: autologous chemotaxis mediated by CXCR4/CXCL2, or mechanotransduction mediated by $\mathrm{CD} 44$. Characterization of each cell line revealed different percentages of CXCR4, CXCL2, and CD44 expressing cells indicating that subpopulations of glioblastoma stem cells could respond to flow via either mechanism within a single patient.

Moran et al. ${ }^{142}$ investigated the specific proteins that link surface GAGs that sense IF to the cancer cell body where mechanotransduction resulting in increased migration occurs. They measured the expression of GPC1, SDC1, and CD44 in high (SN12L1) and low (SN12C) metastatic kidney cell lines and found significantly higher GPC1 levels in the high metastatic cell line. Knockdown of GPC1 by siRNA resulted in significant inhibition of flow-induced migration in the SN12L1 cell line. While the baseline (static) protein expression of CD44 was not different between SN12L1 and SN12C cells, this group has previously shown that $4 \mathrm{~h}$ of flow significantly increased CD44 gene expression in SN12L1 cells and that blocking CD44 expression resulted in blocking flow-induced migration. ${ }^{169}$ Recently, they investigated the possibility that the other HA surface receptor, RHAMM, could also act as the mechanical link between HA and the cell. They found increased levels of RHAMM in SN12L1 compared to SN12C, and knockdown of RHAMM by siRNA significantly reduced flow-induced migration in the SN12L1 cell line (unpublished data). A recent study by Mele et al. ${ }^{130}$ found that RHAMM knockdown slowed tumor growth and inhibited metastasis of colorectal cancer cells in a mouse model.

In summary, differential expression and modification of GCX components appear to play key roles in cancer progression. Cell-associated and extracellular matrix HA and its cell-surface receptors CD44 and RHAMM are upregulated in most cancers and are associated with poor prognosis. Heparanase mediated shedding of HS on SDC1 enables growth factor in the tumor microenvironment to access receptors and activate cell signaling that fosters tumor growth and progression. On the other hand, HS on GPC1 acts as a mechanosensor for IF that alters migration and invasiveness in some types of cancer. Finally, abnormal glycosylation ${ }^{18,143}$ and GAG modification by enzymes $^{35,71}$ (e.g. Heparanase, sulf-1) are actively investigated as key mechanisms in tumor growth and metastasis.

\section{Cancer Metastasis (Endothelial Cell Glycocalyx)}

The major cancer metastatic steps from a solid tumor include migration of tumor cells from the 
Control
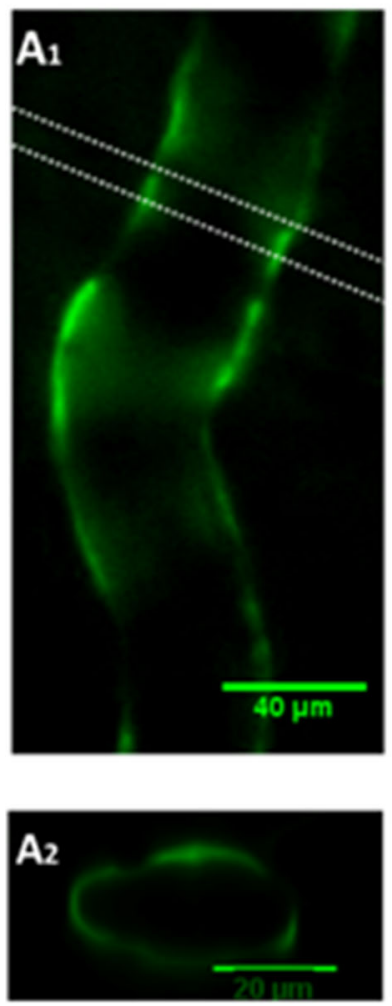

Initial Adhesion
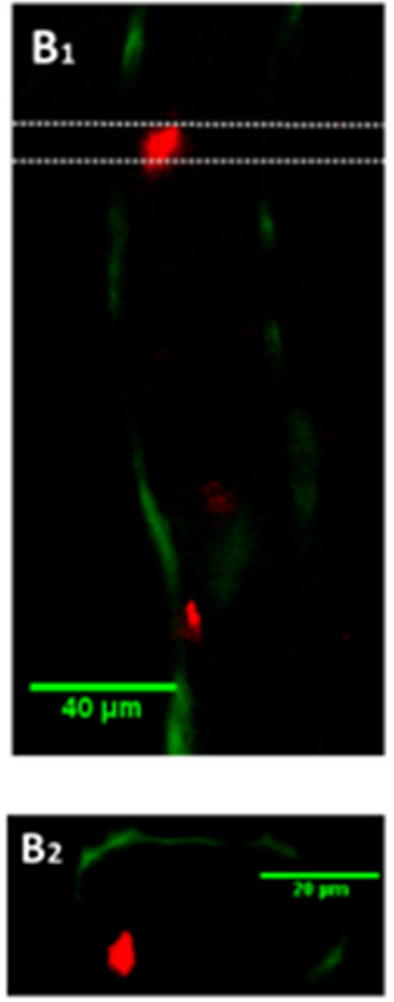

45 min Perfusion
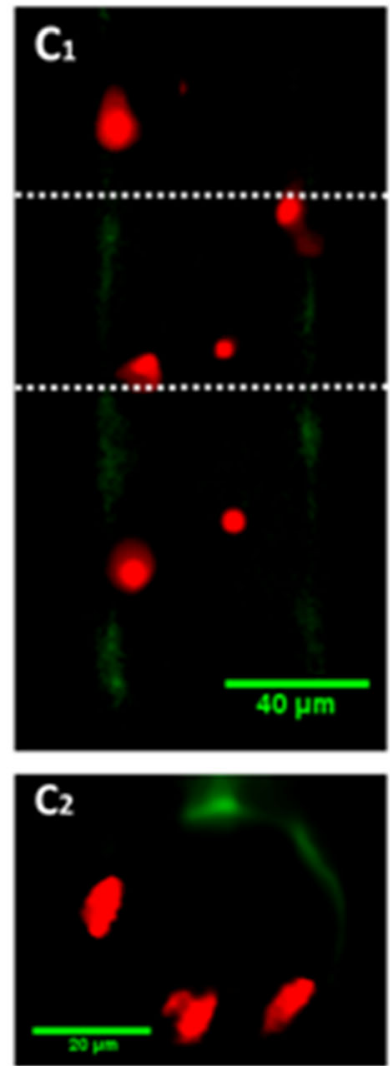

FIGURE 15. Endothelial surface glycocalyx labeled with FITC-anti-HS (green) on a rat mesenteric microvessel (left panel) without breast cancer cell adhesion (left panels), at initial adhesion (middle panel, red spots are cancer cells) and after $\mathbf{4 5}$ min perfusion (right panel). Reprinted from Ref. 21.

TABLE 2. Drugs that rescue the GCX.

\begin{tabular}{lll}
\hline Compound & \multicolumn{1}{c}{ Possible mechanisms } & References \\
\hline Albumin/S1P & Regenerate GCX, inhibit shedding & $4,11,132,235,238$ \\
Sulodexide & Supplement GCX, inhibit degrading enzymes & $30,112,123,193,194,214$ \\
Rhamnan Sulfate & Enhances HS synthesis & $23,156,197$ \\
Sevoflurane & Inhibit shedding, increase GCX regeneration & $5,24,93,113$ \\
Biomimetic Nanoparticles & Replace shed GCX & 223,243 \\
ALM BHB/M P-188 & Inhibit shedding & 206 \\
Blueberry Metabolites & Regenerate GCX & $13,16,38$ \\
Empagliflozin & Regenerate GCX & 34,83 \\
Metformin & Regenerate GCX & $8,53,102,204,214$ \\
\hline
\end{tabular}

S1P sphingosine-1 phosphate, ALM adenosine + lidocaine $+\mathrm{Mg}, B H B / M$ beta-hydroxybutryrate + melatonin, $P$-188 poloxamer 188 .

original site to nearby microvessels, intravasation into the blood circulation through the microvessel wall, arrest and adhesion to the microvasculature at a distant organ, interaction with the ECs forming the microvessel wall and extravasation from the leaky endothelial barrier to the surrounding tissue, survival, proliferation and formation of a new colony. While cancer cell GCX enhances cancer cell migration in the tissue during its metastasis, damage of endothelial GCX has been found during tumor cell adhesion and extravasation. An in vivo study by Cai et al. ${ }^{21}$ showed that adhesion of malignant breast cancer cell MDAMB-231 to microvessel walls degrades endothelial GCX in a timely manner (Fig. 15). Preservation of the 
GCX either by a plasma glycoprotein orosomucoid ${ }^{21}$ or sphingosine-1-phosphate (S1P), ${ }^{245}$ and inhibition of VEGF receptor (KDR/Flk-1) ${ }^{187}$ can reduce the tumor cell adhesion. In vitro studies also found that breast cancer cell adhesion to and transmigration across an in vitro blood-brain barrier significantly destroy the endothelial GCX but not the cancer cell GCX. ${ }^{56}$ Although the mechanism by which cancer cells degrade the endothelial GCX is not completely understood, integrin $\beta 4$ signaling ${ }^{65}$ enhanced ErbB2-dependent expression of VEGF by tumor cells ${ }^{55}$ is a likely reason. VEGF can degrade $\mathrm{GCX}^{21,56}$ to expose more cell and extracellular matrix adhesion molecules, e.g. E-selectin, ${ }^{172}$ at the endothelium and underneath the basement membrane, e.g. laminin, ${ }^{55}$ for tumor cell adhesion and transmigration.

\section{Drugs and Strategies to Rescue the Glycocalyx}

A previous review ${ }^{200}$ summarized a number of emerging approaches to rescue and/or stabilize the GCX. Below we update with recent developments on these and newer approaches. These are summarized in Table 2. Save for a few exceptions, the effects of the drugs discussed below on the GCX have not yet been tested in human trials and the results derive from in vitro and/or animal studies.

\section{Albumin $/ S 1 P$}

The administration of plasma (albumin) is an established practice in the treatment of massive hemorrhage and trauma. A recent review summarizes the role of plasma in protecting the endothelium. ${ }^{11}$ A key mediator in the effect of plasma on the endothelial GCX may be albumin-bound S1P, which has been shown to inhibit the shedding of SDC1 and regenerate the GCX. ${ }^{235,238}$ Recently, Mensah et al. ${ }^{132}$ combined S1P with exogenous HS to restore expression of Connexin 43 and interendothelial communication after GCX degradation with heparinese III in vitro. While both S1P and exogenous HS restored HS after degradation, only the combination of both agents was found to restore interendothelial communication. Alves et al. ${ }^{4}$ used an in vivo rat model of hemorrhagic shock and resuscitation (HSR) to show that S1P reduces microvascular leakage induced by HSR and protects against GCX degradation. In vitro, they also show that S1P protects against mitochondrial dysfunction-induced endothelial barrier dysfunction by acting through mitochondrial complex III.

\section{Sulodexide}

Sulodexide is a highly purified GAG extracted from the porcine intestinal mucosa. It is composed of $80 \%$ fast-moving heparin (a HS mimetic) and 20\% dermatan sulfate and is available for oral, intramuscular, and intravenous administration. Sulodexide has been applied in clinical trials for the treatment of CVD and diabetic nephropathy. ${ }^{30}$ Song and Goligorsky ${ }^{193}$ hypothesize that the superior effects of sulodexide are due to its dual action: supplementation of GCX components, and inhibition of GCX degrading enzymes. Fast-moving heparin inhibits heparanase while dermatan sulfate blocks the pro-MMP-9 molecule from changing into the active form.

The effect of sulodexide on endothelial function was recently assessed by Li et al. ${ }^{112}$ in the balloon-injury carotid artery model. Electron microscopy showed that sulodexide reconstituted the GCX structure thus preserving endothelial function. The authors found that sulodexide treatment increased eNOS levels, reduced endothelial hyperplasia, inhibited platelet aggregation, decreased expression of CD31, ICAM-1, and VCAM1 , and reduced the infiltration of CD68-positive cells.

Van Haare et al. ${ }^{214}$ studied the effects of a 4-week sulodexide treatment on microvascular perfusion capacity of high fat diet-fed rats. They showed that sulodexide restored the hyperemic response to adenosine in myocardial perfusion, which was blunted by the high fat diet in control animals.

Degradation of the GCX is an early event in sepsis which contributes to high mortality. Song et al. ${ }^{194}$ used sulodexide to accelerate GCX restoration which resulted in reduced vascular permeability and improved animal survival in a mouse model of sepsis.

CVD patients treated with sulodexide show significant improvements in peripheral venous pressure, patient-assessed quality of life scores, and ulcer healing, along with reductions plasma concentrations of MMP9, IL-6, and MCP-1, and reductions in other clinical signs and symptoms. ${ }^{123}$

\section{Rhamnan Sulfate}

Suzuki and Terasawa ${ }^{197}$ recently reviewed the many beneficial biological activities of rhamnan sulfate (RS), a sulfated polysaccharide extracted from the green algae $M$. nitidum. RS is found to have antiviral and antithrombotic activities. It mitigates the high-fat diet induced elevation of LDL cholesterol, and the postprandial increase in blood glucose level in healthy human subjects. RS also has anti-inflammatory effects on endothelial cells: it suppresses the elevation of tissue factor and $\mathrm{vWF}$ in TNF- $\alpha$ or thrombin-induced inflammation of ECs. ${ }^{156}$ We have previously shown that RS enhances the GCX and reduces LDL permeability of cultured ECs. ${ }^{23}$ Recently, Aaron Baker's lab studied the effects of oral rhamnan sulfate on a mouse model of atherosclerosis and found significant reduc- 
tions in vascular inflammation and atherosclerotic plaque formation (unpublished data).

\section{Sevoflurane}

Sevoflurane, a volatile anesthetic, has been shown to protect the GCX during ischemia and reperfusion (IR). Annecke et al. $^{5}$ first showed that ischemia led to shedding of HS and SDC1, and degradation of the GCX in isolated guinea pig hearts. Treatment with sevoflurane before or after IR was found to attenuate shedding of HS and SDC1. Treatment pre- plus postIR with sevoflurane was found to also preserve the GCX structure. Chappell et al. ${ }^{24}$ showed sevoflurane pretreatment also reduced leukocyte and platelet adhesion after IR. In a study on Sprague-Dawley rats, Li et al. ${ }^{113}$ showed sevoflurane reduced the shedding of HS and SDC1, and preserved the endothelial GCX in the liver following hepatic ischemia. The mechanisms by which sevoflurane inhibitis GCX shedding in IR are not well understood. Annecke et al. ${ }^{5}$ found that sevoflurane treatment reduced the release of cathepsin $\mathrm{B}$, a protease stored in lysosomes and associated with degradation of extracellular matrix and inflammatory diseases. A recent study on rat aortas found that sevoflurane promoted regeneration of the GCX by upregulating the sialytransferase ST6Gal-1. ${ }^{93}$

\section{Biomimetic Nanoparticles and Surfaces}

Wodicka et al. ${ }^{223}$ developed a coating composed of a dermatan sulfate backbone containing selectinbinding peptides to target endothelium with exposed adhesion molecules due to loss of the GCX. The coating was shown to reduce platelet activation and binding to ECs in vitro. In an in vivo mouse model of deep vein thrombosis the coating reduced thrombus formation without decreasing the whole blood clotting time, suggesting its potential to overcome the negative side effects of standard anticoagulant therapies such as heparin.

Liposomal nanocarriers of preassembled GCX have been developed by the Goligorsky Lab. ${ }^{243}$ The nanocarriers restored mechanically-induced NO production in cultured ECs pretreated with heparinase III. The flow-induced vasodilatation of mesenteric arteries with degraded GCX was also restored after perfusion with the nanocarriers.

\section{Others}

Because of increased recognition of the role of the GCX as a key mediator in vascular diseases, drugs and natural compounds previously shown to have beneficial properties are now being investigated for their ef- fects on the GCX. Below we summarize some recent developments.

Torres Filho et al. ${ }^{206}$ tested three drugs previously shown to improve outcomes after ischemia and hemorrhage for their effect on the GCX in a rat model of hemorrhagic shock and resuscitation. They found that all three compounds tested, adenosine + lidocaine $+\mathrm{Mg}$ (ALM), beta-hydroxybutryrate + melatonin $(\mathrm{BHB} / \mathrm{M})$, and poloxamer 188 (P-188) significantly restored the GCX thickness compared to resuscitation with lactated Ringer's solution. Lower plasma SDC1 levels were also found with all three drugs. While the mechanisms by which these drugs restore the GCX are unknown, it is suggested that they may counteract the production of reactive oxygen species and oxidative stress that leads to GCX shedding.

Blueberry metabolites have been shown to attenuate endothelial dysfunction in an in vitro model of lipoxocity, ${ }^{16}$ and clinical studies support a cardio-protective role of blueberries in individuals with metabolic syndrome. ${ }^{13}$ Recently, Cutler et $a .^{38}$ showed that blueberry metabolites restore GAGs, and reduce gene expression of inflammatory markers and monocyte binding on human aortic ECs derived from diabetic patients, suggesting that some of the beneficial effects of blueberries are due to protection and restoration of the GCX.

Empagliflozin is a sodium-glucose co-transporter-2 used to treat type 2 diabetes mellitus shown in clinical trials to significantly reduce cardiovascular events in addition to improving glycemic control. Cooper et al. ${ }^{34}$ recently showed that empagliflozin restores the GCX on human abdominal aortic ECs (HAAECs) after degradation with heparinase III. In addition, empagliflozin restored the flow-induced elongation, and inhibited leukocyte cell adhesion to HAAECs with degraded GCX. A new clinical trial ${ }^{83}$ found that treatment with empagliflozin showed greater improvement of vascular markers and endothelial GCX thickness than insulin treatment in patients with type 2 diabetes mellitus.

Metformin is the most popular anti-diabetic drug in the United States and has been shown to have vasculoprotective $^{8}$ and anticancer ${ }^{102}$ properties in type 2 diabetes patients independent of its glucose lowering effects. Eskens et al. ${ }^{53}$ subjected a mouse model of type 2 diabetes to a 2-week metformin treatment and found improved endothelial GCX barrier properties compared to the untreated group. Notably, this improvement occurred without any effect on blood glucose levels. In the same sulodexide study cited above, Van Haare et al. ${ }^{214}$ showed that metformin was as effective as sulodexide in restoring the hyperemic response to adenosine in myocardial perfusion in high-fat fed rats. 
More recently, Targosz-Korecka et al. ${ }^{204}$ found in an in vitro study that metformin treatment restores the GCX, downregulates surface expression of adhesion molecules ICAM and E-selectin, and reduces stiffness of ECs grown in high glucose. In addition, they found that treatment with metformin reduced the adhesive interaction between ECs and human lung carcinoma cells. It is suggested that both the regeneration of the endothelial GCX and downregulation of adhesion molecules may contribute to the antimetastatic effects of metformin.

\section{Drugs and Strategies to Target the Glycocalyx for Cancer Treatment}

\section{Carrier-Mediated Drug Delivery Systems}

Because many cancer cells overexpress HA receptors CD44 and RHAMM, targeting of these using the HA molecule itself has been used to deliver cytotoxic agents directly to cancer cells. For example, nimesulide is a hydrophobic COX-2 inhibitor anti-inflammatory known to also inhibit tumor growth in various types of cancer by inducing apoptosis. Jian et al. ${ }^{89}$ used an HAnimesulide conjugate to target CD44-overexpressing HT-29 colorectal cancer cells in a mouse model improving the drug's selectivity and solubility. In vitro HA-nimesulide increased apoptosis in HT-29 cells to $47.1 \%$, compared to $2.9 \%$ in untreated cells. Tumor growth was inhibited by $82.3 \%$ in mice treated with HA-nimesulide, compared to control, whereas it was inhibited by $55.9 \%$ in mice treated with free nimesulide. Yang et al. ${ }^{228}$ used HA conjugated with folic acid to form micelles that were then loaded with doxorubicin (DOX). In vitro cytotoxicity tests revealed that the DOX-loaded micelles were less toxic to tumor cells than free DOX, likely because free DOX could diffuse quickly into the cells while the micelles had to be internalized via endocytosis, delaying the release of DOX into the cells. However, when the micelles were used in nude mice bearing human liver cancer cells tumors, which express CD44, they resulted in improved targetability of DOX to the tumors. Zhong et al. ${ }^{246}$ developed HA micelles loaded with paclitaxel to successfully inhibit tumor growth of MCF-7 human breast tumor xenografts in mice.

\section{Antibody-Based Therapies}

Antibody-drug conjugates (ADCs) are a targeted therapy wherein a cytotoxic agent is linked to an antibody. A number of ADCs have been developed that target GCX components that are overexpressed in cancer cells. Matsuzaki et al. ${ }^{127}$ developed a novel antiGPC1 antibody and conjugated it to the cytotoxic agent monomethyl auristatin F (MMAF) for the treatment of uterine cervical cancer. In preclinical studies, the GPC1-ADC was internalized and was cytotoxic in HeLa and ME180, two uterine cervical cancer cell lines that overexpress GPC1, while it had no effect on an ovarian cancer cell line that did not express GPC1. The GPC1-ADC also inhibited tumor growth in a murine xenograph model. The same group developed a different GPC1-ADC, also conjugated to MMAF, which showed tumor growth inhibition in patient-derived tumor models of pancreatic cancer. ${ }^{154}$

Indatuximab ravtansine is an ADC that links an anti-SDC1 antibody to the cytotoxic agent maytansine. It was recently shown to strongly inhibit tumor growth in a high SDC1-expressing xenograph model of triple-negative breast cancer. ${ }^{184}$ The first clinical study on patients with relapsed and/or refractory multiple myeloma showed over $75 \%$ of patients achieved stable disease. ${ }^{87}$

Antibody-dependent cellular cytotoxicity (ADCC) is a mechanism of immune defense in which natural killer cells lyse target cells whose surface antigens have been bound by specific antibodies. Several therapeutic antibodies against glypican-3 have been developed for treatment of hepatocelullar carcinoma which features overexpression of glypican-3. ${ }^{230}$ The recombinant monoclonal antibody GC33 (codrituzumab) binds to the juxtamembrane domain of glypican-3 and induces ADCC. It was well tolerated and had antitumor effects in phase I trials. Although GC33 did not show benefits in a phase II trial, a phase I trial of the combination of GC33 and an immune checkpoint targeting antibody is currently in progress. ${ }^{153}$ Harada et al. ${ }^{73}$ recently developed an anti-GPC1 antibody for the treatment of esophageal squamous cell carcinoma. The antibody inhibited tumor growth via ADCC and decreased angiogenesis in patient derived tumor xenograft models.

\section{Heparanase Inhibitors}

Heparanase is upregulated in all major types of human cancer and is associated with increased tumor size, tumor angiogenesis, and metastasis. ${ }^{164}$ This has stimulated efforts to identify or design heparanase inhibitors for use in cancer therapy. Heparin derivatives and HS mimetics constitute a major class of heparanase inhibitors (recently reviewed by Lanzi and Cassinelli ${ }^{104}$ ) and several of these have progressed into clinical trials. The synthesis of small molecule heparanase inhibitors with high selectivity is also an active area of research. ${ }^{6,88}$

\section{Specific Editing of the Glycan Structure}

An emerging strategy is the design of tools that allow for precise editing of the GCX by targeting the 
glycan structure. These include small molecule inhibitors of glycan biosynthesis, modification of glycoproteins, and CRISPR-based editing of glycosyltransferases. Buffone and Weaver ${ }^{18}$ recently reviewed these new strategies for GCX editing. Munkley and Scott reviewed the targeting of glycan sialylation which is upregulated in cancer. ${ }^{144}$

\section{FUTURE DIRECTIONS}

As we have seen in this review, many important developments in our basic understanding of GCX structure, function and role in diseases have been described since the 2007 review. ${ }^{220}$ The future is wide open for continued GCX research. A few suggestions derived from this review are outlined below:

1. The implications of the Michel-Weinbaum revised Starling hypothesis for controlling fluid balance in perioperative settings where acute normovolemic hemodilution is required to compensate for blood loss or hypovolemia should be pursued as well as the role of trapped microdomains in muscle tissue.

2. The importance of HSPG GPC1 as the upstream mechanosensor in shear-induced NO production after longer exposures to shear (minutes to hours) has been reviewed, but the role of the GCX in NO production after acute (seconds to minutes) changes in shear has not been investigated.

3. The mechanism by which substrate stiffness sup presses GPC1 expression leading to endothelial dysfunction has not been thoroughly investigated.

4. While it has been shown that GPC1 is suppressed in a mouse model of aging, a hypertensive mouse or rat model has not been considered and these questions have not been addressed in human subjects.

5. STORM imaging of the GCX on ECs should be extended to examine additional GAGs and proteoglycans as well as a variety of cell types both in vitro and in vivo.

6. The role of GCX components in infectious dis eases is understudied. A question related to the recent SARS COV-2 (COVID -19) virus is whether GAGs/proteoglycans mediate binding of the virus to the host cell as has been shown for SARS COV-1. There are many other questions.

7. Does restoration of the GCX on ECs in inflam matory environments really limit or reverse atherosclerosis and related cardiovascular diseases? This should be tested extensively in animals and, if promising, in humans.

8. An important role for IF in cancer metastasis has been suggested by recent studies. Because mechanotransduction of IF by the cancer cell GCX is involved, it will be important to determine which cancers are sensitive to IF and which GCX components are the sensors and transducers of IF.

9. Degradation of the endothelial GCX has been suggested by recent studies as a requirement for cancer cell extravasation from the vasculature during metastasis. More extensive animal studies are required to demonstrate the importance of this mechanism.

10. While there has been extensive recent research to develop GCX preserving or enhancing agents for treatment of vascular and related diseases, there is a great need for additional testing in animal models of diseases as pre-clinical preparation for clinical trials.

\section{CONFLICT OF INTEREST}

Author SB, Author LMC, Author BF, and Author JMT declare that they have no conflicts of interest.

\section{REFERENCES}

${ }^{1}$ Adamson, R. H., J. F. Lenz, X. Zhang, G. N. Adamson, S. Weinbaum, and F. E. Curry. Oncotic pressures opposing filtration across non-fenestrated rat microvessels. J. Physiol. 557(Pt 3):889-907, 2004.

${ }^{2}$ Adembri, C., E. Sgambati, L. Vitali, V. Selmi, M. Margheri, A. Tani, et al. Sepsis induces albuminuria and alterations in the glomerular filtration barrier: a morphofunctional study in the rat. Crit. Care 15(6):R277, 2011.

${ }^{3}$ Aird, W. C. The role of the endothelium in severe sepsis and multiple organ dysfunction syndrome. Blood 101(10):3765-3777, 2003.

${ }^{4}$ Alves, N. G., A. N. Trujillo, J. W. Breslin, and S. Y. Yuan. Sphingosine-1-phosphate reduces hemorrhagic shock and resuscitation-induced microvascular leakage by protecting endothelial mitochondrial integrity. Shock. 52(4):423-433, 2019.

${ }^{5}$ Annecke, T., D. Chappell, C. Chen, M. Jacob, U. Welsch, C. P. Sommerhoff, et al. Sevoflurane preserves the endothelial glycocalyx against ischaemia-reperfusion injury. Br. J. Anaesth. 104(4):414-421, 2010.

${ }^{6}$ Baburajeev, C. P., C. D. Mohan, S. Rangappa, D. J. Mason, J. E. Fuchs, A. Bender, et al. Identification of novel class of triazolo-thiadiazoles as potent inhibitors of human heparanase and their anticancer activity. $B M C$ Cancer. 17(1):235, 2017.

${ }^{7}$ Baeyens, N., M. J. Mulligan-Kehoe, F. Corti, D. D. Simon, T. D. Ross, J. M. Rhodes, et al. Syndecan 4 is required for endothelial alignment in flow and atheroprotective signaling. Proc. Natl. Acad. Sci. U $S$ A. 111(48):17308-17313, 2014.

${ }^{8}$ Bailey, C. J. Metformin: effects on micro and macrovascular complications in type 2 diabetes. Cardiovasc. Drugs Ther. 22(3):215-224, 2008. 
${ }^{9}$ Bar, A., M. Targosz-Korecka, J. Suraj, B. Proniewski, A. Jasztal, B. Marczyk, et al. Degradation of glycocalyx and multiple manifestations of endothelial dysfunction coincide in the early phase of endothelial dysfunction before atherosclerotic plaque development in apolipoprotein $\mathrm{E} /$ low-density lipoprotein receptor-deficient mice. J. Am. Heart Assoc. 8(6):e011171, 2019.

${ }^{10}$ Barbareschi, M., P. Maisonneuve, D. Aldovini, M. G. Cangi, L. Pecciarini, F. Angelo Mauri, et al. High syndecan-1 expression in breast carcinoma is related to an aggressive phenotype and to poorer prognosis. Cancer 98(3):474-483, 2003.

${ }^{11}$ Barelli, S., and L. Alberio. The role of plasma transfusion in massive bleeding: protecting the endothelial glycocalyx? Front. Med. (Lausanne). 5:91, 2018.

${ }^{12}$ Bartosch, A. M. W., R. Mathews, and J. M. Tarbell. Endothelial glycocalyx-mediated nitric oxide production in response to selective AFM pulling. Biophys. $J$. 113(1):101-108, 2017.

${ }^{13}$ Basu, A., M. Du, M. J. Leyva, K. Sanchez, N. M. Betts, $\mathrm{M}$. Wu, et al. Blueberries decrease cardiovascular risk factors in obese men and women with metabolic syndrome. J. Nutr. 140(9):1582-1587, 2010.

${ }^{14}$ Bates, M., B. Huang, G. T. Dempsey, and X. Zhuang. Multicolor super-resolution imaging with photo-switchable fluorescent probes. Science 317(5845):1749-1753, 2007.

${ }^{15}$ Becker, B. F., M. Jacob, S. Leipert, A. H. Salmon, and D. Chappell. Degradation of the endothelial glycocalyx in clinical settings: searching for the sheddases. Br. J. Clin. Pharmacol. 80(3):389-402, 2015.

${ }^{16}$ Bharat, D., R. R. M. Cavalcanti, C. Petersen, N. Begaye, B. R. Cutler, M. M. A. Costa, et al. Blueberry metabolites attenuate lipotoxicity-induced endothelial dysfunction. Mol. Nutr. Food Res. 2018. https://doi.org/10.1002/mnfr. 201700601.

${ }^{17}$ Brower, J. B., J. H. Targovnik, M. R. Caplan, and S. P. Massia. High glucose-mediated loss of cell surface heparan sulfate proteoglycan impairs the endothelial shear stress response. Cytoskeleton (Hoboken). 67(3):135-141, 2010.

${ }^{18}$ Buffone, A., and V. M. Weaver. Don't sugarcoat it: how glycocalyx composition influences cancer progression. $J$. Cell Biol. 219(1):e201910070, 2020.

${ }^{19}$ Buscail, E., C. Alix-Panabieres, P. Quincy, T. Cauvin, A. Chauvet, O. Degrandi, et al. High clinical value of liquid biopsy to detect circulating tumor cells and tumor exosomes in pancreatic ductal adenocarcinoma patients eligible for up-front surgery. Cancers (Basel). 11(11):1656, 2019.

${ }^{20}$ Butler, M. J., R. Ramnath, H. Kadoya, D. Desposito, A. Riquier-Brison, J. K. Ferguson, et al. Aldosterone induces albuminuria via matrix metalloproteinase-dependent damage of the endothelial glycocalyx. Kidney Int. 95(1):94-107, 2019.

${ }^{21}$ Cai, B., J. Fan, M. Zeng, L. Zhang, and B. M. Fu. Adhesion of malignant mammary tumor cells MDA-MB231 to microvessel wall increases microvascular permeability via degradation of endothelial surface glycocalyx. J. Appl. Physiol. 113(7):1141-1153, 2012.

${ }^{22}$ Cancel, L. M., E. E. Ebong, S. Mensah, C. Hirschberg, and J. M. Tarbell. Endothelial glycocalyx, apoptosis and inflammation in an atherosclerotic mouse model. Atherosclerosis. 252:136-146, 2016.
${ }^{23}$ Cancel, L. M., and J. M. Tarbell. Rhamnan sulfate enhances the endothelial glycocalyx and decreases the LDL permeability of human coronary artery endothelial cells in vitro. FASEB J. 27:7, 2013. https://doi.org/10.1096/fa sebj.27.1_supplement.896.3.

${ }^{24}$ Chappell, D., B. Heindl, M. Jacob, T. Annecke, C. Chen, M. Rehm, et al. Sevoflurane reduces leukocyte and platelet adhesion after ischemia-reperfusion by protecting the endothelial glycocalyx. Anesthesiology 115(3):483-491, 2011.

${ }^{25}$ Chelazzi, C., G. Villa, P. Mancinelli, A. R. De Gaudio, and C. Adembri. Glycocalyx and sepsis-induced alterations in vascular permeability. Crit. Care 19(1):26, 2015.

${ }^{26}$ Chen, Y. T., Z. Chen, and Y. N. Du. Immunohistochemical analysis of RHAMM expression in normal and neoplastic human tissues: a cell cycle protein with distinctive expression in mitotic cells and testicular germ cells. Oncotarget. 9(30):20941-20952, 2018.

${ }^{27}$ Chen, L., X. Li, M. Chen, Y. Feng, and C. Xiong. The ACE2 expression in human heart indicates new potential mechanism of heart injury among patients infected with SARS-CoV-2. Cardiovasc. Res. 116(6):1097-1100, 2020.

${ }^{28}$ Chignalia, A. Z., F. Yetimakman, S. C. Christiaans, S. Unal, B. Bayrakci, B. M. Wagener, et al. The glycocalyx and trauma: a review. Shock. 45(4):338-348, 2016.

${ }^{29}$ Chistiakov, D. A., A. N. Orekhov, and Y. V. Bobryshev. Endothelial PECAM-1 and its function in vascular physiology and atherogenic pathology. Exp. Mol. Pathol. 100(3):409-415, 2016.

${ }^{30}$ Coccheri, S., and F. Mannello. Development and use of sulodexide in vascular diseases: implications for treatment. Drug Des. Devel. Ther. 8:49-65, 2013.

${ }^{31}$ Contreras, H. R., M. Fabre, F. Granes, R. CasaroliMarano, N. Rocamora, A. G. Herreros, et al. Syndecan-2 expression in colorectal cancer-derived HT-29 M6 epithelial cells induces a migratory phenotype. Biochem. Biophys. Res. Commun. 286(4):742-751, 2001.

${ }^{32}$ Conway, D. E., M. T. Breckenridge, E. Hinde, E. Gratton, C. S. Chen, and M. A. Schwartz. Fluid shear stress on endothelial cells modulates mechanical tension across VEcadherin and PECAM-1. Curr. Biol. 23(11):1024-1030, 2013.

${ }^{33}$ Coombe, D. R., S. M. Stevenson, B. F. Kinnear, N. S. Gandhi, R. L. Mancera, R. I. Osmond, et al. Platelet endothelial cell adhesion molecule 1 (PECAM-1) and its interactions with glycosaminoglycans: 2. Biochemical analyses. Biochemistry. 47(17):4863-4875, 2008.

${ }^{34}$ Cooper, S., H. Teoh, M. A. Campeau, S. Verma, and R. L. Leask. Empagliflozin restores the integrity of the endothelial glycocalyx in vitro. Mol. Cell. Biochem. 459(12):121-130, 2019.

${ }^{35}$ Couchman, J. R., H. Multhaupt, and R. D. Sanderson. Recent insights into cell surface heparan sulphate proteoglycans and cancer. F1000Res. 2016. https://doi.org/10. 12688/f1000research.8543.1.

${ }^{36}$ Cui, N., H. Wang, Y. Long, L. Su, and D. Liu. Dexamethasone suppressed LPS-induced matrix metalloproteinase and its effect on endothelial glycocalyx shedding. Mediators Inflamm. 2015:912726, 2015.

${ }^{37}$ Curry, F. E. The molecular structure of the endothelial glycocalyx layer (EGL) and surface layers (ESL) modulation of transvascular exchange. Adv. Exp. Med. Biol. 1097:29-49, 2018.

${ }^{38}$ Cutler, B. R., S. Gholami, J. S. Chua, B. Kuberan, and P. V. Anandh Babu. Blueberry metabolites restore cell sur- 
face glycosaminoglycans and attenuate endothelial inflammation in diabetic human aortic endothelial cells. Int. J. Cardiol. 261:155-158, 2018.

${ }^{39}$ Dabagh, M., P. Jalali, P. J. Butler, A. Randles, and J. M. Tarbell. Mechanotransmission in endothelial cells subjected to oscillatory and multi-directional shear flow. $J . R$. Soc. Interface. 14(130):20170185, 2017.

${ }^{40}$ Danese, C., A. R. Vestri, V. D'Alfonso, G. Deriu, S. Dispensa, R. Baldini, et al. Do hypertension and diabetes mellitus influence the site of atherosclerotic plaques? Clin. Ter. 157(1):9-13, 2006.

${ }^{41}$ Davies, P. F. Hemodynamic shear stress and the endothelium in cardiovascular pathophysiology. Nat. Clin. Pract. Cardiovasc. Med. 6(1):16-26, 2009.

${ }^{42}$ De Rossi, G., A. R. Evans, E. Kay, A. Woodfin, T. R. McKay, S. Nourshargh, et al. Shed syndecan-2 inhibits angiogenesis. J. Cell Sci. 127(Pt 21):4788-4799, 2014.

${ }^{43}$ dela Paz, N. G., B. Melchior, F. Y. Shayo, and J. A. Frangos. Heparan sulfates mediate the interaction between platelet endothelial cell adhesion molecule-1 (PECAM-1) and the $\mathrm{G} \alpha \mathrm{q} / 11$ subunits of heterotrimeric $\mathrm{G}$ proteins. J. Biol. Chem. 289(11):7413-7424, 2014.

${ }^{44}$ Dogné, S., B. Flamion, and N. Caron. Endothelial glycocalyx as a shield against diabetic vascular complications: involvement of hyaluronan and hyaluronidases. Arterioscler. Thromb. Vasc. Biol. 38(7):1427-1439, 2018.

${ }^{45}$ Dogné, S., G. Rath, F. Jouret, N. Caron, C. Dessy, and B. Flamion. Hyaluronidase 1 deficiency preserves endothelial function and glycocalyx integrity in early streptozotocininduced diabetes. Diabetes 65(9):2742-2753, 2016.

${ }^{46}$ Duan, L., X. Q. Hu, D. Y. Feng, S. Y. Lei, and G. H. Hu. GPC-1 may serve as a predictor of perineural invasion and a prognosticator of survival in pancreatic cancer. Asian J Surg. 36(1):7-12, 2013.

${ }^{47}$ Dull, R. O., M. Cluff, J. Kingston, D. Hill, H. Chen, S. Hoehne, et al. Lung heparan sulfates modulate $\mathrm{K}(\mathrm{fc})$ during increased vascular pressure: evidence for glycocalyx-mediated mechanotransduction. Am. J. Physiol. Lung Cell. Mol. Physiol. 302(9):L816-L828, 2012.

${ }^{48}$ Dull, R. O., I. Mecham, and S. McJames. Heparan sulfates mediate pressure-induced increase in lung endothelial hydraulic conductivity via nitric oxide/reactive oxygen species. Am. J. Physiol. Lung Cell. Mol. Physiol. 292(6):L1452-L1458, 2007.

${ }^{49}$ Duprez, Daniel A. Arterial stiffness and endothelial function. Hypertension 55(3):612-613, 2010.

${ }^{50}$ Ebong, E. E., S. V. Lopez-Quintero, V. Rizzo, D. C. Spray, and J. M. Tarbell. Shear-induced endothelial NOS activation and remodeling via heparan sulfate, glypican-1, and syndecan-1. Integr Biol (Camb). 6(3):338-347, 2014.

${ }^{51}$ Ebong, E. E., F. P. Macaluso, D. C. Spray, and J. M. Tarbell. Imaging the endothelial glycocalyx in vitro by rapid freezing/freeze substitution transmission electron microscopy. Arterioscler. Thromb. Vasc. Biol. 31(8):19081915, 2011.

${ }^{52}$ Erdem, M., S. Erdem, O. Sanli, H. Sak, I. Kilicaslan, F. Sahin, et al. Up-regulation of TGM2 with ITGB1 and SDC4 is important in the development and metastasis of renal cell carcinoma. Urol. Oncol. 32(1):25, 2014.

${ }^{53}$ Eskens, B. J., C. J. Zuurbier, J. van Haare, H. Vink, and J. W. van Teeffelen. Effects of two weeks of metformin treatment on whole-body glycocalyx barrier properties in db/db mice. Cardiovasc. Diabetol. 12:175, 2013.

${ }^{54}$ Estep, R. D., A. N. Govindan, M. Manoharan, H. Li, S. S. Fei, B. S. Park, et al. Molecular analysis of lymphoid tissue from rhesus macaque rhadinovirus-infected monkeys identifies alterations in host genes associated with oncogenesis. PLoS ONE 15(2):e0228484, 2020.

${ }^{55}$ Fan, J., B. Cai, M. Zeng, Y. Hao, F. G. Giancotti, and B. $\mathrm{M}$. Fu. Integrin $\beta 4$ signaling promotes mammary tumor cell adhesion to brain microvascular endothelium by inducing ErbB2-mediated secretion of VEGF. Ann. Biomed. Eng. 39(8):2223-2241, 2011.

${ }^{56}$ Fan, J., and B. M. Fu. Quantification of malignant breast cancer cell MDA-MB-231 transmigration across brain and lung microvascular endothelium. Ann. Biomed. Eng. 44(7):2189-2201, 2016.

${ }^{57}$ Fan, J., Y. Sun, Y. Xia, J. M. Tarbell, and B. M. Fu. Endothelial surface glycocalyx (ESG) components and ultra-structure revealed by stochastic optical reconstruction microscopy (STORM). Biorheology 56(2-3):77-88, 2019.

${ }^{58}$ Fink, M. P. Animal models of sepsis. Virulence. 5(1):143$153,2014$.

${ }^{59}$ Fleming, I., B. Fisslthaler, M. Dixit, and R. Busse. Role of PECAM-1 in the shear-stress-induced activation of Akt and the endothelial nitric oxide synthase (eNOS) in endothelial cells. J. Cell Sci. 118(Pt 18):4103-4111, 2005.

${ }^{60}$ Florian, J. A., J. R. Kosky, K. Ainslie, Z. Pang, R. O. Dull, and J. M. Tarbell. Heparan sulfate proteoglycan is a mechanosensor on endothelial cells. Circ. Res. 93(10):e136-e142, 2003.

${ }^{61} \mathrm{Fu}$, B. M., and J. M. Tarbell. Mechano-sensing and transduction by endothelial surface glycocalyx: composition, structure, and function. Wiley Interdiscip. Rev. Syst. Biol. Med. 5(3):381-390, 2013.

${ }^{62}$ Gallagher, J. Fell-Muir lecture: heparan sulphate and the art of cell regulation: a polymer chain conducts the protein orchestra. Int. J. Exp. Pathol. 96(4):203-231, 2015.

${ }^{63}$ Goligorsky, M. S., and D. Sun. Glycocalyx in endotoxemia and sepsis. Am. J. Pathol. 190(4):791-798, 2020.

${ }^{64}$ Guerci, P., B. Ergin, Z. Uz, Y. Ince, M. Westphal, M. Heger, et al. Glycocalyx degradation is independent of vascular barrier permeability increase in nontraumatic hemorrhagic shock in rats. Anesth. Analg. 129(2):598-607, 2019.

${ }^{65}$ Guo, W., and F. G. Giancotti. Integrin signalling during tumour progression. Nat. Rev. Mol. Cell Biol. 5(10):816826, 2004.

${ }^{66}$ Guo, J., Z. C. Yang, and Y. Liu. Attenuating pulmonary hypertension by protecting the integrity of glycocalyx in rats model of pulmonary artery hypertension. Inflammation. 42(6):1951-1956, 2019.

${ }^{67}$ Guo, M., H. Zhang, J. Zheng, and Y. Liu. Glypican-3: a new target for diagnosis and treatment of hepatocellular carcinoma. J. Cancer. 11(8):2008-2021, 2020.

${ }^{68}$ Guyton, H. G., and H. M. Decker. Respiratory protection provided by five new contagion masks. Appl. Microbiol. 11(1):66-68, 1963.

${ }^{69}$ Haeren, R. H., S. E. van de Ven, M. A. van Zandvoort, H. Vink, J. J. van Overbeeke, G. Hoogland, et al. Assessment and imaging of the cerebrovascular glycocalyx. Curr. Neurovasc. Res. 13(3):249-260, 2016.

${ }^{70}$ Haessler, U., J. C. Teo, D. Foretay, P. Renaud, and M. A. Swartz. Migration dynamics of breast cancer cells in a tunable 3D interstitial flow chamber. Integr. Biol. (Camb). 4(4):401-409, 2012.

${ }^{71}$ Hammond, E., A. Khurana, V. Shridhar, and K. Dredge. The role of heparanase and sulfatases in the modification of heparan sulfate proteoglycans within the tumor 
microenvironment and opportunities for novel cancer therapeutics. Front. Oncol. 4:195, 2014.

${ }^{72}$ Hara, H., T. Takahashi, S. Serada, M. Fujimoto, T. Ohkawara, R. Nakatsuka, et al. Overexpression of glypican-1 implicates poor prognosis and their chemoresistance in oesophageal squamous cell carcinoma. $\mathrm{Br}$. J. Cancer 115(1):66-75, 2016.

${ }^{73}$ Harada, E., S. Serada, M. Fujimoto, Y. Takahashi, T. Takahashi, H. Hara, et al. Glypican-1 targeted antibodybased therapy induces preclinical antitumor activity against esophageal squamous cell carcinoma. Oncotarget. 8(15):24741-24752, 2017.

${ }^{74}$ Harding, I. C., R. Mitra, S. A. Mensah, I. M. Herman, and E. E. Ebong. Pro-atherosclerotic disturbed flow disrupts caveolin-1 expression, localization, and function via glycocalyx degradation. J. Transl. Med. 16(1):364, 2018.

${ }^{75}$ Hempel, C., J. Sporring, and J. A. L. Kurtzhals. Experimental cerebral malaria is associated with profound loss of both glycan and protein components of the endothelial glycocalyx. FASEB J. 33(2):2058-2071, 2019.

${ }^{76}$ Hippensteel, J. A., B. J. Anderson, J. E. Orfila, S. A. McMurtry, R. M. Dietz, G. Su, et al. Circulating heparan sulfate fragments mediate septic cognitive dysfunction. $J$ Clin Invest. 129(4):1779-1784, 2019.

${ }^{77} \mathrm{Hu}$, X., R. H. Adamson, B. Liu, F. E. Curry, and S. Weinbaum. Starling forces that oppose filtration after tissue oncotic pressure is increased. Am. J. Physiol. Heart Circ. Physiol. 279(4):H1724-H1736, 2000.

${ }^{78} \mathrm{Hu}, \mathrm{X}$., and S. Weinbaum. A new view of Starling's hypothesis at the microstructural level. Microvasc. Res. 58(3):281-304, 1999.

${ }^{79}$ Hua, C. T., J. R. Gamble, M. A. Vadas, and D. E. Jackson. Recruitment and activation of SHP-1 proteintyrosine phosphatase by human platelet endothelial cell adhesion molecule-1 (PECAM-1). Identification of immunoreceptor tyrosine-based inhibitory motif-like binding motifs and substrates. J. Biol. Chem. 273(43):28332-28340, 1998.

${ }^{80}$ Huang, Y., K. M. Jan, D. Rumschitzki, and S. Weinbaum. Structural changes in rat aortic intima due to transmural pressure. J. Biomech. Eng. 120(4):476-483, 1998.

${ }^{81}$ Huang, Y., D. Rumschitzki, S. Chien, and S. Weinbaum. A fiber matrix model for the filtration through fenestral pores in a compressible arterial intima. Am. J. Physiol. 272(4 Pt 2):H2023-H2039, 1997.

${ }^{82}$ Huang, Y. L., C. K. Tung, A. Zheng, B. J. Kim, and M. $\mathrm{Wu}$. Interstitial flows promote amoeboid over mesenchymal motility of breast cancer cells revealed by a three dimensional microfluidic model. Integr. Biol. (Camb). 7(11):1402-1411, 2015

${ }^{83}$ Ikonomidis, I., G. Pavlidis, J. Thymis, D. Birba, A. Kalogeris, F. Kousathana, et al. Effects of glucagon-like peptide-1 receptor agonists, sodium-glucose cotransporter-2 inhibitors, and their combination on endothelial glycocalyx, arterial function, and myocardial work index in patients with type 2 diabetes mellitus after 12-month treatment. J. Am. Heart Assoc. 9(9):e015716, 2020.

${ }^{84}$ Ikonomidis, I., A. Voumvourakis, G. Makavos, H. Triantafyllidi, G. Pavlidis, K. Katogiannis, et al. Association of impaired endothelial glycocalyx with arterial stiffness, coronary microcirculatory dysfunction, and abnormal myocardial deformation in untreated hypertensives. $J$. Clin. Hypertens. (Greenwich). 20(4):672-679, 2018.
${ }^{85}$ Introini, V., A. Carciati, G. Tomaiuolo, P. Cicuta, and S. Guido. Endothelial glycocalyx regulates cytoadherence in Plasmodium falciparum malaria. J. R. Soc. Interface 15(149):20180773, 2018.

${ }^{86}$ Iozzo, R. V., and R. D. Sanderson. Proteoglycans in cancer biology, tumour microenvironment and angiogenesis. J. Cell Mol. Med. 15(5):1013-1031, 2011.

${ }^{87}$ Jagannath, S., L. T. Heffner, Jr, S. Ailawadhi, N. C. Munshi, T. M. Zimmerman, J. Rosenblatt, et al. Indatuximab ravtansine (BT062) monotherapy in patients with relapsed and/or refractory multiple myeloma. Clin. Lymphoma Myeloma Leuk. 19(6):372-380, 2019.

${ }^{88} \mathrm{Jia}$, L., and S. Ma. Recent advances in the discovery of heparanase inhibitors as anti-cancer agents. Eur. J. Med. Chem. 121:209-220, 2016.

${ }^{89}$ Jian, Y. S., C. W. Chen, C. A. Lin, H. P. Yu, H. Y. Lin, M. Y. Liao, et al. Hyaluronic acid-nimesulide conjugates as anticancer drugs against CD44-overexpressing HT-29 colorectal cancer in vitro and in vivo. Int. J. Nanomed. 12:2315-2333, 2017.

${ }^{90}$ Johansson, P. I., J. Stensballe, L. S. Rasmussen, and S. R. Ostrowski. A high admission syndecan-1 level, a marker of endothelial glycocalyx degradation, is associated with inflammation, protein $\mathrm{C}$ depletion, fibrinolysis, and increased mortality in trauma patients. Ann. Surg. 254(2):194-200, 2011.

${ }^{91}$ Jung, O., V. Trapp-Stamborski, A. Purushothaman, H. Jin, H. Wang, R. D. Sanderson, et al. Heparanase-induced shedding of syndecan-1/CD138 in myeloma and endothelial cells activates VEGFR2 and an invasive phenotype: prevention by novel synstatins. Oncogenesis. 5:e202, 2016.

${ }^{92}$ Karousou, E., S. Misra, S. Ghatak, K. Dobra, M. Gotte, D. Vigetti, et al. Roles and targeting of the HAS/ hyaluronan/CD44 molecular system in cancer. Matrix Biol. 59:3-22, 2017.

${ }^{93}$ Kazuma, S., Y. Tokinaga, M. Kimizuka, R. Azumaguchi, K. Hamada, and M. Yamakage. Sevoflurane promotes regeneration of the endothelial glycocalyx by upregulating sialyltransferase. J. Surg. Res. 241:40-47, 2019.

${ }^{94}$ Kim, Y. H., P. Nijst, K. Kiefer, and W. H. Tang. Endothelial glycocalyx as biomarker for cardiovascular diseases: mechanistic and clinical implications. Curr. Heart Fail. Rep. 14(2):117-126, 2017.

${ }^{95}$ Kind, S., C. Merenkow, F. Buscheck, K. Moller, D. Dum, V. Chirico, et al. Prevalence of Syndecan-1 (CD138) expression in different kinds of human tumors and normal tissues. Dis. Markers 2019:4928315, 2019.

${ }^{96}$ Kingsmore, K. M., D. K. Logsdon, D. H. Floyd, S. M. Peirce, B. W. Purow, and J. M. Munson. Interstitial flow differentially increases patient-derived glioblastoma stem cell invasion via CXCR4, CXCL12, and CD44-mediated mechanisms. Integr. Biol. (Camb). 8(12):1246-1260, 2016.

${ }^{97}$ Kleeff, J., T. Ishiwata, A. Kumbasar, H. Friess, M. W. Buchler, A. D. Lander, et al. The cell-surface heparan sulfate proteoglycan glypican-1 regulates growth factor action in pancreatic carcinoma cells and is overexpressed in human pancreatic cancer. J. Clin. Invest. 102(9):16621673, 1998.

${ }^{98}$ Kuchan, M. J., and J. A. Frangos. Role of calcium and calmodulin in flow-induced nitric oxide production in endothelial cells. Am. J. Physiol. 266(3 Pt 1):C628-C636, 1994.

${ }^{99}$ Kumase, F., Y. Morizane, S. Mohri, I. Takasu, A. Ohtsuka, and H. Ohtsuki. Glycocalyx degradation in retinal 
and choroidal capillary endothelium in rats with diabetes and hypertension. Acta Med. Okayama 64(5):277-283, 2010.

${ }^{100}$ Kusche-Vihrog, K., and H. Oberleithner. An emerging concept of vascular salt sensitivity. Biol. Rep. 4:20, 2012.

${ }^{101}$ Landis, H. R. Laboratory and clinical methods. Trans. Am. Climatol. Clin. Assoc. 43:134-143, 1927.

${ }^{102}$ Landman, G. W., N. Kleefstra, K. J. van Hateren, K. H. Groenier, R. O. Gans, and H. J. Bilo. Metformin associated with lower cancer mortality in type 2 diabetes: ZODIAC-16. Diabetes Care 33(2):322-326, 2010.

${ }^{103}$ Lang, J., N. Yang, J. Deng, K. Liu, P. Yang, G. Zhang, et al. Inhibition of SARS pseudovirus cell entry by lactoferrin binding to heparan sulfate proteoglycans. PLoS ONE 6(8):e23710, 2011.

${ }^{104}$ Lanzi, C., and G. Cassinelli. Heparan sulfate mimetics in cancer therapy: the challenge to define structural determinants and the relevance of targets for optimal activity. Molecules. 23(11):2915, 2018.

${ }^{105}$ Lauster, D., M. Glanz, M. Bardua, K. Ludwig, M. Hellmund, U. Hoffmann, et al. Multivalent peptide-nanoparticle conjugates for influenza-virus inhibition. Angew. Chem. Int. Ed. Engl. 56(21):5931-5936, 2017.

${ }^{106}$ Leskova, W., H. Pickett, R. S. Eshaq, B. Shrestha, C. B. Pattillo, and N. R. Harris. Effect of diabetes and hyaluronidase on the retinal endothelial glycocalyx in mice. Exp. Eye Res. 179:125-131, 2019.

${ }^{107}$ Levick, J. R. Capillary filtration-absorption balance reconsidered in light of dynamic extravascular factors. Exp. Physiol. 76(6):825-857, 1991.

${ }^{108}$ Levick, J. R., and C. C. Michel. Microvascular fluid exchange and the revised Starling principle. Cardiovasc. Res. 87(2):198-210, 2010.

${ }^{109}$ Lewis, J. C., R. G. Taylor, N. D. Jones, R. W. St Clair, and J. F. Cornhill. Endothelial surface characteristics in pigeon coronary artery atherosclerosis. I. Cellular alterations during the initial stages of dietary cholesterol challenge. Lab Invest. 46(2):123-138, 1982.

${ }^{110}$ Li, J., Y. Chen, X. Guo, L. Zhou, Z. Jia, Z. Peng, et al. GPC1 exosome and its regulatory miRNAs are specific markers for the detection and target therapy of colorectal cancer. J. Cell Mol. Med. 21(5):838-847, 2017.

${ }^{111} \mathrm{Li}$, J., B. Li, C. Ren, Y. Chen, X. Guo, L. Zhou, et al. The clinical significance of circulating GPC1 positive exosomes and its regulative miRNAs in colon cancer patients. Oncotarget. 8(60):101189-101202, 2017.

${ }^{112} \mathrm{Li}, \mathrm{T}$., X. Liu, Z. Zhao, L. Ni, and C. Liu. Sulodexide recovers endothelial function through reconstructing glycocalyx in the balloon-injury rat carotid artery model. Oncotarget. 8(53):91350-91361, 2017.

${ }^{113}$ Li, J., T. Yuan, X. Zhao, G. Y. Lv, and H. Q. Liu. Protective effects of sevoflurane in hepatic ischemia-reperfusion injury. Int. J. Immunopathol. Pharmacol. 29(2):300307,2016

${ }^{114}$ Ligi, D., L. Croce, and F. Mannello. Chronic venous disorders: the dangerous, the good, and the diverse. Int. J. Mol. Sci. 19(9):2544, 2018.

${ }^{115} \mathrm{Lim}$, H. C., and J. R. Couchman. Syndecan-2 regulation of morphology in breast carcinoma cells is dependent on RhoGTPases. Biochim. Biophys. Acta 1840(8):2482-2490, 2014.

${ }^{116}$ Lopez-Quintero, S. V., L. M. Cancel, A. Pierides, D. Antonetti, D. C. Spray, and J. M. Tarbell. High glucose attenuates shear-induced changes in endothelial hydraulic conductivity by degrading the glycocalyx. PLoS ONE 8(11):e78954, 2013.

${ }^{117}$ Lu, H., F. Niu, F. Liu, J. Gao, Y. Sun, and X. Zhao. Elevated glypican-1 expression is associated with an unfavorable prognosis in pancreatic ductal adenocarcinoma. Cancer Med. 6(6):1181-1191, 2017.

${ }^{118}$ Lygizos, M. I., Y. Yang, C. J. Altmann, K. Okamura, A. A. Hernando, M. J. Perez, et al. Heparanase mediates renal dysfunction during early sepsis in mice. Physiol. Rep. 1(6):e00153, 2013.

${ }^{119}$ Machin, D. R., S. I. Bloom, R. A. Campbell, T. T. T. Phuong, P. E. Gates, L. A. Lesniewski, et al. Advanced age results in a diminished endothelial glycocalyx. Am. J. Physiol. Heart Circ. Physiol. 315(3):H531-H539, 2018.

${ }^{120}$ Mahmoud, M., M. Mayer, L. M. Cancel, A. M. Bartosch, R. Mathews, and J. M. Tarbell. The Glycocalyx core protein Glypican 1 protects vessel wall endothelial cells from stiffness-mediated dysfunction and disease. Cardiovasc. Res. 20:10, 2020. https://doi.org/10.1093/cvr/cvaa20 1.

${ }^{121}$ Majerczak, J., K. Duda, S. Chlopicki, G. Bartosz, A. Zakrzewska, A. Balcerczyk, et al. Endothelial glycocalyx integrity is preserved in young, healthy men during a single bout of strenuous physical exercise. Physiol. Res. 65(2):281-291, 2016.

${ }^{122}$ Majerczak, J., M. Grandys, K. Duda, A. Zakrzewska, A. Balcerczyk, L. Kolodziejski, et al. Moderate-intensity endurance training improves endothelial glycocalyx layer integrity in healthy young men. Exp. Physiol. 102(1):7085, 2017.

${ }^{123}$ Mansilha, A., and J. Sousa. Pathophysiological mechanisms of chronic venous disease and implications for venoactive drug therapy. Int. J. Mol. Sci. 19(6):1669, 2018.

${ }^{124}$ Maruhashi, T., J. Soga, N. Fujimura, N. Idei, S. Mikami, Y. Iwamoto, et al. Endothelial dysfunction, increased arterial stiffness, and cardiovascular risk prediction in patients with coronary artery disease: FMD-J (Flow-Mediated Dilation Japan) Study A. J. Am. Heart Assoc. 7(14):e008588, 2018.

${ }^{125}$ Masuda, M., M. Osawa, H. Shigematsu, N. Harada, and K. Fujiwara. Platelet endothelial cell adhesion molecule-1 is a major SH-PTP2 binding protein in vascular endothelial cells. FEBS Lett. 408(3):331-336, 1997.

${ }^{126}$ Matsuda, K., H. Maruyama, F. Guo, J. Kleeff, J. Itakura, Y. Matsumoto, et al. Glypican-1 is overexpressed in human breast cancer and modulates the mitogenic effects of multiple heparin-binding growth factors in breast cancer cells. Cancer Res. 61(14):5562-5569, 2001.

${ }^{127}$ Matsuzaki, S., S. Serada, K. Hiramatsu, S. Nojima, Y. Ueda, T. Ohkawara, et al. Anti-glypican-1 antibody-drug conjugate exhibits potent preclinical antitumor activity against glypican-1 positive uterine cervical cancer. Int. J. Cancer 142(5):1056-1066, 2018.

${ }^{128}$ Matthay, M. A., R. L. Zemans, G. A. Zimmerman, Y. M. Arabi, J. R. Beitler, A. Mercat, et al. Acute respiratory distress syndrome. Nat. Rev. Dis. Primers. 5(1):18, 2019.

${ }^{129}$ McGarrity, S., Ó. Anuforo, H. Halldórsson, A. Bergmann, S. Halldórsson, S. Palsson, et al. Metabolic systems analysis of LPS induced endothelial dysfunction applied to sepsis patient stratification. Sci. Rep. 8(1):6811, 2018.

${ }^{130}$ Mele, V., L. Sokol, V. H. Kolzer, D. Pfaff, M. G. Muraro, I. Keller, et al. The hyaluronan-mediated motility receptor RHAMM promotes growth, invasiveness and dissemination of colorectal cancer. Oncotarget. 8(41):70617-70629, 2017. 
${ }^{131}$ Melo, S. A., L. B. Luecke, C. Kahlert, A. F. Fernandez, S. T. Gammon, J. Kaye, et al. Glypican-1 identifies cancer exosomes and detects early pancreatic cancer. Nature 523(7559): 177-182, 2015.

${ }^{132}$ Mensah, S. A., M. J. Cheng, H. Homayoni, B. D. Plouffe, A. J. Coury, and E. E. Ebong. Regeneration of glycocalyx by heparan sulfate and sphingosine 1-phosphate restores inter-endothelial communication. PLOS ONE 12(10):e0186116, 2017.

${ }^{133}$ Michel, C. C. Fluid movement through capillary walls. Handbook of Physiology: Sect 2. Philadelphia: American Physiological Society, 1984.

${ }^{134}$ Michel, C. C. Starling: the formulation of his hypothesis of microvascular fluid exchange and its significance after 100 years. Exp. Physiol. 82(1):1-30, 1997.

${ }^{135}$ Michel, C. C., and M. E. Phillips. Steady-state fluid filtration at different capillary pressures in perfused frog mesenteric capillaries. J. Physiol. 388:421-435, 1987.

${ }^{136}$ Milewska, A., M. Zarebski, P. Nowak, K. Stozek, J. Potempa, and K. Pyrc. Human coronavirus NL63 utilizes heparan sulfate proteoglycans for attachment to target cells. J. Virol. 88(22):13221-13230, 2014

${ }^{137}$ Mishra, H. K., J. Ma, and B. Walcheck. Ectodomain Shedding by ADAM17: its role in neutrophil recruitment and the impairment of this process during sepsis. Front Cell Infect. Microbiol. 7:138, 2017.

${ }^{138}$ Misra, S., V. C. Hascall, R. R. Markwald, and S. Ghatak. Interactions between Hyaluronan and Its Receptors (CD44, RHAMM) Regulate the Activities of Inflammation and Cancer. Front Immunol. 6:201, 2015.

${ }^{139}$ Misra, S., P. Heldin, V. C. Hascall, N. K. Karamanos, S. S. Skandalis, R. R. Markwald, et al. Hyaluronan-CD44 interactions as potential targets for cancer therapy. FEBS J. 278(9):1429-1443, 2011.

${ }^{140}$ Mitchell, G. F. Arterial stiffness and hypertension: chicken or egg? Hypertension 64(2):210-214, 2014.

${ }^{141}$ Mitchell, G. F., S.-J. Hwang, R. S. Vasan, M. G. Larson, M. J. Pencina, N. M. Hamburg, et al. Arterial stiffness and cardiovascular events: the Framingham Heart Study. Circulation 121(4):505-511, 2010.

${ }^{142}$ Moran, H., L. M. Cancel, M. A. Mayer, H. Qazi, L. L. Munn, and J. M. Tarbell. The cancer cell glycocalyx proteoglycan Glypican-1 mediates interstitial flow mechanotransduction to enhance cell migration and metastasis. Biorheology 56(2-3):151-161, 2019.

${ }^{143}$ Munkley, J., and D. J. Elliott. Hallmarks of glycosylation in cancer. Oncotarget. 7(23):35478-35489, 2016.

${ }^{144}$ Munkley, J., and E. Scott. Targeting aberrant sialylation to treat cancer. Medicines (Basel). 6(4):102, 2019.

${ }^{145}$ Munson, J. M., R. V. Bellamkonda, and M. A. Swartz. Interstitial flow in a 3D microenvironment increases glioma invasion by a CXCR4-dependent mechanism. Cancer Res. 73(5):1536-1546, 2013.

${ }^{146}$ Munson, J. M., and A. C. Shieh. Interstitial fluid flow in cancer: implications for disease progression and treatment. Cancer Manag. Res. 6:317-328, 2014.

${ }^{147}$ Nägga, K., O. Hansson, D. van Westen, L. Minthon, and M. Wennström. Increased levels of hyaluronic acid in cerebrospinal fluid in patients with vascular dementia. $J$. Alzheimers Dis. 42(4):1435-1441, 2014.

${ }^{148}$ Nagy, N., T. Freudenberger, A. Melchior-Becker, K. Röck, M. Ter Braak, H. Jastrow, et al. Inhibition of hyaluronan synthesis accelerates murine atherosclerosis: novel insights into the role of hyaluronan synthesis. Circulation 122(22):2313-2322, 2010.
${ }^{149}$ Nelson, A., I. Berkestedt, and M. Bodelsson. Circulating glycosaminoglycan species in septic shock. Acta Anaesthesiol. Scand. 58(1):36-43, 2014.

${ }^{150}$ Neves, F. M., G. C. Meneses, N. E. Sousa, R. R. Menezes, M. C. Parahyba, A. M. Martins, et al. Syndecan-1 in acute decompensated heart failure-association with renal function and mortality. Circ. J. 79(7):1511-1519, 2015.

${ }^{151}$ Nieuwdorp, M., M. C. Meuwese, H. L. Mooij, M. H. van Lieshout, A. Hayden, M. Levi, et al. Tumor necrosis factor-alpha inhibition protects against endotoxin-induced endothelial glycocalyx perturbation. Atherosclerosis. 202(1):296-303, 2009.

${ }^{152}$ Nijst, P., J. Cops, P. Martens, Q. Swennen, M. Dupont, W. H. W. Tang, et al. Endovascular shedding markers in patients with heart failure with reduced ejection fraction: results from a single-center exploratory study. Microcirculation. 25:2, 2018. https://doi.org/10.3390/jcm7110400.

${ }^{153}$ Nishida, T., and H. Kataoka. Glypican 3-targeted therapy in hepatocellular carcinoma. Cancers (Basel). 11(9):1339, 2019.

${ }^{154}$ Nishigaki, T., T. Takahashi, S. Serada, M. Fujimoto, T. Ohkawara, H. Hara, et al. Anti-glypican-1 antibody-drug conjugate is a potential therapy against pancreatic cancer. Br. J. Cancer 122(9):1333-1341, 2020.

${ }^{155}$ Oberleithner, H. Vascular endothelium: a vulnerable transit zone for merciless sodium. Nephrol. Dial. Transplant. 29(2):240-246, 2014.

${ }^{156}$ Okamoto, T., N. Akita, M. Terasawa, T. Hayashi, and K. Suzuki. Rhamnan sulfate extracted from Monostroma nitidum attenuates blood coagulation and inflammation of vascular endothelial cells. J. Nat. Med. 73(3):614-619, 2019.

${ }^{157}$ Osawa, M., M. Masuda, N. Harada, R. B. Lopes, and K. Fujiwara. Tyrosine phosphorylation of platelet endothelial cell adhesion molecule-1 (PECAM-1, CD31) in mechanically stimulated vascular endothelial cells. Eur. J. Cell Biol. 72(3):229-237, 1997.

${ }^{158}$ Padberg, J. S., A. Wiesinger, G. S. di Marco, S. Reuter, A. Grabner, D. Kentrup, et al. Damage of the endothelial glycocalyx in chronic kidney disease. Atherosclerosis. 234(2):335-343, 2014.

${ }^{159}$ Pahakis, M. Y., J. R. Kosky, R. O. Dull, and J. M. Tarbell. The role of endothelial glycocalyx components in mechanotransduction of fluid shear stress. Biochem. Biophys. Res. Commun. 355(1):228-233, 2007.

${ }^{160}$ Pang, Z., and J. M. Tarbell. In vitro study of Starling's hypothesis in a cultured monolayer of bovine aortic endothelial cells. J. Vasc. Res. 40(4):351-358, 2003.

${ }^{161}$ Pappenheimer, J. R., and A. Soto-Rivera. Effective osmotic pressure of the plasma proteins and other quantities associated with the capillary circulation in the hindlimbs of cats and dogs. Am. J. Physiol. 152(3):471-491, 1948.

${ }^{162}$ Park, H., Y. Kim, Y. Lim, I. Han, and E. S. Oh. Syndecan-2 mediates adhesion and proliferation of colon carcinoma cells. J. Biol. Chem. 277(33):29730-29736, 2002.

${ }^{163}$ Paszek, M. J., C. C. DuFort, O. Rossier, R. Bainer, J. K. Mouw, K. Godula, et al. The cancer glycocalyx mechanically primes integrin-mediated growth and survival. $\mathrm{Na}$ ture 511(7509):319-325, 2014.

${ }^{164}$ Pisano, C., I. Vlodavsky, N. Ilan, and F. Zunino. The potential of heparanase as a therapeutic target in cancer. Biochem. Pharmacol. 89(1):12-19, 2014.

${ }^{165}$ Piva, S., V. A. McCreadie, and N. Latronico. Neuroinflammation in sepsis: sepsis associated delirium. Cardiovasc. Hematol. Disord.: Drug Targets 15(1):10-18, 2015. 
${ }^{166}$ Polikarpov, D., L. Liang, A. Care, A. Sunna, D. Campbell, B. Walsh, et al. Functionalized upconversion nanoparticles for targeted labelling of bladder cancer cells. Biomolecules. 9(12):820, 2019.

${ }^{167}$ Puerta-Guardo, H., D. R. Glasner, D. A. Espinosa, S. B. Biering, M. Patana, K. Ratnasiri, et al. Flavivirus NS1 triggers tissue-specific vascular endothelial dysfunction reflecting disease tropism. Cell Rep. 26(6):1598-1613, 2019.

${ }^{168}$ Puerta-Guardo, H., D. R. Glasner, and E. Harris. Dengue virus NS1 disrupts the endothelial glycocalyx, leading to hyperpermeability. PLoS Pathog. 12(7):e1005738, 2016.

${ }^{169}$ Qazi, H., R. Palomino, Z. D. Shi, L. L. Munn, and J. M. Tarbell. Cancer cell glycocalyx mediates mechanotransduction and flow-regulated invasion. Integr. Biol. (Camb). 5(11):1334-1343, 2013.

${ }^{170}$ Qazi, H., Z. D. Shi, J. W. Song, L. M. Cancel, P. Huang, Y. Zeng, et al. Heparan sulfate proteoglycans mediate renal carcinoma metastasis. Int. J. Cancer 139(12):27912801, 2016.

${ }^{171}$ Qazi, H., Z. D. Shi, and J. M. Tarbell. Fluid shear stress regulates the invasive potential of glioma cells via modulation of migratory activity and matrix metalloproteinase expression. PLoS ONE 6(5):e20348, 2011.

${ }^{172}$ Rai, S., Z. Nejadhamzeeigilani, N. J. Gutowski, and J. L. Whatmore. Loss of the endothelial glycocalyx is associated with increased E-selectin mediated adhesion of lung tumour cells to the brain microvascular endothelium. $J$. Exp. Clin. Cancer Res. 34:105, 2015.

${ }^{173}$ Ramnath, R., R. R. Foster, Y. Qiu, G. Cope, M. J. Butler, A. H. Salmon, et al. Matrix metalloproteinase 9-mediated shedding of syndecan 4 in response to tumor necrosis factor $\alpha$ : a contributor to endothelial cell glycocalyx dysfunction. FASEB J. 28(11):4686-4699, 2014.

${ }^{174}$ Reyes-Soffer, G., S. Holleran, M. R. Di Tullio, S. Homma, B. Boden-Albala, R. Ramakrishnan, et al. Endothelial function in individuals with coronary artery disease with and without type 2 diabetes mellitus. Metabolism. 59(9):1365-1371, 2010.

${ }^{175}$ Richter, V., M. D. Savery, M. Gassmann, O. Baum, E. R. Damiano, and A. R. Pries. Excessive erythrocytosis compromises the blood-endothelium interface in erythropoietin-overexpressing mice. J. Physiol. 589(Pt 21):51815192, 2011.

${ }^{176}$ Russell-Puleri, S., N. G. Dela Paz, D. Adams, M. Chattopadhyay, L. Cancel, E. Ebong, et al. Fluid shear stress induces upregulation of $\mathrm{COX}-2$ and $\mathrm{PGI}(2)$ release in endothelial cells via a pathway involving PECAM-1, PI3K, FAK, and p38. Am. J. Physiol. Heart Circ. Physiol. 312(3):H485-H500, 2017.

${ }^{177}$ Rzhevskiy, A. S., S. Razavi Bazaz, L. Ding, A. Kapitannikova, N. Sayyadi, D. Campbell, et al. Rapid and labelfree isolation of tumour cells from the urine of patients with localised prostate cancer using inertial microfluidics. Cancers (Basel). 12(1):81, 2019.

${ }^{178}$ Saito, T., K. Sugiyama, S. Hama, F. Yamasaki, T. Takayasu, R. Nosaka, et al. High expression of glypican-1 predicts dissemination and poor prognosis in glioblastomas. World Neurosurg. 105:282-288, 2017.

${ }^{179}$ Salmon, A. H., J. K. Ferguson, J. L. Burford, H. Gevorgyan, D. Nakano, S. J. Harper, et al. Loss of the endothelial glycocalyx links albuminuria and vascular dysfunction. J. Am. Soc. Nephrol. 23(8):1339-1350, 2012.

${ }^{180}$ Sardu, C., J. Gambardella, M. B. Morelli, X. Wang, R. Marfella, and G. Santulli. Hypertension, thrombosis, kidney failure, and diabetes: is COVID-19 an endothelial disease? A comprehensive evaluation of clinical and basic evidence. J. Clin. Med. 9(5):1417, 2020.

${ }^{181}$ Schierke, F., M. J. Wyrwoll, M. Wisdorf, L. Niedzielski, M. Maase, T. Ruck, et al. Nanomechanics of the endothelial glycocalyx contribute to $\mathrm{Na}(+)$-induced vascular inflammation. Sci. Rep. 7:46476, 2017.

${ }^{182}$ Schmidt, E. P., Y. Yang, W. J. Janssen, A. Gandjeva, M. J. Perez, L. Barthel, et al. The pulmonary endothelial glycocalyx regulates neutrophil adhesion and lung injury during experimental sepsis. Nat. Med. 18(8):1217-1223, 2012.

${ }^{183}$ Schmitz, B., H. Niehues, M. Lenders, L. Thorwesten, A. Klose, M. Krüger, et al. Effects of high-intensity interval training on microvascular glycocalyx and associated microRNAs. Am. J. Physiol. Heart Circ. Physiol. 316(6):H1538-H1551, 2019.

${ }^{184}$ Schonfeld, K., P. Herbener, C. Zuber, T. Hader, K. Bernoster, C. Uherek, et al. Activity of indatuximab ravtansine against triple-negative breast cancer in preclinical tumor models. Pharm. Res. 35(6):118, 2018.

${ }^{185}$ Secomb, T. W., R. Hsu, and A. R. Pries. Effect of the endothelial surface layer on transmission of fluid shear stress to endothelial cells. Biorheology 38(2-3):143-150, 2001.

${ }^{186}$ Sehba, F. A., W. H. Ding, I. Chereshnev, and J. B. Bederson. Effects of S-nitrosoglutathione on acute vasoconstriction and glutamate release after subarachnoid hemorrhage. Stroke 30(9):1955-1961, 1999.

${ }^{187}$ Shen, S., J. Fan, B. Cai, Y. Lv, M. Zeng, Y. Hao, et al. Vascular endothelial growth factor enhances cancer cell adhesion to microvascular endothelium in vivo. Exp. Physiol. 95(2):369-379, 2010.

${ }^{188}$ Shields, J. D., M. E. Fleury, C. Yong, A. A. Tomei, G. J. Randolph, and M. A. Swartz. Autologous chemotaxis as a mechanism of tumor cell homing to lymphatics via interstitial flow and autocrine CCR7 signaling. Cancer Cell 11(6):526-538, 2007.

${ }^{189}$ Shinyo, Y., J. Kodama, T. Hasengaowa, T. Kusumoto, and Y. Hiramatsu. Loss of cell-surface heparan sulfate expression in both cervical intraepithelial neoplasm and invasive cervical cancer. Gynecol. Oncol. 96(3):776-783, 2005.

${ }^{190}$ Simizu, S., K. Ishida, and H. Osada. Heparanase as a molecular target of cancer chemotherapy. Cancer Sci. 95(7):553-558, 2004.

${ }^{191}$ Soares, M. A., F. C. Teixeira, M. Fontes, A. L. Areas, M. G. Leal, M. S. Pavao, et al. Heparan sulfate proteoglycans may promote or inhibit cancer progression by interacting with integrins and affecting cell migration. Biomed. Res. Int. 2015:453801, 2015

${ }^{192}$ Son, D. J., S. Kumar, W. Takabe, C. W. Kim, C. W. Ni, N. Alberts-Grill, et al. The atypical mechanosensitive microRNA-712 derived from pre-ribosomal RNA induces endothelial inflammation and atherosclerosis. Nat. Commun. 4:3000, 2013.

${ }^{193}$ Song, J. W., and M. S. Goligorsky. Perioperative implication of the endothelial glycocalyx. Korean J. Anesthesiol. 71(2):92-102, 2018.

${ }^{194}$ Song, J. W., J. A. Zullo, D. Liveris, M. Dragovich, X. F. Zhang, and M. S. Goligorsky. Therapeutic restoration of endothelial glycocalyx in sepsis. J. Pharmacol. Exp. Ther. 361(1):115-121, 2017.

${ }^{195}$ Starling, E. H. On the absorption of fluids from the connective tissue spaces. J. Physiol. 19(4):312-326, 1896. 
${ }^{196}$ Suwarto, S., R. T. Sasmono, R. Sinto, E. Ibrahim, and M. Suryamin. Association of endothelial glycocalyx and tight and adherens junctions with severity of plasma leakage in dengue infection. J. Infect. Dis. 215(6):992-999, 2017.

${ }^{197}$ Suzuki, K., and M. Terasawa. Biological activities of rhamnan sulfate extract from the green algae Monostroma nitidum (Hitoegusa). Mar. Drugs. 18(4):228, 2020.

${ }^{198}$ Tammi, R. H., A. Kultti, V. M. Kosma, R. Pirinen, P. Auvinen, and M. I. Tammi. Hyaluronan in human tumors: pathobiological and prognostic messages from cell-associated and stromal hyaluronan. Semin. Cancer Biol. 18(4):288-295, 2008.

${ }^{199}$ Tang, T. H., S. Alonso, L. F. Ng, T. L. Thein, V. J. Pang, Y. S. Leo, et al. Increased serum hyaluronic acid and heparan sulfate in dengue fever: association with plasma leakage and disease severity. Sci. Rep. 7:46191, 2017.

${ }^{200}$ Tarbell, J. M., and L. M. Cancel. The glycocalyx and its significance in human medicine. J. Intern. Med. 280(1):97113, 2016.

${ }^{201}$ Tarbell, J. M., and E. E. Ebong. The endothelial glycocalyx: a mechano-sensor and -transducer. Sci. Signal. 1(40):8, 2008

${ }^{202}$ Tarbell, J. M., and M. Y. Pahakis. Mechanotransduction and the glycocalyx. J. Intern. Med. 259(4):339-350, 2006.

${ }^{203}$ Targosz-Korecka, M., M. Jaglarz, K. E. Malek-Zietek, A. Gregorius, A. Zakrzewska, B. Sitek, et al. AFM-based detection of glycocalyx degradation and endothelial stiffening in the $\mathrm{db} / \mathrm{db}$ mouse model of diabetes. Sci. Rep. 7(1):15951, 2017.

${ }^{204}$ Targosz-Korecka, M., K. E. Malek-Zietek, D. Kloska, Z. Rajfur, E. L. Stepien, A. Grochot-Przeczek, et al. Metformin attenuates adhesion between cancer and endothelial cells in chronic hyperglycemia by recovery of the endothelial glycocalyx barrier. Biochim. Biophys. Acta Gen. Subj. 1864(4):129533, 2020.

${ }^{205}$ Thi, M. M., J. M. Tarbell, S. Weinbaum, and D. C. Spray. The role of the glycocalyx in reorganization of the actin cytoskeleton under fluid shear stress: a "bumper-car" model. Proc. Natl. Acad. Sci. USA. 101(47):16483-16488, 2004.

${ }^{206}$ Torres Filho, I. P., L. N. Torres, C. Salgado, and M. A. Dubick. Novel adjunct drugs reverse endothelial glycocalyx damage after hemorrhagic shock in rats. Shock. 48(5):583-589, 2017.

${ }^{207}$ Tuma, M., S. Canestrini, Z. Alwahab, and J. Marshall. Trauma and endothelial glycocalyx: the microcirculation helmet? Shock. 46(4):352-357, 2016.

${ }^{208}$ Tzima, E., M. Irani-Tehrani, W. B. Kiosses, E. Dejana, D. A. Schultz, B. Engelhardt, et al. A mechanosensory complex that mediates the endothelial cell response to fluid shear stress. Nature 437(7057):426-431, 2005.

${ }^{209}$ Uchimido, R., E. P. Schmidt, and N. I. Shapiro. The glycocalyx: a novel diagnostic and therapeutic target in sepsis. Crit. Care 23(1):16, 2019.

${ }^{210}$ Ueno, M., H. Sakamoto, Y. J. Liao, M. Onodera, C. L. Huang, H. Miyanaka, et al. Blood-brain barrier disruption in the hypothalamus of young adult spontaneously hypertensive rats. Histochem. Cell Biol. 122(2):131-137, 2004.

${ }^{211}$ Ueno, M., H. Sakamoto, H. Tomimoto, I. Akiguchi, M. Onodera, C. L. Huang, et al. Blood-brain barrier is impaired in the hippocampus of young adult spontaneously hypertensive rats. Acta Neuropathol. 107(6):532-538, 2004.

${ }^{212}$ van den Berg, B. M., J. A. Spaan, T. M. Rolf, and H. Vink. Atherogenic region and diet diminish glycocalyx dimension and increase intima-to-media ratios at murine carotid artery bifurcation. Am. J. Physiol. Heart. Circ. Physiol. 290(2):H915-H920, 2006.

${ }^{213}$ van den Berg, B. M., J. A. Spaan, and H. Vink. Impaired glycocalyx barrier properties contribute to enhanced intimal low-density lipoprotein accumulation at the carotid artery bifurcation in mice. Pflugers Arch. 457(6):11991206, 2009.

${ }^{214}$ van Haare, J., M. E. Kooi, J. W. van Teeffelen, H. Vink, J. Slenter, H. Cobelens, et al. Metformin and sulodexide restore cardiac microvascular perfusion capacity in dietinduced obese rats. Cardiovasc. Diabetol. 16(1):47, 2017.

${ }^{215}$ Van Wyngene, L., J. Vandewalle, and C. Libert. Reprogramming of basic metabolic pathways in microbial sepsis: therapeutic targets at last. EMBO Mol. Med. 10(8):7812, 2018.

${ }^{216}$ Vlahu, C. A., B. A. Lemkes, D. G. Struijk, M. G. Koopman, R. T. Krediet, and H. Vink. Damage of the endothelial glycocalyx in dialysis patients. J. Am. Soc. Nephrol. 23(11):1900-1908, 2012.

${ }^{217}$ Wagenseil, J. E., and R. P. Mecham. Elastin in large artery stiffness and hypertension. J. Cardiovasc. Transl. Res. 5(3):264-273, 2012.

${ }^{218}$ Wang, S., Y. Qiu, and B. Bai. The expression, regulation, and biomarker potential of glypican-1 in cancer. Front. Oncol. 9:614, 2019.

${ }^{219}$ Weinbaum, S. 1997 Whitaker distinguished lecture: models to solve mysteries in biomechanics at the cellular level; a new view of fiber matrix layers. Ann. Biomed. Eng. 26(4):627-643, 1998.

${ }^{220}$ Weinbaum, S., J. M. Tarbell, and E. R. Damiano. The structure and function of the endothelial glycocalyx layer. Annu. Rev. Biomed. Eng. 9:121-167, 2007.

${ }^{221}$ Weinbaum, S., X. Zhang, Y. Han, H. Vink, and S. C. Cowin. Mechanotransduction and flow across the endothelial glycocalyx. Proc. Natl. Acad. Sci. USA. 100(13):7988-7995, 2003.

${ }^{222}$ Weissgerber, T. L., O. Garcia-Valencia, N. M. Milic, E. Codsi, H. Cubro, M. C. Nath, et al. Early onset preeclampsia is associated with glycocalyx degradation and reduced microvascular perfusion. J. Am. Heart Assoc. 8(4):e010647, 2019.

${ }^{223}$ Wodicka, J. R., A. M. Chambers, G. S. Sangha, C. J. Goergen, and A. Panitch. Development of a glycosaminoglycan derived, selectin targeting anti-adhesive coating to treat endothelial cell dysfunction. Pharmaceuticals (Basel). 10(2):36, 2017.

${ }^{224}$ Woods, E. C., F. Kai, J. M. Barnes, K. Pedram, M. W. Pickup, M. J. Hollander, et al. A bulky glycocalyx fosters metastasis formation by promoting G1 cell cycle progression. Elife. 6:e25752, 2017.

${ }^{225} \mathrm{Xia}, \mathrm{Y}$., and B. M. Fu. Investigation of endothelial surface glycocalyx components and ultrastructure by single molecule localization microscopy: stochastic optical reconstruction microscopy (STORM). Yale J. Biol. Med. 91(3):257-266, 2018

${ }^{226}$ Xu, S., C. H. Ha, W. Wang, X. Xu, M. Yin, F. Q. Jin, et al. PECAM1 regulates flow-mediated Gab1 tyrosine phosphorylation and signaling. Cell Signal. 28(3):117-124, 2016.

${ }^{227}$ Yang, X., J. E. Meegan, M. Jannaway, D. C. Coleman, and S. Y. Yuan. A disintegrin and metalloproteinase 15mediated glycocalyx shedding contributes to vascular leakage during inflammation. Cardiovasc. Res. 114(13):1752-1763, 2018. 
${ }^{228}$ Yang, Y., Y. Zhao, J. Lan, Y. Kang, T. Zhang, Y. Ding, et al. Reduction-sensitive CD44 receptor-targeted hyaluronic acid derivative micelles for doxorubicin delivery. Int. J. Nanomed. 13:4361-4378, 2018.

${ }^{229}$ Yao, Y., A. Rabodzey, and C. F. Dewey, Jr. Glycocalyx modulates the motility and proliferative response of vascular endothelium to fluid shear stress. Am. J. Physiol. Heart Circ. Physiol. 293(2):H1023-H1030, 2007.

${ }^{230}$ Yao, M., L. Wang, M. Fang, W. Zheng, Z. Dong, and D. Yao. Advances in the study of oncofetal antigen glypican3 expression in HBV-related hepatocellular carcinoma. Biosci. Trends. 10(5):337-343, 2016.

${ }^{231}$ Yen, W., B. Cai, J. Yang, L. Zhang, M. Zeng, J. M. Tarbell, et al. Endothelial surface glycocalyx can regulate flow-induced nitric oxide production in microvessels in vivo. PLoS ONE 10(1):e0117133, 2015.

${ }^{232}$ Yen, W. Y., B. Cai, M. Zeng, J. M. Tarbell, and B. M. Fu. Quantification of the endothelial surface glycocalyx on rat and mouse blood vessels. Microvasc. Res. 83(3):337-346, 2012.

${ }^{233}$ Yeo, T. W., J. B. Weinberg, D. A. Lampah, E. Kenangalem, P. Bush, Y. Chen, et al. Glycocalyx breakdown is associated with severe disease and fatal outcome in Plasmodium falciparum malaria. Clin. Infect. Dis. 69(10):17121720, 2019.

${ }^{234}$ Yurdagul, Jr, A., A. C. Finney, M. D. Woolard, and A. W. Orr. The arterial microenvironment: the where and why of atherosclerosis. Biochem. J. 473(10):1281-1295, 2016.

${ }^{235}$ Zeng, Y., R. H. Adamson, F. R. Curry, and J. M. Tarbell. Sphingosine-1-phosphate protects endothelial glycocalyx by inhibiting syndecan-1 shedding. Am. J. Physiol. Heart. Circ. Physiol. 306(3):H363-H372, 2014.

${ }^{236}$ Zeng, Y., E. E. Ebong, B. M. Fu, and J. M. Tarbell. The structural stability of the endothelial glycocalyx after enzymatic removal of glycosaminoglycans. PLOS ONE 7(8):e43168, 2012.

${ }^{237}$ Zeng, Y., and J. Liu. Role of glypican-1 in endothelial NOS activation under various steady shear stress magnitudes. Exp. Cell Res. 348(2):184-189, 2016.

${ }^{238}$ Zeng, Y., X. H. Liu, J. Tarbell, and B. Fu. Sphingosine 1phosphate induced synthesis of glycocalyx on endothelial cells. Exp. Cell Res. 339(1):90-95, 2015.

${ }^{239}$ Zeng, Y., M. Waters, A. Andrews, P. Honarmandi, E. Ebong, V. Rizzo, et al. Fluid shear stress induces the clustering of heparan sulfate via mobility of glypican-1 in lipid rafts. Am. J. Physiol. Heart Circ. Physiol. 305:811820, 2013.

${ }^{240}$ Zeng, Y., X. F. Zhang, B. M. Fu, and J. M. Tarbell. The role of endothelial surface glycocalyx in mechanosensing and transduction. Adv. Exp. Med. Biol. 1097:1-27, 2018.

${ }^{241}$ Zhang, X., R. H. Adamson, F. R. Curry, and S. Weinbaum. A 1-D model to explore the effects of tissue loading and tissue concentration gradients in the revised Starling principle. Am. J. Physiol. Heart Circ. Physiol. 291(6):H2950-H2964, 2006.

${ }^{242}$ Zhang, X., R. H. Adamson, F. E. Curry, and S. Weinbaum. Transient regulation of transport by pericytes in venular microvessels via trapped microdomains. Proc. Natl. Acad. Sci. U S A. 105(4):1374-1379, 2008.

${ }^{243}$ Zhang, X., D. Sun, J. W. Song, J. Zullo, M. Lipphardt, L. Coneh-Gould, et al. Endothelial cell dysfunction and glycocalyx: a vicious circle. Matrix Biol. 71-72:421-431, 2018.

${ }^{244}$ Zhang, L., M. Zeng, J. Fan, J. M. Tarbell, F. R. Curry, and B. M. Fu. Sphingosine-1-phosphate maintains normal vascular permeability by preserving endothelial surface glycocalyx in intact microvessels. Microcirculation. 23(4):301-310, 2016.

${ }^{245}$ Zhang, L., M. Zeng, and B. M. Fu. Sphingosine-1-phosphate reduces adhesion of malignant mammary tumor cells MDA-MB-231 to microvessel walls by protecting endothelial surface glycocalyx. Cell Mol. Biol. 63(4):16-22, 2017.

${ }^{246}$ Zhong, Y., K. Goltsche, L. Cheng, F. Xie, F. Meng, C. Deng, et al. Hyaluronic acid-shelled acid-activatable paclitaxel prodrug micelles effectively target and treat CD44overexpressing human breast tumor xenografts in vivo. Biomaterials 84:250-261, 2016.

${ }^{247}$ Zhou, J., Y. S. Li, and S. Chien. Shear stress-initiated signaling and its regulation of endothelial function. Arterioscler. Thromb. Vasc. Biol. 34(10):2191-2198, 2014.

${ }^{248}$ Zullo, J. A., J. Fan, T. T. Azar, W. Yen, M. Zeng, J. Chen, et al. Exocytosis of endothelial lysosome-related organelles hair-triggers a patchy loss of glycocalyx at the onset of sepsis. Am. J. Pathol. 186(2):248-258, 2016.

Publisher's Note Springer Nature remains neutral with regard to jurisdictional claims in published maps and institutional affiliations. 